\title{
WestVirginiaUniversity
}

THE RESEARCH REPOSITORY @ WVU

Graduate Theses, Dissertations, and Problem Reports

2003

\section{Economics of breast cancer preventive strategies in a Medicaid program}

Rohit D. Borker

West Virginia University

Follow this and additional works at: https://researchrepository.wvu.edu/etd

\section{Recommended Citation}

Borker, Rohit D., "Economics of breast cancer preventive strategies in a Medicaid program" (2003).

Graduate Theses, Dissertations, and Problem Reports. 1842.

https://researchrepository.wvu.edu/etd/1842

This Dissertation is protected by copyright and/or related rights. It has been brought to you by the The Research Repository @ WVU with permission from the rights-holder(s). You are free to use this Dissertation in any way that is permitted by the copyright and related rights legislation that applies to your use. For other uses you must obtain permission from the rights-holder(s) directly, unless additional rights are indicated by a Creative Commons license in the record and/ or on the work itself. This Dissertation has been accepted for inclusion in WVU Graduate Theses, Dissertations, and Problem Reports collection by an authorized administrator of The Research Repository @ WVU.

For more information, please contact researchrepository@mail.wvu.edu. 


\title{
Economics of Breast Cancer Preventive Strategies in a Medicaid Program
}

\author{
Rohit D. Borker \\ Dissertation submitted to the \\ School of Pharmacy at West Virginia University \\ in partial fulfillment of the requirements \\ for the degree of \\ Doctor of Philosophy \\ in \\ Pharmaceutical Sciences \\ Suresh Madhavan, Ph.D., MBA, Chair \\ Gerald Higa, Pharm. D., BCOP \\ Mayur Amonkar, Ph.D. \\ Kenneth Simon, Ed.D. \\ Virginia Scott, Ph.D. \\ Department of Pharmaceutical Systems \& Policy \\ Morgantown, West Virginia \\ 2003
}

Keywords: Economic evaluation, Chemoprevention, Breast cancer Copyright 2003 Rohit D. Borker 


\begin{abstract}
Economics of Breast Cancer Preventive Strategies in a Medicaid Program
\end{abstract}

\author{
Rohit D. Borker
}

The primary objective of the study was to estimate the long-term benefits and costs of chemopreventive tamoxifen and mammography screening in women who are otherwise healthy but at high risk of developing breast cancer. Three hypothetical cohorts of highrisk women were initiated at age forty. The first cohort consumed chemopreventive tamoxifen and underwent routine mammography screening. The second cohort also consumed chemopreventive tamoxifen but underwent mammography screening at realworld rates. The third cohort (control cohort) did not consume chemopreventive tamoxifen and underwent mammography screening at real-world rates. The study also assessed the proportion of women in the West Virginia Medicaid Program (WVMP) who were healthy but at a high risk of developing breast cancer. Secondary objectives of the study involved determining breast cancer knowledge, utilization of breast cancer preventive strategies, and willingness to consume chemopreventive tamoxifen.

Chemopreventive tamoxifen coupled with routine mammography screening resulted in an incremental life expectancy gain of 0.122 years at an incremental cost of $\$ 5,969.70$ resulting in an incremental cost effectiveness ratio (ICER) of $\$ 48,931.80$ per life year gained (3\% discount rate) as compared to control cohort. Chemopreventive tamoxifen coupled with mammography screening at real-world rates resulted in an incremental life expectancy gain of 0.076 years at an incremental cost of $\$ 4,916.84$ resulting in an incremental cost effectiveness ratio (ICER) of $\$ 64,695.20$ per life year gained ( $3 \%$ discount rate) as compared to control cohort. Sensitivity analysis indicated robustness of results over a wide rage of assumptions. About half of the surveyed population was at a high risk of developing breast cancer and thus eligible to receive chemopreventive tamoxifen. However, only about $17 \%$ respondents indicated their inclination towards consuming chemopreventive tamoxifen. A low but significant correlation was observed between the respondent's perceived risk and real risk indicating the need for increasing breast cancer awareness. Respondent's actual risk of developing breast cancer did not appear to have any relationship with willingness to consume chemopreventive tamoxifen. In summary, chemopreventive tamoxifen plus mammography screening in high-risk women is a cost-effective strategy. However, at present, very few of these high-risk women may actually utilize chemoprevention. 


\title{
DEDICATION
}

This research is dedicated to

My Parents

DAMODAR AND SUNITA BORKER

\author{
My Wife \\ Yogita Bahl
}

My Brother-in-law and my Sister

Chetan and Dipti Desai 


\section{ACKNOWLEDGEMENTS}

First and foremost, I would like to thank my advisor and Committee Chairperson Dr. Suresh Madhavan for his advice, encouragement, and constant support throughout the course of this research. It is difficult for me to express my gratitude and respect for a researcher and a mentor of his caliber, and above all for such a fine human being. He has been a constant source of motivation and inspiration for me, especially during difficult phases of my dissertation. I owe a great deal of my success to him.

I would also like to thank my committee members, Dr. Mayur Amonkar, Dr. Gerald Higa, Dr. Kenneth Simon, and Dr. Virginia Scott, for their involvement and dedicated support throughout my graduate research. Their constructive criticism and comments/feedback on my research work enabled me to strengthen my study design and make the study more comprehensive and applicable. Also, I would like to extend my deepest appreciation and gratitude to Ms. Peggy King for her support and feedback on the 'survey research' phase of my dissertation.

I would also like to thank the staff at the School of Pharmacy, especially Debbie Anderson and Marion McGervey, for their help and support at times when I needed it most. I would also like to thank Tammy Richards, who worked with me when I was a research assistant, for being such a great colleague and friend. She is one of the most helpful person that I have ever met.

I would also like to thank all the graduate students for helping me out with survey mailing, especially Khalid and Aashish. Without their help I would still be sending out surveys instead of writing this section. I would also like to thank all my friends in Morgantown for making my graduate study experience a memorable one. I would especially like to thank Iftekhar Kalsekar, Khalid Kamal, Aashish Bhatia, Ancilla Fernandes, and Aashish Joshi for putting up with me for four good years. Thank you very much guys for your constant support and friendship.

I would like to thank my wife for her constant support and encouragement, especially during stressful times. Thank you Yogita for willingly putting up with me and my tantrums. 
I would also like to thank my brother-in-law and my sister without whose support I wouldn't be here. Thank you very much for believing in me and helping me to realize my dreams. I cannot thank you enough for all that you have done for me.

Finally and above all, I would like to thank my parents for everything that they have done for me, not just during my graduate career but my lifetime. I don't believe there are any words that can express my feelings and appreciation for what they have done and sacrificed just to get me to where I am today. Thank you very much for always believing in me and supporting me in my success and more importantly in my failures. I can never re-pay all that you have done for me. I dedicate this dissertation to you and hope that I have made you proud. 


\section{TABLE OF CONTENTS}

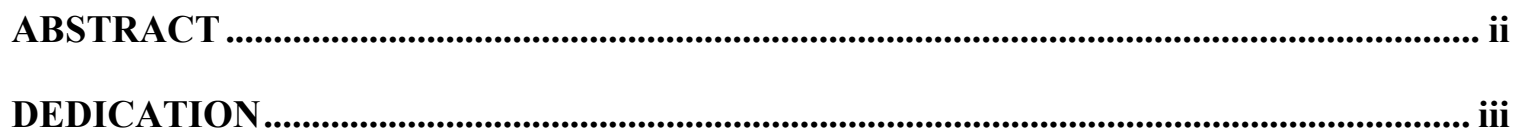

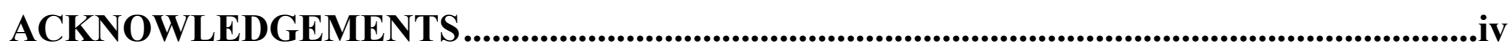

TABLE OF CONTENTS..................................................................................................................vi

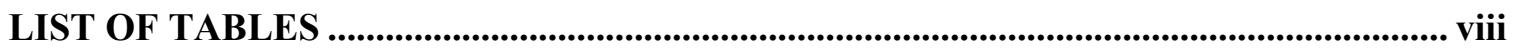

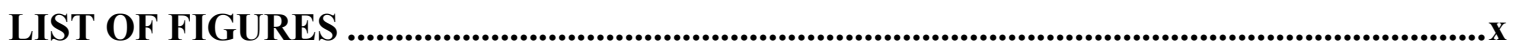

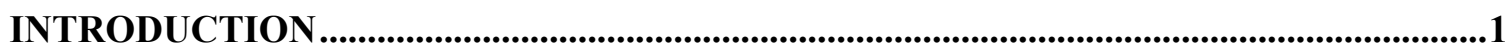

Epidemiology and Cost of Breast Cancer .........................................................................1

Risk factors for Breast Cancer...................................................................................................................2

Breast Cancer Prevention Strategies............................................................................................2

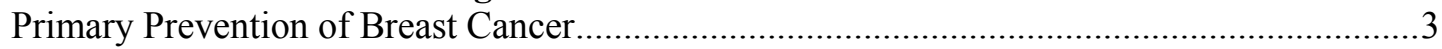

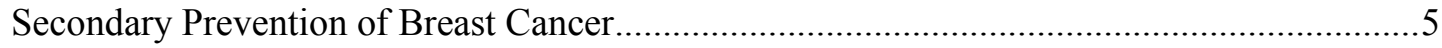

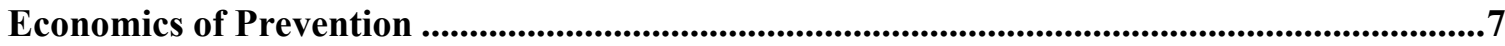

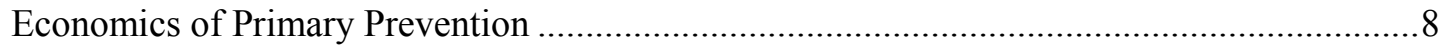

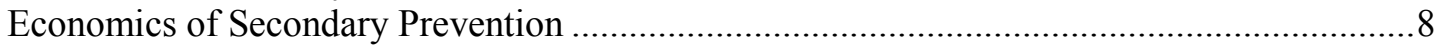

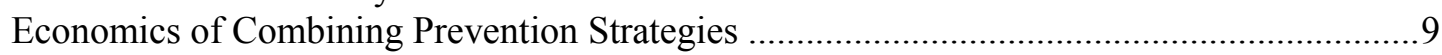

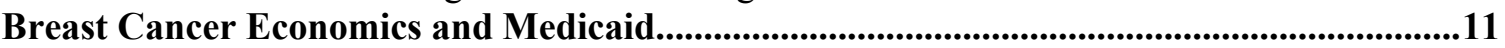

Rationale for selecting the West Virginia Medicaid Program ................................................11

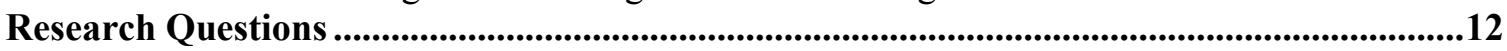

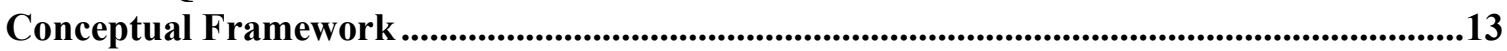

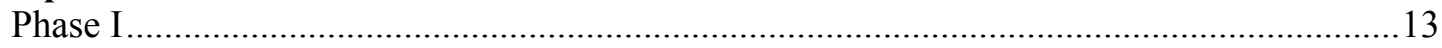

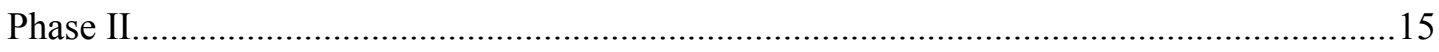

Study Goals and Objectives..........................................................................................................17

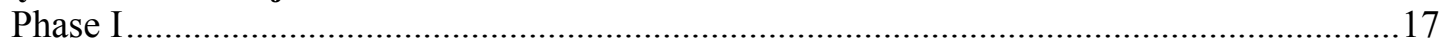

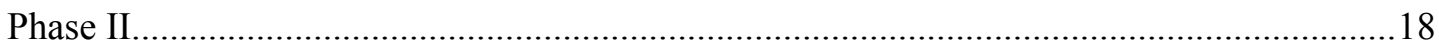

Significance of Research ...............................................................................................................20

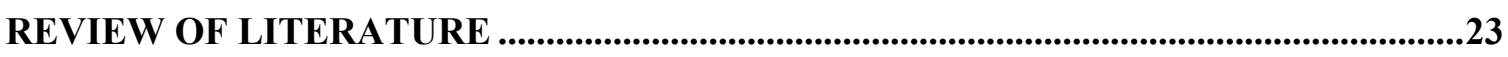

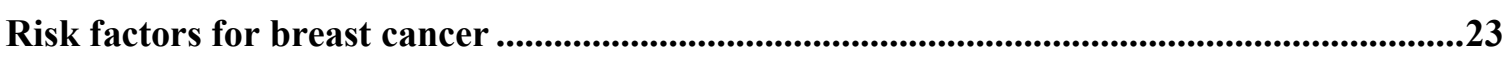

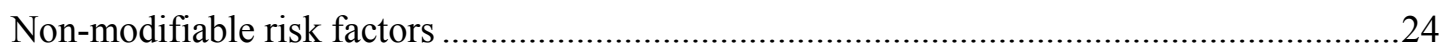

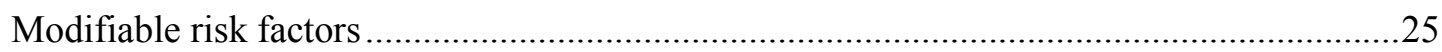

Models for assessing individual risk for breast cancer ........................................................26

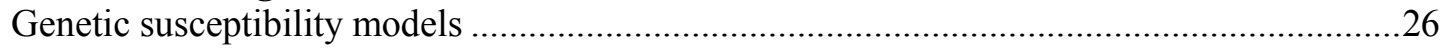

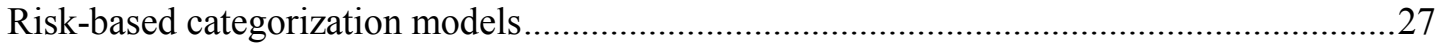

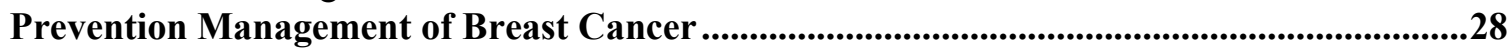

Evidence for Clinical Efficacy of Primary Prevention Strategy ……....................................28

Evidence for Clinical Efficacy of Secondary Prevention Strategy ..........................................31

Economics of Prevention Strategies.................................................................................................33

Economic Evaluation of Primary Prevention Strategy ............................................................35 
Economic Evaluation of Secondary Prevention Strategy ……................................................37

Perceptions of Breast Cancer Risk, Knowledge, and Preventive Service Utilization .............39

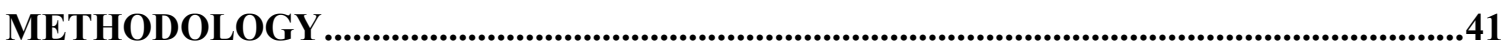

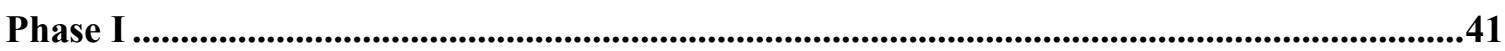

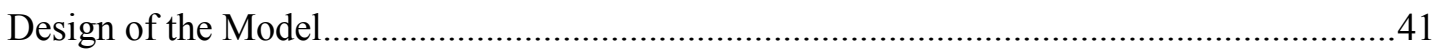

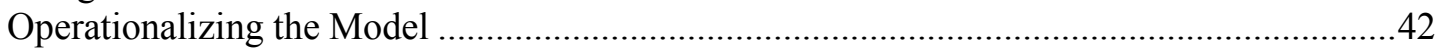

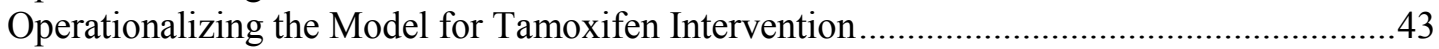

Operationalizing the Model for Mammography Screening Intervention................................44

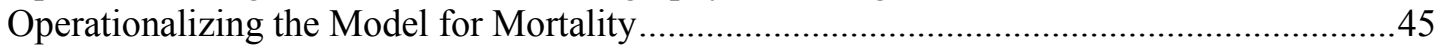

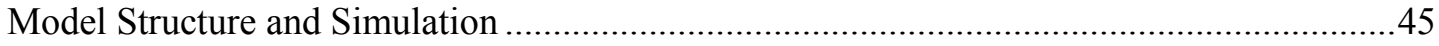

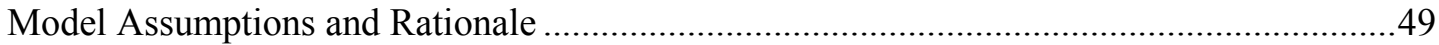

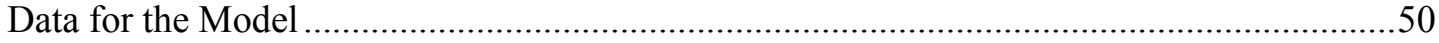

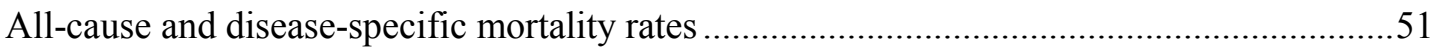

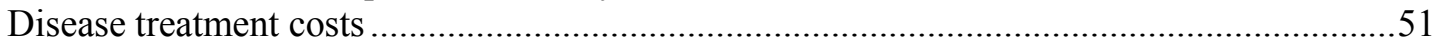

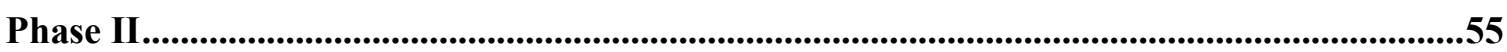

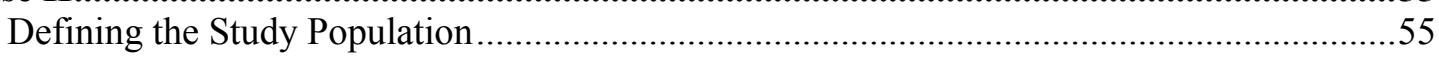

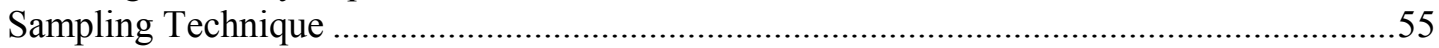

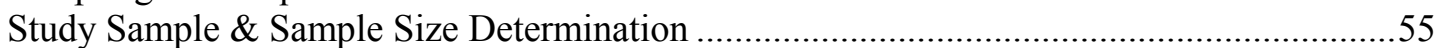

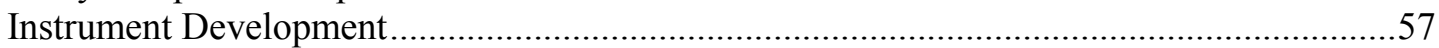

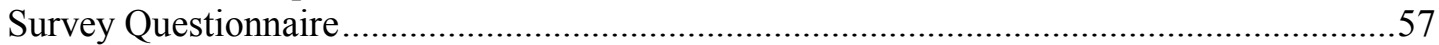

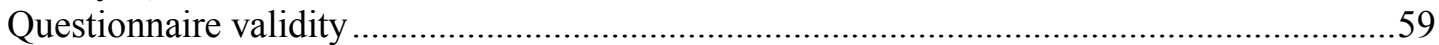

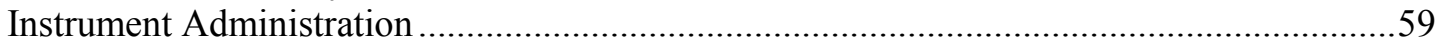

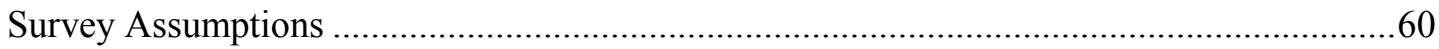

RESULTS............................................................................................................................................61

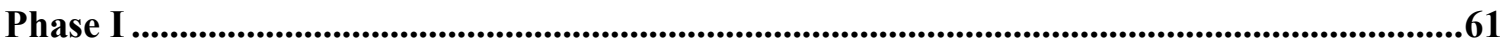

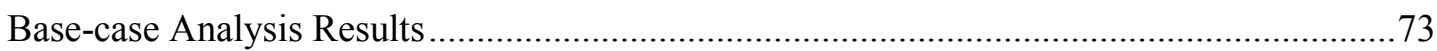

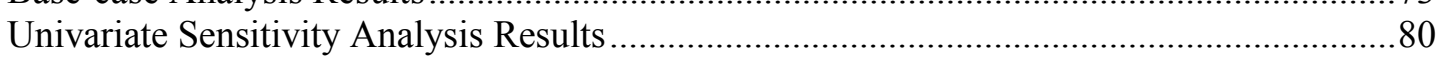

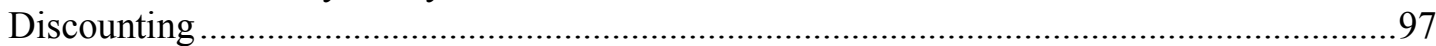

Tamoxifen in hysterectomized women (sub-group analysis) ...........................................102

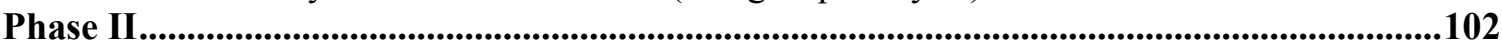

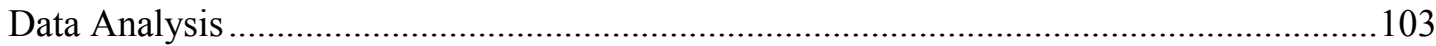

Relationships between actual risk of breast cancer, perceived risk of breast cancer, breast cancer knowledge, and screening behavior.........................................................................111

Relationships between perceived risk of breast cancer, breast cancer knowledge, and screening behavior .......................................................................................................112

Using chemopreventive tamoxifen and its relation to actual risk, and perceived risk ..........113

DISCUSSION \& RECOMMENDATIONS .................................................................................115

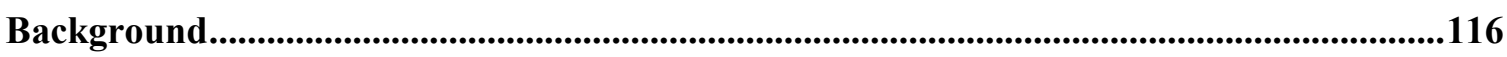

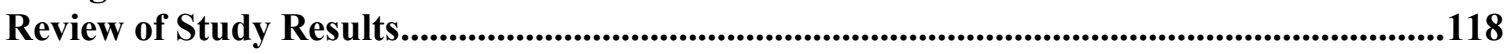

Implication of study findings.................................................................................................................124

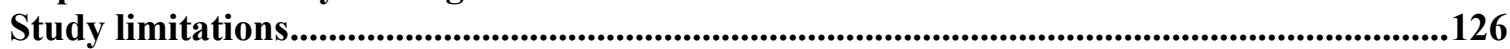

Recommendations for Future Research..................................................................................128

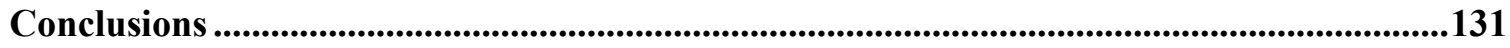

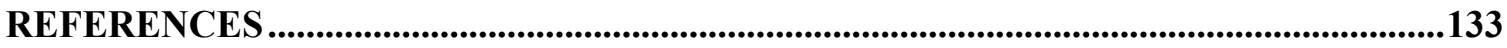

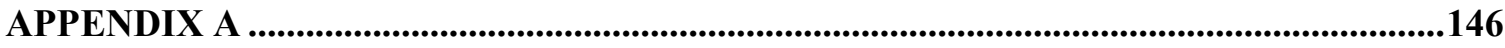

RESUME 


\section{LIST OF TABLES}

Table 3.1: Incidence Rates based on the BCPT study

Table 4.1: Transition Probability Matrix

Table 4.2: Age-dependent probabilities [H-Tam to other health states] .64

Table 4.3: Age-dependent probabilities [H-noTam to other health states] 66

Table 4.4: Invasive Breast Cancer (IBC)-adjusted mortality rates .68

Table 4.5: Non-Invasive Breast Cancer (NIBC)-adjusted mortality rates 69

Table 4.6: Endometrial Cancer (EC)-adjusted mortality rates ..............................70

Table 4.7: Pulmonary Embolism (PE)-adjusted mortality rates .............................71

Table 4.8: Deep Vein Thrombosis (DVT)-adjusted mortality rates .............................72

Table 4.9: Simulated Cohort Life-Years Distribution - Base-case ..............................74

Table 4.10: Simulated Cohort Cost Distribution - Base-case ...............................76

Table 4.11: Incremental Cost-effectiveness Ratios for the Cohorts ..........................79

Table 4.12: One-way Sensitivity Analysis at $3 \%$ Discount Rate:

Efficacy Parameters

Table 4.13: One-way Sensitivity Analysis at $3 \%$ Discount Rate:

Efficacy Parameters and Incremental Analysis

Table 4.14: One-way Sensitivity Analysis at $3 \%$ Discount Rate:

Cost Parameters for IBC and NIBC

Table 4.15: One-way Sensitivity Analysis at 3 \% Discount Rate:

Cost Parameters and Incremental Analysis for IBC and NIBC

Table 4.16: One-way Sensitivity Analysis at $3 \%$ Discount Rate:

Cost Parameters for Non-breast Cancer States

Table 4.17: One-way Sensitivity Analysis at 3 \% Discount Rate: Cost

Parameters and Incremental Analysis for Non-breast Cancer States

Table 4.18: One-way Sensitivity Analysis at $3 \%$ Discount Rate:

Cost Parameters for Intervention Strategies 
Table 4.19: One-way Sensitivity Analysis at 3 \% Discount Rate: Cost

Parameters and Incremental Analysis for Intervention Strategies

Table 4.20: One-way Sensitivity Analysis at $5 \%$ Discount Rate:

Efficacy Parameters ......................................................98

Table 4.21: One-way Sensitivity Analysis at $5 \%$ Discount Rate:

Efficacy Parameters and Incremental Analysis ....

Table 4.22: One-way Sensitivity Analysis at 0 \% Discount Rate:

Efficacy Parameters

Table 4.23: One-way Sensitivity Analysis at 0 \% Discount Rate:

Efficacy Parameters and Incremental Analysis

Table 4.24: Characteristics of All Respondents .......................................104

Table 4.25: Proportion of women correctly responding to knowledge items .................106

Table 4.26: Characteristics of Respondents at High Risk for Breast Cancer ...............108

Table 4.27: Proportion of high-risk women who gave correct responses on knowledge items 110

Table 4.28: Knowledge Score and Respondent's Actual Risk of Breast Cancer .............112

Table 4.29: Knowledge Score and Respondent's Perceived Risk of Breast Cancer .........113

Table 4.30: Willingness to consume chemopreventive tamoxifen and breast cancer risk 


\section{LIST OF FIGURES}

Figure 3.1: The Transition State Diagram ............................................46

Figure 4.1: Simulated Cohort Life-Years Distribution for

Combined States- Base-case ...............................................75

Figure 4.2: Simulated Cohort Cost Distribution - Base-case ................................77

Figure 4.3: Sensitivity Analysis: Tam + Optimal Mam Screen vs.

NoTam+Non-optimal Mam Screen

Figure 4.4: Sensitivity Analysis: Tam + Non-optimal Mam Screen vs.

NoTam + Non-optimal Mam Screen ........................................84

Figure 4.5: Sensitivity Analysis: Tam + Optimal Mam Screen vs. Tam +

Non-optimal Mam Screen

Figure 4.6: Sensitivity Analysis: Tam + Optimal Mam Screen vs.

NoTam + Non-optimal Mam Screen

Figure 4.7: Sensitivity Analysis: Tam + Non-optimal Mam Screen vs.

NoTam + Non-optimal Mam Screen . .95

Figure 4.8: Sensitivity Analysis: Tam + Optimal Mam Screen vs.

Tam + Non-optimal Mam Screen .96 


\section{CHAPTER 1}

\section{INTRODUCTION}

\section{Epidemiology and Cost of Breast Cancer}

Breast cancer is the development of malignant tumor from the cells of the breast. Although breast cancer is one of the best studied human tumors, it still remains poorly understood. As a result, $30 \%$ to $40 \%$ of women who develop breast cancer die from it (Kopans, 1998). It is one of the most common cancers ranking second only to lung cancer in terms of cancer attributed deaths in women. In fact, breast cancer remains the leading cause of non-preventable cancer deaths in American women (Kopans, 1998). The American Cancer Society (ACS) estimates about 40,200 breast cancer deaths in the United States in 2001 (American Cancer Society, 2001-2002). Furthermore, approximately 1 in every 8 women will develop breast cancer by age of 85 years (American Cancer Society, 2001-2002) and about 192,200 new cases of invasive breast cancer will be diagnosed among women in the United States in 2001 (American Cancer Society, 2001-2002).

The breast cancer incidence over the years can be categorized into three distinct phases: (1) between 1940 and 1982, there was a steady rate of increase of about $1 \%$ annually (Garfinkel, 1994), (b) between 1982 and 1987, the increase was about $4 \%$ annually (Garfinkel, 1994), and (c) between 1990 and 1994, the incidence of developing breast cancer stabilized at about 110.2 cases per 100,000 women (Ries et al, 1997). In addition to these invasive cases, about 39,900 new cases of ductal carcinoma in-situ (DCIS) (Stage 0) are diagnosed each year.

Carcinoma in situ refers to non-invasive type of breast cancers in which the cancer cells inside the ducts do not spread through the walls of the ducts into fatty tissue of the breast. Between 1983 to 1993, in situ breast cancer rates have increased from 2.3 to 6.2 per 100,000 among women under age of 50 , and from 14.3 to 54.6 per 100,000 
among women aged 50 and older (Ries et al, 1997). As a result, breast cancer has been identified as a major public health problem (Serxner, Chung, 1992).

Breast cancer also entails substantial economic burden, both for an individual as well as for the society. For instance, the National Institutes of Health (NIH) estimates that a significant proportion of direct medical costs associated with cancer are due to breast cancers ( $\$ 6$ billion), lung cancers ( $\$ 5$ billion), and prostate cancers ( $\$ 5$ billion) (1990 US Dollars) (American Cancer Society, 2002). Furthermore, lifetime cost of breast cancer has been estimated to be about $\$ 36,926$ per patient (1984 dollars) (Baker et al., 1991). This translates to about $\$ 94,000$ per patient when inflated to 2001 dollars (assuming all other factors remain constant) based on Medical Care component of Consumer Price Index (CPI) (U.S. Bureau of Labor Statistics). This considerable economic burden coupled with clinical significance has prompted interest in breast cancer preventive strategies that are not only effective, but also economically viable.

\section{$\underline{\text { Risk factors for Breast Cancer }}$}

Risk of breast cancer increases with increasing age. Other factors that increase breast cancer risk are family history of breast cancer, biopsy confirmed atypical hyperplasia, a long menstrual history (menstrual period that starts early in life and ends late), and nulliparity or first live birth after age 30. Moreover, dietary and lifestyle behaviors such as alcohol intake, fat intake, recent consumption of oral contraceptives or post menopausal estrogen, weight gain and physical inactivity, among others, have also been linked to breast cancer risk although the causal role for some of these factors is still to be established. Each of these risk factors is discussed in more details in Chapter 2.

\section{Breast Cancer Prevention Strategies}

Currently no strategy exists to prevent breast cancer. Hence, the focus is on either reducing the risk of incident breast cancer or on detection of the already developed cancers at an earlier stage, thereby reducing mortality from it (Bush et al., 1993; Shapiro et al., 1982; Tabar et al., 1985). Strong evidence suggests that early detection has reduced breast cancer mortality in some groups of women (Tabar et al., 1985; Tabar et 
al., 1995; Kerlikowske et al., 1995; Hendrick et al., 1997). Despite the effectiveness of detecting breast tumors early, the sheer number of women affected by breast cancer has prompted efforts to control the disease using primary prevention strategies which involves reducing the incidence of breast cancer (National Cancer Institute, 2003). Both the primary and the secondary breast cancer preventive strategies are discussed next.

\section{Primary Prevention of Breast Cancer}

The rationale for primary prevention of breast cancer was based on wide variation in breast cancer rates across the world suggesting that environmental and lifestyle determinants were linked to breast cancer. For instance, it was reported that Japanese migrants to the United States acquired a significant portion of breast cancer risk of the host country within two generations (National Cancer Institute, 2003). Subsequently, studies have been conducted to examine the effects of environmental, dietary, and lifestyle factors on breast cancer risk (National Cancer Institute 2003; Wolff et al., 1993; Shames et al., 1994; Krieger et al., 1994; Hunter et al., 1997; Hunter et al., 1996). The results of these studies have been inconsistent and hence evidence linking these factors to the risk of breast cancer is weak. Lack of evidence coupled with inadequate understanding of the biologic mechanisms through which the risk factors interact has further limited the potential for primary prevention of breast cancer (Bush T et al., 1993). Nevertheless, a number of potential strategies are currently being evaluated in primary prevention of breast cancer. The term 'prevention' in the present context of primary prevention refers to a reduction in incidence (risk) of breast cancer over a defined period of time. Thus, although a strategy might prevent the appearance of significant number of tumors in a population over a defined period of time, it does not imply that initiation of breast cancers has been prevented or that tumors have been permanently eliminated. Primary prevention strategies are discussed next.

\section{Prophylactic Mastectomy}

Non-pharmacologic primary prevention strategies such as prophylactic mastectomy have been investigated with some promising results. A study reported an 89 $\%$ breast cancer risk reduction in moderate-risk women and a 90 to94 \% risk reduction in 
high-risk women, who underwent mastectomy (Hartmann, Schaid, Woods, et al., 1999). The potential for widespread implementation of prophylactic mastectomy in breast cancer risk reduction is, however, limited as most women who will undergo mastectomy (a procedure which involves extensive and potentially disfiguring surgery that may psychologically affect a patient's long-term quality of life (Stegfanek et al., 1995) will not go on to develop breast cancer (National Cancer Institute, 2003). Moreover, a task force set up by the National Institutes of Health and the National Human Genome Research Institute concluded that there is not enough evidence to support or recommend either for or against prophylactic mastectomy (Burke et al., 1997).

\section{Chemoprevention}

Limited ability to modify breast cancer risk factors provided an impetus for investigating chemoprevention (Bush et al., 1993) as a primary preventive strategy. Research in carcinogenesis has shown that greater than $90 \%$ of cancers are associated with mutagens and mitogens (Kelloff et al., 1997(a); Kelloff et al., 1997(b)). As a result, current research in breast cancer has been directed towards searching for agents that inhibit or reverse cellular processes resulting from mutagensis and mitigenesis. This process is called chemoprevention and is defined as prevention of cancer using pharmacological agents that inhibit or reverse the process of carcinogenesis (Sporn et al., 1979). This approach has yielded agents, which fall under the broad categories of retinoids, and antiestrogens/ antiandrogens (Kelloff et al., 1999). The later category includes a class of therapeutic agents known as selective estrogen receptor modulators (SERMs) which has shown most promise in chemoprevention of breast cancer (National Cancer Institute, 2003). Tamoxifen, which is a SERM, is one such agent that reduced the risk of breast cancer in high-risk women in the National Surgical Adjuvant Breast and Bowel Project (NSABP) Breast Cancer Prevention Trial (BCPT) protocol P-1 study (Fisher et al., 1998).

Currently, tamoxifen appears to be the most promising agent in primary prevention of breast cancer. Also, it is the only United States Food and Drug Administration (US FDA) approved drug for reducing breast cancer risk in high-risk 
women (American Society of Clinical Oncology, 2002). Hence, for the purpose of this study, tamoxifen will be considered as a strategy of choice for primary prevention of breast cancer in women who are otherwise healthy but at high risk of developing the disease.

Promising as it may seem, the scope of primary prevention has been restricted to women at high risk of developing breast cancer because of inherent limitations of these strategies. Owing to failure of etiologic studies in breast cancer research to identify primary prevention strategies suitable for the general population, reducing mortality through early detection of breast cancer still remains the mainstay (National Cancer Institute).

\section{Secondary Prevention of Breast Cancer}

The ACS guidelines list clinical breast examination (CBE), breast selfexamination (BSE) and mammography as secondary prevention strategies for early detection of breast cancer (American Cancer Society, 2001-2002). Following is a brief description of each of these strategies.

\section{Clinical Breast Examination}

A recent pooled analysis study investigated the role of clinical breast examination (CBE) in early detection of breast cancer (Barton et al., 1999). The study reported a reduction in breast cancer mortality in a group of patients screened by both CBE and mammography. However, the study indicated that evidence pertaining to CBE's contribution in reducing the mortality was less direct and that precision of $\mathrm{CBE}$ was difficult to assess due to lack of consistent and standardized examination techniques. Based on the pooled analysis, the CBE sensitivity (ability to detect cancer when the woman being screened has the disease) was estimated at $54 \%$, while the specificity (the ability to correctly identify a woman being screened as not having breast cancer when she does not have the disease) was $94 \%$. 


\section{Breast Self-examination}

There have been mixed results regarding the efficacy of breast self examination (BSE) in reducing breast cancer related mortality. A study by Thomas et al., (1997) reported neither lower death rates nor a shift towards the diagnosis of less advanced disease among subjects practicing BSE. Results from non-randomized trials in United Kingdom have not been very encouraging either. For instance, the relative risk of death from breast cancer was 1.13 (95 \% CI: 0.95-1.35) for one BSE center and 0.78 (95\% CI: 0.61-1.00) in the other center (UK Trial of Early Detection of Breast Cancer Group, 1993). However, a Finnish cohort study by Gastrin et al (1994), followed a large number of women and reported significantly lower breast cancer mortality rates in the group who performed BSE in comparison to the group of women who did not practice BSE.

\section{Screening Mammography}

Mammography is the most effective method for breast cancer screening, because of its ability to detect cancers before physical symptoms become apparent (American Cancer Society, 1997). In fact, the primary reason to perform mammography screening is to detect the occult cancer at an early stage so that the natural history of the disease can be interrupted, subsequently, reducing number of deaths from breast cancer (Kopans, 1998). Mammograms has sensitivity of about $76 \%$ to $94 \%$, which is much higher then that of clinical breast examination which has sensitivity in the range of $57 \%$ to $70 \%$. Also, screening mammography's specificity is greater than $90 \%$ (American Cancer Society, 1997). Moreover, radiation exposure with mammography is in a range of about 0.1 to 0.2 rad dose per $\mathrm{x}$-ray, not significant enough to pose any radiation exposure problems (American Cancer Society, 1997).

A large number of trials have shown that mammography effectively detects breast cancer at early stages, consequently increasing survival and decreasing breast cancer mortality by at least 30 \% (Costanza, 1992; Shapiro, 1988; Shapiro, 1989; Seidman et al., 1987; Tabar et al., 1992). Controversy, however, surrounds with respect to the appropriate age at which to begin regular mammography screening (Ransohoff et al., 1997). A Health Insurance Plan study reported breast cancer mortality reduction 
attributable to mammography screening in women aged 50 to 64 years but failed to show similar results for women between 40 to 49 years of age (Shapiro, 1988). Based on series of randomized controlled trials, case-control trials and demonstration projects, the NIH Consensus Development Conference in 1997 concluded that "the available data do not warrant a single recommendation for mammography for all women in their forties. Each women should decide for herself whether to undergo mammography (NIH Consensus Statement, 1997)." The ACS however took a different stand based on the same evidence and recommended an annual mammography for all women in their forties (American Cancer Society's Workshop on Guidelines for Breast Cancer Detection, 1997).

In conclusion, regardless of differences in effectiveness of mammography screening across different age groups, it is well established that mammography is the single most effective secondary prevention strategy for breast cancer in comparison with other secondary prevention strategies (National Cancer Institute). Consequently, mammography will be considered as the strategy of choice for secondary prevention of breast cancer in present study.

\section{Economics of Prevention}

Rapidly rising costs of medical technologies and services is increasingly reducing the ability to give all patients the care that would benefit them (Brook et al., 1990). Consequently, it has become necessary to define and determine the appropriateness of care in such an increasingly cost-conscious environment (Brook et al., 1990). Issues pertaining to effectiveness of preventive services and economic utility have become critical. An important aspect of any preventive intervention in an environment of finite resources is whether there are economic benefits to help offset the additional cost of prevention.

The clinical effectiveness of both primary and secondary breast cancer preventive strategies has been well established as is evident from the above discussion. However, a decision to implement any such prevention strategies require evidence of not only their 
effectiveness but also the costs that are incurred due to their implementation (Wright, 1986).

\section{Economics of Primary Prevention}

Very few studies have addressed the cost-effectiveness of tamoxifen in primary prevention of breast cancer. A Medline search (1966-present) identified only three studies, which have scientifically evaluated economic efficiency of using tamoxifen in women who are otherwise healthy but at a high risk of developing breast cancer. A study by Noe L et al., (1999) used a Markov model to study the incremental cost-effectiveness of tamoxifen in primary prevention of breast cancer. Incremental cost-effectiveness of tamoxifen was found to be $\$ 41,372$ per life years gained for women age 35 to 49 years and $\$ 68,349$ and $\$ 74,981$ per life years gained for women in their fifties and sixties respectively.

In an another study, which also employed the Markov model, the incremental cost-effectiveness of tamoxifen in primary prevention of breast cancer was found to be $\$ 46,619$ per life years saved for women who started on tamoxifen therapy at the age of 35 years (Grann et al., 2000). A study by Smith et al (2000) analyzed a base-case of highrisk women regardless of age and estimated a significantly lower cost-effectiveness ratio for tamoxifen of $\$ 8,479$ per life year saved. A major limitation of the latter analysis was the assumption of lifetime effectiveness of tamoxifen in breast cancer prevention. These studies are discussed in more details in Chapter 2.

\section{$\underline{\text { Economics of Secondary Prevention }}$}

Mammography has been the single most effective method for early detection of breast cancer, since it can identify cancers several years before the cancer becomes clinically evident (American Cancer Society, 2001-2002). There have been many studies, which have evaluated the cost-effectiveness of mammography in secondary prevention of breast cancer (Salzmann et al., 1997; Lindfors et al., 1995; Feig, 1995). A study by Salzmann et al., (1997) calculated average cost-effectiveness by comparing 
screening among different age cohorts of women (40-49 years, 50-69 years) versus no screening. The study reported a cost-effectiveness ratio of $\$ 105,000$ per life years saved for women undergoing screening in their forties. For women above 50 years, the costeffectiveness ratio was better at $\$ 21,000$ per life year saved. However, the costeffectiveness ratio has shown wide variations across studies. For instance, a study by Rosenquist C et al., (1998) reported the marginal cost per life year saved from mammography screening to be as low as $\$ 16,100$ to $\$ 18,800$. Part of the differences in mortality reduction can be attributed to mammography screening and to failure on part of some economic studies to include time lag between actual initiation of mammography and time when benefits in terms of mortality reduction start accruing (Salzmann et al., 1997).

\section{$\underline{\text { Economics of Combining Prevention Strategies }}$}

Chemopreventive tamoxifen and mammography screening have different roles in breast cancer prevention management. Chemopreventive tamoxifen reduces the risk of breast cancer while mammography screening results in early detection of breast tumors thereby improving survival. Their unique mode of actions makes these two strategies complimentary rather than competitive. The rationale for investigating the economics of tamoxifen and mammography screening in combination, thus, is based on the reasoning that chemopreventive tamoxifen does not eliminate the need for mammography screening. In other words, women who are eligible to receive preventive tamoxifen therapy would need to undergo routine mammography screening irrespective of whether they are taking chemopreventive tamoxifen or not. Thus, a policy for introducing tamoxifen for prevention of breast cancer should also consider the simultaneous effects (both costs and effectiveness) of mammography screening. This presents policy makers with two main scenarios: (1) use chemopreventive tamoxifen in tandem with mammography screening or (2) continue using mammography screening alone. An incremental analysis of these two scenarios will quantify cost and benefits of introducing chemopreventive tamoxifen versus not using chemoprevention. 
The greatest reduction in breast cancer mortality will result from the most optimum utilization of both preventive strategies. Hence, the first intervention that was included in the model was chemopreventive tamoxifen with routine mammography screening in accordance with the ACS guidelines. However, the optimal utilization will also come at a substantial cost in terms of cost of tamoxifen therapy and screening mammography. Hence, it is important to assess whether these additional costs that result from optimal utilization justify the benefits that they deliver in terms of reduction in breast cancer mortality. As is usually the case in real world setting, optimal utilization of interventions is seldom observed which compromises the effectiveness of that intervention. This sub-optimal utilization may also affect the total costs of the intervention in terms of decreasing intervention costs (due to lower utilization of the intervention as compared to optimal setting) and increasing non-intervention costs (due to increased proportion of detected cancers in later stages than in earlier stages thereby increasing treatment cost, etc). Hence, a second intervention was added where by the women took chemopreventive tamoxifen but underwent mammography screening at rates that were observed in real world setting. The third intervention, which acted as the control intervention, depicted the 'current state of affair' wherein women did not consume chemopreventive tamoxifen but underwent mammography at rates observed in a real world setting. An incremental cost effectiveness analysis of these interventions will quantify and compare the costs and benefits of each of the three strategies enabling policy makers to choose the most efficient strategy among the three.

Previous attempts at quantifying these costs and benefits of chemopreventive tamoxifen and thereby determining its efficiency in third party payer setting assumed a somewhat narrow perspective by excluding costs and benefits of mammography screening, an essential and expensive component of breast cancer prevention management. Thus, although these studies did provide information on economic efficiency of tamoxifen therapy, they did not address the issue of overall economics of breast cancer prevention. 


\section{Breast Cancer Economics and Medicaid}

Although the cost-effectiveness model to be developed in the present study will be applicable in most third party payer settings, this study will determine the incremental cost-effectiveness of the different scenarios in the West Virginia Medicaid Program (WVMP).

\section{$\underline{\text { Rationale for selecting the West Virginia Medicaid Program }}$}

Medicaid is the primary source of health care for low-income families with children, the low-income elderly, and disabled persons. The Medicaid program provides coverage on a wide range of basic health and long-term care services for more than 34 million individuals (www.urban.org/news/factsheets/medicaidFS.html). Title XIX of the Social Security Act requires that in order to receive Federal matching funds, the state Medicaid program must cover certain basic services including inpatient hospital services, outpatient hospital services, physician services, medical and surgical services, laboratory and x-ray services and early and periodic screening, diagnosis, and treatment (EPSDT) services (www.hcfa.gov/medicaid/mservice.html).

On January 2001, the Congressional Budget Office (CBO) released baseline projections for Federal Medicaid spending. The CBO estimated that the rate of Medicaid expenditure growth will rise from $8.7 \%$ in Federal fiscal year 2000 to $10.6 \%$ in 2001. In addition to the $\mathrm{CBO}$, many state level projections also forecast a rise in the rate of growth of Medicaid expenditures.

Increases in outpatient prescription drug expenditures have been reported to be the primary reason for significant increases in state Medicaid expenditures. Overall, Medicaid prescription drugs expenditures rose $20 \%$ in 1998. Moreover, Centers for Medicare and Medicaid (CMS) projects that Medicaid prescription drug expenditures will rise about $70 \%$ faster than overall Medicaid expenditures between 2001 and 2006. Since other major federal programs including Medicare do not cover prescription drugs, the increasing cost and utilization of prescription drugs creates substantial financial burden on Medicaid Programs (The Urban Institute, www.urban.org/news/factsheets/medicaidFS.html). 
Under such circumstances of rising Medicaid prescription drug expenditures, additional costs of newer drugs or preventive indications for drugs such as tamoxifen may entail a significant financial burden on the system. These additional costs, however, may be offset in case of some drugs through their appropriate and effective use, which may reduce other medical costs through decrease in physician office, ER visits, and hospitalizations (The Urban Institute, www.urban.org/news/factsheets/medicaidFS.html).

According to the CMS (1998), out of 308,910 Medicaid enrollees in the WVMP, about $30 \%(90,360)$ were women. Some proportion of these women will be at high risk of developing breast cancer. Introducing tamoxifen as a preventive strategy will entail certain drug costs to the Medicaid program including costs associated with the treatment of adverse effects of tamoxifen. These costs will be over and above the cost of mammography screening, which the Medicaid program is already incurring. The Medicaid program will also experience some cost savings due to a reduction in the number of breast cancers through use of chemopreventive tamoxifen. A costeffectiveness analysis will thus be required to determine if beneficial effects of tamoxifen help offset its additional costs in presence of mammography screening.

The Medicaid program provides an appropriate female population to study the economic efficiency of tamoxifen and mammography screening. The results of the proposed study will determine whether or not tamoxifen as a chemopreventive agent and mammography screening as a secondary preventive technique will lead to cost-savings in the Medicaid population and help Medicaid reduce its overall expenditures. The following section details a list of research questions, the answers to which will help to understand the economics of breast cancer prevention in the Medicaid setting.

\section{$\underline{\text { Research Questions }}$}

Q1. What are the incremental costs and consequences of introducing chemopreventive tamoxifen in tandem with mammography screening in the West Virginia Medicaid Program (WVMP)? 
Q2. What proportion of women enrollees in the WVMP are at a high risk of developing breast cancer and thus eligible for chemopreventive tamoxifen intervention?

Q3. What proportion of women enrollees in the WVMP are willing to accept chemopreventive tamoxifen for reducing their risk of breast cancer?

Q4. What is the relationship between their actual risk and perceived risk of developing breast cancer, their knowledge of breast cancer and breast cancer preventive strategies and willingness to consume chemopreventive drugs?

\section{Conceptual Framework}

This study will be conducted in two phases. Phase I involves construction of an economic model to compare the incremental cost-effectiveness of breast cancer preventive strategies. Phase II will estimate the proportion of WVMP women population at high risk of developing breast cancer. Information on women's perceived risk of developing breast cancer and their knowledge about breast cancer preventive strategies will also be assessed in phase II.

\section{Phase I}

Phase I involves developing a decision analytic model to systematically compare different breast cancer preventive interventions discussed in the earlier section titled "Economics of Combining Prevention Strategies." In general, a health condition can be managed using different therapeutic options. These options may have different effects on the natural history of the disease. The challenge then is to quantify these effects and identify those therapies that deliver maximum benefits in the most efficient manner for a given health condition. Decision analysis process allows quantification of these effects methodically determining the costs and consequences of the intervention.

The current study will simulate three hypothetical cohorts of 40-year old women who are otherwise healthy but at a high risk of developing breast cancer. The first cohort assumes that all women will begin taking chemopreventive tamoxifen and will undergo routine mammography screening in accordance with the ACS recommended screening guidelines. Compliance with tamoxifen will however be adjusted for actual tamoxifen 
compliance rates as observed in the tamoxifen chemopreventive trial (Fisher et al., 1998). The second cohort represents a much more likely scenario wherein all women will begin taking chemopreventive tamoxifen and will undergo mammography screening at rates, which are observed in real-world setting. The third cohort represents the control cohort in that these women will not take chemopreventive tamoxifen and will undergo mammography screening at 'observed' rates (as observed in real-world setting), which will similar to the rates assumed in the second cohort but lower than the optimal mammography compliance assumed in the first cohort.

The present study involves extrapolating results from the short-term ( 5 year) BCPT and mammography trials over the patient's lifetime to quantify effects of introducing chemopreventive tamoxifen in addition to mammography screening. In such cases, Markov models have been found to be particularly useful and highly applicable due to their ability to intuitively handle both costs and outcomes simultaneously over chronic time intervals (Briggs A et al., 1998). Markov models have been regularly employed in medical decision making for many years (Sonnenberg et al., 1993). More on actual working of Markov models are presented in Chapter 2.

The decision to follow women at the age of forty was based on the rationale that only women in their forties and above have been recommended annual mammography screening by the ACS. Relatively low sensitivity of mammography (Hicks et al., 1979) accompanied by relatively low breast cancer incidence rates (Harris et al., 1991) makes routine mammography screening very inefficient in women below 40 years of age.

The primary outcomes of Phase I are incremental life expectancy gains and incremental costs for each of the three scenarios: (1) optimal utilization rates of both chemopreventive tamoxifen and screening mammography, (2) chemopreventive tamoxifen utilization rates are optimal while the mammography screening rates are 'observed' rates (screening rates observed in WVMP setting), and (3) no tamoxifen is being consumed and mammography screening rates are 'observed' rates. Incremental analysis of scenario 1 over scenario 3 will provide cost per life expectancy gain estimates 
of most optimal scenario (chemopreventive tamoxifen and routine mammography screening as per the ACS screening guidelines) versus maintaining status quo (mammography screening at rates observed in real-world setting). Incremental analysis of scenario 2 over scenario 3 will provide cost per life expectancy gain estimates of most likely scenario (chemopreventive tamoxifen and mammography screening at rates observed in real-world setting) versus maintaining status quo (mammography screening at rates observed in real-world setting).

The incremental cost-effectiveness ratio (ICER) used in the simulation model will be based on the following equation,

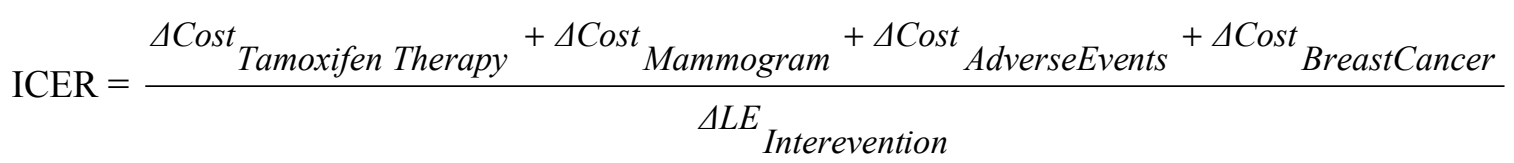

where,

$\Delta \operatorname{Cost}_{\text {Tamoxifen Therapy }}=$ Tamoxifen intervention costs (costs due to use of tamoxifen)

$\Delta$ Cost $_{\text {Mammogram }}=$ Mammography intervention cost (costs due to incremental mammography rates)

$\Delta$ Cost $_{\text {Adverse Events }}=$ Difference in morbidity costs (costs due to increased adverse events) $\Delta$ Cost $_{\text {Breast Cancer Treatment }}=$ Difference in IBC and NIBC treatment related costs $\Delta \mathrm{LE}_{\text {Intervention }}=$ Changes in life expectancy (LE) due to tamoxifen and mammography screening

The numerator in the above ICER equation denotes the change in costs resulting from the intervention. The denominator denotes changes in life expectancy as a result of the intervention.

$\underline{\text { Phase II }}$

Several models exist for breast cancer risk assessment that combine different characteristics of a woman into a qualitative or quantitative risk profile (McTiernan et al., 1997). This study will employ the Gail model (Gail et al., 1989) to assess an individual 
woman's risk of developing breast cancer. The Gail model estimates the probability of a woman with given set of risk factors of developing breast cancer over a specified period of time. It was developed employing data from a subset of the 284,780 women participating in the Breast Cancer Detection Demonstration Project (BCDDP) (Baker, 1982). The risk factors employed in Gail model include family history of breast cancer in first-degree relatives, age at first childbirth, early menarche, and history of previous benign breast biopsies. Atypical hyperplasia, which was later identified as a risk factor, was included in the breast cancer risk estimation in the Gail risk algorithm. The risk factors for breast cancer are discussed in more details in Chapter 2.

The Gail model is considered the best available means of estimating an individual's risk of developing breast cancer. Although few studies have assessed the validity of Gail model in breast cancer risk prediction, the Gail model is often regarded as a 'gold standard' for individual breast cancer risk prediction (Spiegelman et al., 1994). A study by Spiegelman (1994) concluded that Gail model over predicted absolute breast cancer risk. Two other studies (Gail M et al., 1992; Vogel et al., 1993) have validated the Gail model. Both the studies also reported that the Gail model over-predicted breast cancer risk. However, as previously noted, Gail model is the best available means of breast cancer risk prediction, and hence was used for breast cancer risk assessment in this study.

Information on a woman's breast cancer risk factors will be obtained by means of a mail survey to randomly selected WVMP women recipients aged 40 years and above. Information provided by survey respondents on risk factors will be entered into the 'Breast Risk Assessment' program (Gail M et al. model,1989) developed by Cardinal Health System Inc. (Copyright 2000, Cardinal Health Systems Inc.). All women who have a breast cancer relative risk (as calculated by the Gail algorithm) of $\geq 1.7$ will be considered to be at high risk of the disease. The relative risk figure of 1.7 represents the breast cancer risk of a 60 -year old woman with no other breast cancer risk factors other than her age. Subsequent to the individualized risk calculation, the proportion of women at high risk will be determined. The resultant percent value will be extrapolated to the 
WVMP women population 40 years and above to estimate the total number of women in WVMP who will be at high risk of developing breast cancer and thus eligible to receive tamoxifen for chemoprevention of breast cancer.

According to Gail et al., (1992), "there is increasing emphasis on need for counseling to assist women to make informed health decisions." To make such informed health related decisions about preventive services, women should understand and be aware of their own individualized risk of developing breast cancer. Consequently, this study will investigate the relationship between "real risk" as calculated from the Gail model and "perceived risk" of the subject. High correlation will be suggestive of a beneficial trend while low correlation will be a disturbing finding. The latter case demands awareness-enhancing counseling intervention to enable women to make an informed health decision about options that are available to them for 'prevention' of breast cancer. The survey will also obtain information on Medicaid recipient's general knowledge of breast cancer prevention strategies and the levels of compliance with these prevention strategies.

\section{Study Goals and Objectives}

The primary goal of this study is to quantify total costs and consequences of breast cancer preventive strategies in WVMP women enrollees who are healthy but at a high risk of developing breast cancer. Secondary goals of this study include assessment of breast cancer preventive behaviors and perceptions regarding breast cancer prevention management in this population. Specific objectives for Phase I and II of the study are discussed below.

\section{$\underline{\text { Phase I }}$}

The first phase of this study involved development of a Markov model (Beck J et al., 1983) to evaluate the long-term costs and benefits of tamoxifen and mammography screening in prevention of breast cancer. This model was used to calculate the incremental cost-effectiveness ratio (ICER) of introducing tamoxifen in addition to existing mammography screening utilization (optimal screening rates in scenario 1 and 
observed screening rates in scenario two) versus maintaining a status quo (no tamoxifen and observed mammography compliance rates).

\section{Objectives for Phase I}

Objective 1: To develop a Markov process to assess the long term clinical benefits of increased life expectancy from using chemopreventive tamoxifen in conjunction with mammography screening by women who are healthy but are at a high risk of developing breast cancer.

Rationale: The current model follows a hypothetical cohort of women over their lifetime. For health problems that are either chronic in nature or involve events that occur repeatedly over time, a decision tree approach becomes unmanageable. It is difficult to represent events that are repetitive or that occur with uncertain timings using a simple decision tree model. Markov models overcome this hurdle by allowing provisions to incorporate time dependent events.

Objective 2: To develop a cost-effectiveness model based on the Markov process that determines the incremental costs and benefits of using tamoxifen in conjunction with mammography screening versus mammography screening alone in women who are healthy but are at a high risk of developing breast cancer.

Rationale: Rapidly rising costs of medical technologies and services is increasingly reducing the ability to give all patients the care that would benefit them (Brook et al., 1990). The clinical effectiveness of both primary and secondary breast cancer prevention strategies have been well established. However, a decision to implement any such preventive strategies require evidence of not only their clinical effectiveness but also the costs that are incurred due to their implementation (Wright, 1986).

\section{$\underline{\text { Phase II }}$}

The primary goal of phase II was to determine the proportion of women in the WVMP who were at high risk of developing breast cancer. Secondary goals included investigating relationships between women's actual risk and their perceived risk of developing breast cancer, knowledge about breast cancer preventive strategies, current 
usage of preventive strategies and inclination towards using drugs for chemoprevention of breast cancer.

Objectives for phase II

Objective 1: To estimate individual woman's 5-year risk of developing breast cancer in a sample of women aged $\geq 40$ years in the WVMP.

Rationale: Total benefits of a preventive service are dependent, in part, on the baseline risk of population in which those preventive services are being offered (Grann V et al., 2000). Chemopreventive tamoxifen is indicated for reducing risk of breast cancer in only those women who are at high risk of developing breast cancer. Hence, only those Medicaid enrollees who are at high risk for breast cancer will be eligible to receive chemopreventive tamoxifen. Identifying proportion of these high-risk women will help estimate baseline risk of population eligible to receive tamoxifen and assist in quantifying total costs and benefits of introducing chemopreventive tamoxifen in the WVMP.

Objective 2: To assess the sampled women's perceptions of their 5-year and lifetime risk of developing breast cancer.

Rationale: Studies indicate that a woman's perceived risk of developing breast cancer can partly affect her screening practices (Aiken L et al., 1994; Zapka J et al., 1989; Lerman C et al., 1990; McCance K et al., 1990). However, women have unrealistic perceptions about their risk of developing breast cancer (Woloshin et al., 1999). These unrealistic perceptions may generate unnecessary anxiety and may affect appropriate utilization of breast cancer preventive strategies (Lerman C et al., 1995). Consequently, to be able to make informed decisions regarding breast cancer 'prevention,' women need to understand their risk of developing breast cancer (Black W et al., 1995). From a public policy perspective, it then becomes important for policy makers to understand women's perceptions of breast cancer risk so as to be able to communicate more accurate information to these women about their risk. The survey in the present study addresses this issue by estimating the perceived risk of breast cancer in the WVMP.

Objective 3: To assess the sampled women's knowledge of breast cancer prevention strategies

Rationale: Increasing women's knowledge on breast cancer risk and benefits associated with screening may positively influence screening behaviors (Aiken L et al., 1994). 
However, before knowledge enhancing interventions can be designed and implemented, it is important to assess the baseline breast cancer screening knowledge among women in the WVMP. The present study will address this issue of assessing baseline knowledge on breast cancer and its prevention.

Objective 4: To estimate utilization of chemopreventive tamoxifen and assess levels of adherence to mammography screening guidelines among the sampled women.

$\underline{\text { Rationale: }}$ Studies indicate that women in lower income groups tend to under-utilize screening mammography, present with advanced stages of diseases, and subsequently have higher rates of breast cancer attributed mortality (Davis T et al., 1996). The extent of mammography screening under-utilization in the current West Virginia Medicaid women population is unknown and thus needs to be estimated. Mammography screening rates will be assessed through the mail survey.

Objective 5: To assess sampled women's willingness to use tamoxifen for chemoprevention of breast cancer.

Rationale: For high-risk women, tamoxifen has become the latest available option in primary prevention of breast cancer. However, the benefits of tamoxifen in chemoprevention of breast cancer will depend upon its acceptance in the eligible population. Thus, it is important to understand the inclination of this group of high-risk but otherwise healthy women towards taking tamoxifen in primary prevention of breast cancer.

\section{Significance of Research}

According to the United States Preventive Services Task Force, effectiveness of an intervention should be the prime requirement for its acceptance in health care, especially for those interventions that are preventive in nature (U.S. Department of Health and Human Services, 1996). Results of the BCPT trial indicated chemopreventive tamoxifen's ability to significantly reduce the risk of breast cancer in healthy women who are at a high risk of developing breast cancer (Fisher et al., 1998). Ability of mammography screening to detect breast tumors at an earlier stage thereby reducing breast cancer mortality has also been well documented (Costanza, 1992; Shapiro, 1988; Shapiro, 1989; Seidman et al., 1987; Tabar et al., 1992). Hence, based on 
recommendations of the task force, these interventions meet the criteria for their implementation in medical care practice, at least from a clinical perspective. However, in the same context, the task force guidelines also highlighted that effectiveness alone is not a sufficient criterion to initiate services in most practical health care contexts, emphasizing the important role of cost-effectiveness approach in policy decisions.

The significance of cost-effectiveness approach is especially relevant for health insurance systems such as the state Medicaid programs as they provide insurance to populations that are generally in worse health than the general population and rely on Medicaid coverage for their prescription drugs and other preventive services (Bruen B, 2002; CMS, (www.hcfa.gov/medicaid/mservice.html). Consequently, introducing newer interventions in addition to existing ones will strain Medicaid budgets which are already experiencing difficulty due to federal and state budget deficits (Bruen B, 2002). Consequently, there is growing pressure on these agencies to evaluate all new interventions before implementation.

Cost-effectiveness analysis, which combines information on the health benefits, health risks, and costs of health care services, is one such approach that can incorporate and complement evidence on effectiveness for informed policy decision making (U.S. Department of Health and Human Services, 1996). The Phase I of the current study addresses this issue by quantifying and interpreting long term survival benefits and long terms costs of chemopreventive tamoxifen in presence of more traditional preventive strategy, mammography screening. Results from phase I will enumerate additional costs and benefits of chemopreventive tamoxifen and mammography screening, thereby establishing their combined efficiency in delivering health benefits.

However, another issue beyond traditional cost-effectiveness approach is that of affordability (Trueman et al., 2001). Cost-effectiveness of an intervention does not automatically guarantee its affordability for a given health system. In the present context, ability of the WVMP to afford chemopreventive tamoxifen in addition to ongoing mammography screening, will not only depend on tamoxifen's cost-effectiveness profile 
but also on number of women in WVMP who will actually consume the drug. This rate of acceptance will in turn be dictated by two main factors: (1) the proportion of women who are at high risk of breast cancer and thus eligible to receive chemopreventive tamoxifen, and (2) the extent of acceptance of chemopreventive tamoxifen among these high risk women. Currently, no information exists with regards to either of the two stated factors in the WVMP setting. Consequently, phase II of the study was developed in order to assess WVMP women's breast cancer risk profile (and thereby their eligibility to receive chemopreventive tamoxifen) and willingness of these women to consume the drug. Analysis of phase II data will help estimate the proportion of WVMP women population who are eligible to receive chemoprevention as well as their willingness to consume tamoxifen. Combining resultant data with cost effectiveness data from phase I will provide the overall impact (and thus affordability) of introducing chemopreventive tamoxifen in addition to mammography screening to WVMP. 


\section{CHAPTER 2}

\section{REVIEW OF LITERATURE}

Ability to estimate costs and benefits of breast cancer preventive strategies in women who are at high-risk of developing the disease is based on the premise that these subgroup of high-risk women can be appropriately identified. Hence, the first section of this chapter involves detailed discussion of breast cancer risk factors and their use in models for identifying this population of women who are healthy but are at a high risk of developing breast cancer. Clinical effectiveness of breast cancer preventive strategies will be discussed next. Existing literature on economic evaluation of breast cancer preventive strategies in high-risk populations will be critically examined in sections thereafter. Finally, studies investigating relationship between perceived risk of breast cancer, breast cancer knowledge and screening behavior will be reviewed.

\section{Risk factors for Breast Cancer}

Although it is still not possible to exactly predict who will develop breast cancer, there are specific risk factors that place some women at higher risk of developing the disease than others. Some of these risk factors have found wide acceptance among today's scientific community (Vogel, 1991) and include age, family history of breast cancer, prior history of breast cancer, benign breast disease of proliferative patterns or atypical hyperplasia, and endogenous endocrine factors such as menses duration, age of menarche, and late pregnancies (Henderson, 1993). These risk factors are also termed as non-modifiable risk factors as most women have little or no control over them.

Other risk factors for instance, environmental factors, which include dietary habits, alcohol consumption, smoking, exposure to ionizing radiation, use of oral contraceptives, and use of exogenous hormones in estrogen replacement therapy are modifiable. However, a strong association between the modifiable risk factors and breast cancer has yet to be established (Henderson, 1993). 


\section{$\underline{\text { Non-modifiable Risk Factors }}$}

Gender: Being female can be considered to be the single most important risk factor for developing breast cancer. Less than $1 \%$ of all reported breast cancer occur in males (Kopans, 1998).

Age: The probability that a woman will develop breast cancer increases with age, and most breast cancers occur in the postmenopausal years (Henderson, 1993). Thus, besides being female, age might be considered the single most important risk factor for breast cancer. For instance, the risk of a 60 -year old woman developing breast cancer is about 15 times that of a 30-year old woman (Kopans, 1998; American Cancer Society, 20012002; Henderson, 1993).

Family history: Family history of breast cancer, either in the maternal or paternal line, increases the individual's risk of developing breast cancer. Further, a relatively substantial increase in risk is associated with breast cancer in a first degree relative: mother, sister, or daughter and when two or more first-degree relatives have breast cancer, the risk of developing breast cancer is further increased (Henderson, 1993). Prior history of breast cancer: Studies (Kopans, 1998; Henderson, 1993) report that a woman who has already had a breast cancer is at a greater risk of breast cancer recurrence as compared to a woman with no history of breast cancer. Studies report this increased risk in the range of $1 \%$ to $14 \%$ depending upon the period of follow up as the risk increases with time.

Hormonal Status: Woman who have an early menarche or a late menopause are reported to be at a higher risk for developing breast cancer (Kopans, 1998). A study (Kvale G et al., 1988) involving 63,000 Norwegian women, reported that risk increased by $4 \%$ for every year of decrease in the age of menarche between ages 13 and 16. The same study also reported of a $3.6 \%$ increase in risk for each year of delayed menopause. This may be probably related to duration and type of hormonal effects on the breast (MacMohan, 1973).

Age at first full-term pregnancy: Late full term pregnancy is reported to be a risk factor for developing breast cancer. Thus, a woman whose age at first birth was less than 19 years has approximately $50 \%$ the breast cancer risk of a nulliparous woman (MacMohal 
et al., 1970). Moreover, a woman who has her first full term pregnancy by 18 years of age has approximately one-fourth to one-third the risk of developing breast cancer in comparison to a woman whose first full term pregnancy occurs after 30-years of age (MacMohal et al., 1970).

Benign breast disease and atypical proliferative changes: Lack of sufficient data suggests that benign breast disease may not be associated with increased risks of breast cancer. However, benign breast disease accompanied by proliferative changes have been reported to increase risk of developing breast cancer. Moreover, proliferation involving atypical epithelial hyperplasia is associated with significantly increased risk. For instance, women with atypical proliferative changes were 5 times more likely to develop breast cancer than those women with non-proliferative changes (Dupont et al., 1985).

\section{Modifiable Risk Factors}

Use of exogenous hormones: Large numbers of case-control studies concerning estrogen replacement have been conducted over the years. However, the results of these studies have been contradictory. Some studies have shown a visible increased risk after use of estrogen replacement, while others have shown no such increase. In fact, some studies have actually found a decrease in breast cancer risk (Henderson, 1993). Effect of use of oral contraceptive use on risk of developing breast cancer has also been studied using meta-analysis. When considered together, these studies indicate no significant increase in risk of breast cancer with use of oral contraceptives in the case-control studies (Romieu et al., 1990). However, according to Henderson (1993), prolonged use of an oral contraceptive might be associated with increased risk of breast cancer.

Environmental factors: There is strong evidence that one or more environmental factors are associated with risk of breast cancer. Dietary habits, which is an environmental factor, however, has received the greatest attention (Henderson, 1993). Alcohol intake has been very strongly associated with breast cancer risk with studies showing strong correlation of breast cancer risk and alcohol intake (Longnecker, 1988). 


\section{Models for Assessing Individual Risk for Breast Cancer}

There has been an increased demand for methods assessing breast cancer risk as women, medical professionals, and decision makers are becoming more aware of factors that might affect risk for breast cancer (McTiernan et al., 1997). Risk assessment for breast cancer involves identifying certain factors in an individual woman that are pertinent to her risk, and combining those factors into a quantitative or qualitative profile (McTiernan et al., 1997). Significant numbers of models have been developed to assess these risks. Some of the important models will be discussed in this section. The models predicting breast cancer risks can be categorized based on the methodology that was employed in risk determination. Thus, some models incorporate empirical estimates, others employ statistical modeling, while still others employ epidemiological literature review in breast cancer risk assessments. A review of these models revealed that all the stated models include at least the age and the family history as risk factors for predicting individual risk for breast cancer.

\section{$\underline{\text { Genetic Susceptibility Models }}$}

These models derive the breast risk algorithms based on evidence of rare autosomal dominant allele that results in increased susceptibility to breast cancer. Models such as Ottman et al., (1983), Anderson et al., (1985), and Claus et al., (1994) fall in this category.

\section{Ottman et al., (1983) model}

This model uses life table analysis (cumulative risk) of a population-based series of breast cancer patients and their relatives to estimate the probabilities of developing breast cancer. The cases included white women diagnosed between 1971 and 1975 with unilateral or bilateral breast cancer before age 65 .

Anderson et al., (1985) model

This model examined 556 breast cancer pedigrees, and empirically calculated risks for breast cancer among relatives of cases. The population for the model involved women between 20 to 70 years. The factors for risk assessment included breast cancer in mothers, sisters, second-degree relatives, age of onset in relatives, and laterality. This model was intended to be used for genetic counseling. 
Claus et al., (1994) model

This model employed statistical modeling in breast cancer risk assessment. The model included 4,730 breast cancer cases and 4,688 controls aged 30 to 54 years derived from Cancer and Steroid Hormone Study (McTiernan et al., 1997). This model incorporated the autosomal dominant major gene model to predict breast cancer risk for relatives of breast cancer patients (Claus et al., 1994). Factors for predicting breast cancer risk in this model included age, breast cancer in first and second-degree relatives, and age of onset in relatives. Similar to above two models, this model is intended for genetic counseling.

\section{$\underline{\text { Risk-based Categorization Models }}$}

Taplin et al., (1990) model

This model used a risk-based selective approach to screening and was developed in a health maintenance organization with 400,000 members. It places women in one of the four risk categories depending upon the individual woman's constellation of risk factors (Taplin et al., 1990). The risk factors for the model were obtained from literature review and included age, family history of breast cancer, benign breast disease, age at menarche, age at menopause, age at first live birth, and nulliparity. This model has been proposed to be used for screening decisions. The model included women of all ages.

Gail model and National Surgical Adjuvant Breast and Bowel Project's (NSABP) adaptation of the Gail model

Details of Gail model are described in Chapter 3. Validation of the original Gail model revealed that the model over-estimated absolute risk by about $40 \%$ in women below age 55 in the general population where regular screening is not performed (Gail $\mathrm{M}$ et al., 1992). Consequently, the NSABP's Breast Cancer Prevention Trial used an adapted version of Gail model (1989) in which the 1983-1987 average annual breast cancer rates were substituted by the baseline incidence from the Breast Cancer Demonstration Project (BCDP), and 1988 U.S. mortality rates for all causes other than breast cancer was substituted the 1979 mortality rates originally used in Gail model.

As mentioned in Chapter 1, this study will make use of NSABP adapted version of Gail model in order to minimize the breast cancer risk over-estimating nature of 
original Gail model. Moreover, Gail model is considered to be a "gold standard" in breast cancer risk assessment (Spiegelman et al., 1994). Finally, the proposed application of the Gail model is in concordance with the main objective of phase I of this study, which is to determine the proportion of high risk population.

\section{Prevention Management of Breast Cancer}

Evidence for Clinical Efficacy of Primary Prevention Strategy

Tamoxifen is one of the first therapies to be investigated in the primary prevention of breast cancer. Newer agents such as raloxifene are being developed and tested for their potential in chemoprevention of breast cancer. However, these agents are still under investigation and currently no concrete data exists to validate their potential in primary prevention of breast cancer. Tamoxifen has been widely used for more than 20 years as an adjuvant therapy in treatment of breast cancer. Studies using animal models have indicated that tamoxifen affects both the initiation and promotion of tumors (Terenius L, 1971; Jordan V, et al., 1980).

Furthermore, evidence on effectiveness of tamoxifen in reducing mortality and recurrence in women with early stage breast cancer has been well established through numerous trials, a review of which has been presented in the article by the Early Breast Cancer Trialists' Collaborative Group (EBCTCG, 1998). Other studies evaluating tamoxifen as an adjuvant therapy for breast cancer found that the incidence of contralateral breast cancer was reduced among patients who received tamoxifen (Noe et al., 1999). These trial results along with studies in animal models led to development and initiation of the NSABP's Breast Cancer Prevention Trial (BCPT) protocol P-1 (Fisher et al., 1998).

For this double-blind placebo-controlled multi-center randomized clinical trial, women with high risk of breast cancer $(n=13,175)$ were enrolled at 131 sites in United States and Canada. The eligibility criteria included women: (1) those older than 60 years; (2) those with history of lobular carcinoma in situ; and (3) those with a $>1.6 \%$ risk of the development of breast cancer in 5 years based on a breast cancer risk algorithm 
developing by Gail and colleagues (Gail et al., 1989), predicting a woman's risk of development of breast cancer. The subjects received tamoxifen $20 \mathrm{mg}$ per day or placebo for an average of 47 months. Multiple outcomes were measured in this trial which allowed quantification of both benefits as well as risks associated with tamoxifen.

Tamoxifen was found to reduce the relative risk of invasive breast cancer by about $49 \%$ in woman of all age groups ( 35 to 49,50 to 59 , and 60 and above). It also reduced the relative risk of noninvasive breast cancer by about $50 \%$. However, women above 50 years of age who were treated with tamoxifen had a greater relative risk for developing endometrial cancer $(\mathrm{RR}=4.01)$ and pulmonary emboli (Relative Risk $(\mathrm{RR})=$ 3.19). Tamoxifen treated women also developed more cataracts $(R R=1.14)$ and underwent more cataract surgeries $(\mathrm{RR}=1.57)$ than their counterparts who received placebo. In tamoxifen treated women older than 50 years of age, there were fewer fractures of the hip, wrist and spine $(R R=0.79)$ and more stroke events $(R R=1.75)$ and deep vein thrombosis $(\mathrm{RR}=1.71)$. However, frequency of these events was not significantly different than in women who were in the placebo group.

Tamoxifen's role in primary prevention of breast cancer was also studied in two European trials (Powles et al., 1998, Veronesi et al., 1998). The British trial by Powles and colleagues (1998) involved 2,494 healthy women aged between 30 to 70 years with family history of breast cancer. The experimental group received 20-mg tamoxifen per day with the primary outcome being occurrence of breast cancer. The median follow-up for this trial was 70 months. The overall frequency of breast cancer was found to be similar in both groups. The trial failed to show any effect of tamoxifen on breast cancer incidence in healthy women. The Italian trial by Veronesi and colleagues included 5,408 hysterectomised women between ages of 35 to 70 years. The experimental group received the standard 20-mg per day dose of tamoxifen. The median follow-up for this trial was 46 months. Once again, no significant difference between tamoxifen and placebo groups was observed in terms of breast cancer occurrence. 
Differences in power to detect reduction in breast cancer incidence may have a partial role in explaining conflicting results between the NSABP's BCPT trial and the two European trials (Pritchard K, 1998). The European trials were much smaller and accumulated only a little more than two-thirds of the women-years of follow up in BCPT. Further, baseline breast cancer incidence among the placebo group in the Italian trial was significantly lower than in BCPT. It is suggested that higher population base coupled with greater baseline breast cancer incidence in the BCPT is more conducive in terms of demonstrating an effect.

Furthermore, differences in drug compliance, population age, and genetic differences in the population selected in the trials may have contributed to the outcomes. For instance, in the Italian trial as high as one-fourth of the study population dropped out within one year of trial. For other women who continued to remain in the trial for more than a year, a preventive effect was close to achieving a significance level $(p=0.16)$. Women included in the British trial tended to be younger than the ones enrolled into BCPT. For instance, about $60 \%$ of women were aged under 50 years, as compared to 40 $\%$ in BCPT trial. Since all the preventive effect of tamoxifen in the BCPT was limited to estrogen-receptor positive tumors, which tend to occur more in older women, age differences between trials can be a factor influencing significance. Strong family history of breast cancer, a main eligibility criterion in the British trial, was different than eligibility rules set in BCPT trial, which were mostly non-genetic in nature. It is suspected that prevention effect of tamoxifen may be different among these two populations.

The BCPT trial had higher power and high internal consistency, and had an acceptable drug compliance rate. Moreover, the percent breast cancer risk reduction observed in the BCPT trial was very similar to preventive effect observed in the Early Breast Cancer Trialists Collaborative Group (EBCTCG) trials (EBCTCG, 1998). Finally, the USFDA approval for tamoxifen in breast cancer risk reduction was based on results of NSABP's BCPT trial, which involved the North American population, a population 
similar to the one that is being considered in the present study. Hence, results from the NSABP's BCPT trial will be employed in the current study.

\section{Evidence for Clinical Efficacy of Secondary Prevention Strategy}

The following discussion involving trials on efficacy of mammography screening is restricted to randomized controlled trials (RCTs) which involved random division of women population into two groups, one that was offered screening while other that did not and served as unscreened control. A total of nine RCTs have been conducted since the early 1960s in United States, Canada, and Europe.

Among the first screening trials was the Health Insurance Plan of the New York trial, which investigated whether screening asymptomatic women for breast cancer using mammography in conjunction with CBE could lower the death rates (Kopans, 1998). The trial involved 62,000 women between ages of 40 and 64 years. The trial demonstrated a mortality reduction of $23 \%$ in screened women, which appeared about 3 to 5 years after the first screen (Shapiro, 1988).

The European trials evaluated efficacy of mammography in absence of CBE. The first two Swedish trials also known as the Two County Trial (Koppaberg and Ostergotland trials), were separate trials, although their results were presented together (Nystrom et al., 1993). The combined study population of two trials was 134,867 and the women involved were between ages of 40 and 74 years. The trial results demonstrated a $30 \%$ mortality reduction for screened population (Tabar et al., 1985).

Another RCT in Malmö, Sweden involved 42,000 women aged 45 to 69 years old (Anderson et al., 1988). Results from early follow-up indicated a $20 \%$ mortality reductions in women 55 years and older, although these reductions did not reach statistical significance. A ten-year follow up indicated a high mortality reduction of $49 \%$ among women between ages of 45 to 49 years. A separate Stockholm trial, which included 38,525 women aged 40 to 65 years old reported a mortality reduction of $21 \%$, which did not achieve statistical significance owing to relatively small number of women 
enrolled in the trial (Frisell et al., 1986). The Gothenburg trial included 52,000 women aged 40 to 59 years old. The results from the trial indicate a $14 \%$, non-significant mammography-screening attributed mortality reduction (Kopans, 1998).

A RCT in Edinburgh involved 45,130 women between ages of 45 to 64 years (Roberts et al., 1990). The results of this trial indicate almost $20 \%$ relative reduction in mortality after 7 to 9 years of follow-up. Once again, due to small size of the trial the results did not achieve statistical significance.

The National Breast Screening Study of Canada trial composed of two sub-trials. The first determined efficacy of screening in women aged 40 to 49 years (Miller et al., 1992(a)) while the second trial determined efficacy in women aged between 50 and 59 years (Miller et al., 1992(b)). Results from the former trial failed to demonstrate significant mortality reduction between study and control groups. The second trial evaluated individual contribution of mammography screening in mortality reduction by comparing study group, which received both annual mammography and CBE, and control group that only received annual CBE. As the second trial did not have an unscreened control group, it could not be determined if there was any benefit for the women participating in the trial from either approach to screening (Kopans, 1998).

In summary, these trials do indicate efficacy of mammography screening and its potential in reducing death rate through routine screening (Fletcher, 1993). A metaanalytic study, which combined results from all of the trials, indicated survival benefits from screening (Elwood et al., 1993). Based on updates of the seven RCTs (the two NBSS trials were excluded), Wald and colleagues calculated a overall $22 \%$ mortality reduction though screening women 40 to 74 years old (Wald et al., 1994).

Controversy, however, exists regarding screening effectiveness in women younger than 50 years of age. However, evidence from a meta-analytic study involving combined results from seven randomized trials that included women aged 40 to 49 years suggests a statistically significant mortality reduction of $24 \%$ among women in this age 
group who were offered screening for breast cancer (Smart et al., 1995). Adding to this evidence are two separate Swedish trials, which have shown statistically significant reductions in breast cancer mortality for women in their forties (Hendrick et al., 1997). In addition, these studies show that more frequent screening is significantly more effective than biennial screening for women aged 40 to 49 years (Report of the organizing committee and collaborators, 1996; Bjurstam $\mathrm{N}$ et al., 1997).

\section{Economics of Prevention Strategies}

Effectiveness alone is not sufficient in most cases to warrant implementation of clinical services. Other factors such as short term trade-offs and long term consequences resulting from the clinical services should also be factored-in during the decision making process (Siegel J et al., U.S. Preventive Services Task Force). Among widely used approaches to quantify these trade-offs in terms of costs and consequences are through use of decision-analytic modeling like decision trees and Markov models. Following is a brief discussion providing rationale of choosing Markov model over decision tree approach in the current study.

Although convenient, application of decision tree is mostly limited to situations involving events that occur once over a relatively short period of time. For health problems that are either chronic in nature or involve events that occur repeatedly over time, decision tree approach becomes unmanageable. It is difficult to represent events that are repetitive or that occur with uncertain timings using a simple decision tree model. Markov modeling overcomes this hurdle by allowing provisions to incorporate time dependent events.

The model assumes that an individual is always in one of the finite number of states of health referred to as the Markov states. All events are then based as transitions of individuals from one state to another. Individuals make these transitions during each cycle, which are equal increments of time horizon of the analysis. The cycles are model specific. For instance, a unit cycle may constitute one year for one model and one month for other depending upon the clinical problem under consideration. 
The transitions between Markov states are governed by pre-specified probabilities that are input in the model. These probabilities are known as transitional probabilities. Each state is assigned a unique utility value. The utility contribution of this state to the overall utility then depends on time that an individual spent in that particular state. For instance, if all that needs to be calculated is a patient's life expectancy, then utility of all non-dead states can be assigned 'one'. There is a state in Markov model, which is called the absorbing state. Once an individual transits into this state, he or she cannot leave this state. For instance, DEAD state is an absorbing state in many Markov models. Once an individual is in the DEAD state, he or she remains in this state forever. The absorbing state depends upon the effectiveness endpoint of the study and need not be 'Death' for all models.

In general, two types of Markov models can be employed in medical decision making. In the first type, the transition probabilities are constant or non-time dependent while in the second type the transition probabilities vary over time. The former types of models are 'Markov chains' while the latter types are 'Markov processes.' Markov chains have somewhat limited applicability in that they can be applied only to those clinical problems in which likelihood of moving between the health states can be assumed to be constant. Constant transition probabilities are only possible for clinical problems with short time horizons. In more chronic diseases, probabilities of moving between states vary over time and thus are time-dependent (Beck J et al., 1983). For instance, there is an exponential increase in annual mortality of healthy population with increasing age (Gompertz B, 1825; Pauker S et al., 1981). This translates into an increasing time-dependent probability of transiting into the 'Death' state.

Rising health care expenditures coupled with limited resources have spurred interest in evaluating new and existing health services both in terms of their benefits and costs. The next two sections include discussion about studies that have investigated economic efficiency of breast cancer prevention techniques. 


\section{Economic Evaluation of Primary Prevention Strategy}

To date, only three studies have investigated the economic potential of tamoxifen in chemoprevention of breast cancer. The first study, by Smith et al., (2000) assumed the perspective of a health service payer and compared tamoxifen to no therapy. Results of the NSABP's BCPT (Fisher et al., 1998) were used to determine the cost-effectiveness of tamoxifen. Cost data was obtained from the Agency for the Health Care Policy and Research. The economic analysis evaluated the direct costs for the period of 5 years of trial. The study reported that the cost to reduce the risk of invasive breast cancer for one woman would be about $\$ 292,523$. Assuming breast cancer deaths could be entirely prevented by 5 years of tamoxifen, then women in their early forties would gain about 34.5 years of life at a $3 \%$ discount rate. This translates into a cost-effectiveness ratio of $\$ 8,479$ per additional year of life gained.

The above study, however, made assumptions, which markedly differed, from real world experiences. For instance, the study assumed that benefits of tamoxifen with regards to reduction in breast cancer risk lasted a lifetime. This is in contrast to data derived from a recent evaluation of use of adjuvant tamoxifen for treatment of breast cancer in more than 37,000 women in 55 trials, which suggests that the protective effect of tamoxifen may last at most for only about 5 more years in addition to the 5 year time when the drug is being taken (EBCTCG, 1998).

A second study by Noe L et al., (1999) used a decision analytic model to estimate the age-based incremental cost-effectiveness of using tamoxifen compared to no intervention in primary prevention of breast cancer. A multi-stage Markov model was developed to evaluate the incremental cost-effectiveness. The model was populated based on data derived from the BCPT trial. Different health states involved in the Markov model construction included invasive and non-invasive breast cancer, endometrial cancer, pulmonary embolism, and cataract surgery. The economic evaluation was performed from the payer's perspective and hence out-of-pocket costs, costs due to lost productivity and time lost from work and non-medical costs were not 
calculated in the study. Cost parameters were obtained from published literature and were adjusted to reflect 1997 dollars. The primary effectiveness measure of the analysis was additional life years saved and the cost-effectiveness ratio depicts additional lifetime costs required to gain one extra year of life with tamoxifen in comparison to no therapy. The cost and benefits were discounted at the rate of $3 \%$ in the base case analysis. Assuming a 5-year breast cancer protective effect of tamoxifen, it was reported that for women in general regardless of age, the incremental cost per life year gained was $\$ 63,896$. However, this cost-effectiveness ratio varied across different age groups. For women between ages of 35 and 49 years, the incremental cost per life year gained was $\$ 41,372$. The cost-effectiveness ratio increased for higher age groups and was $\$ 68,349$ per life year gained for women between ages of 50 and 59 years and $\$ 74,981$ per life year gained for women between 60 and 69 years. When the assumption of tamoxifen's protective effect was increased to a total of 10 years, lower cost-effectiveness ratios of $\$ 20,806, \$ 36,431$, and $\$ 41,621$ per life year gained were reported for women belonging to $35-49,50-59$, and 60-69 year age-groups, respectively.

The third study by Grann and colleagues was similar to the one just discussed. A computer-based decision analysis, involving Markov model with Monte Carlo simulations, was employed to construct the study model (Grann et al., 2000). The costeffectiveness results were adjusted for quality of life by including utility measures which were obtained by administering a time-trade off questionnaire to a group of community based women. Although, the Markov model constructed had similarities to Noe et al., (1999), it differed in terms of health states. Different health states included in the model were good health, good health after stopping tamoxifen, invasive and non-invasive breast cancer, hip fracture, thrombophlebitis, endometrial cancer, cataracts, and death. The study assumed a total of 5-year breast cancer protective effect of tamoxifen. The base case discount rate of $3 \%$ was used in the analysis. The study reported that for women who start using tamoxifen at age 35 , the cost-effectiveness ratio is $\$ 46,619$ per life year saved. For women who start taking tamoxifen at age of 50 and 60 have costeffectiveness ratio of $\$ 82,784$ and $\$ 122,401$ per life year saved, respectively. The quality 
adjusted cost-effectiveness ratios were $\$ 76,318, \$ 130,660$, and $\$ 142,227$ per quality adjusted life years for women starting at age 35, 50 and 60 years, respectively.

The above two studies evaluated economic potential of chemopreventive tamoxifen in isolation in the sense that they failed to include either benefits or costs of mammography screening technique. As will be evident from the following discussion on economic evaluation of secondary preventive strategy, mammography screening on its own entails substantial cost but delivers benefits independent of chemopreventive tamoxifen. Hence, any attempt to quantify economic efficiency of breast cancer preventive strategies should not only include the primary strategies like chemopreventive tamoxifen but also secondary strategies such as mammography screening. Moreover, these trials also failed to factor in drug compliance, which is an important issue for chronic therapies like chemopreventive tamoxifen that lasts for 5 years.

\section{Economic Evaluation of Secondary Prevention Strategy}

There have been numerous studies involving economic evaluation of mammography screening. Economic evaluation of mammography screening have compared different aspects of screening such as differences in screening interval, age range of women undergoing screening, compliance with screening, and proportion of women who show abnormalities and consequently have to undergo follow-up tests. The following discussion will review some of these studies so as to provide an overview on the economics of screening effectiveness for different screening intervals and across different age-groups of women undergoing mammography screening.

A literature review of cost-effectiveness studies reveals significant variations in the cost-effectiveness ratio for mammography screening. Part of these differences have been due to difference in mortality reduction attributed to mammography screening, and failure on part of some economic studies to include time lag between actual initiation of mammography screening and time when benefits in terms of mortality reduction start accruing (Feig S, 1995). Despite these differences in results, the cost-effectiveness of mammography screening ranges from $\$ 3,400$ to $\$ 28,700$ in general. 
A study by Salzmann et al., (1997) compared cost-effectiveness of screening women in different age groups. Different mammography screening interventions compared were: (a) biennial screening from 50 to 69 years of age compared to no screening, and (b) screening women between ages of 40 to 49 years every 18 months followed by biennial screening from ages 50 to 69 years was compared to biennial screening from ages 50 to 69 years. A Markov model was used to compare life expectancy of women undergoing different breast cancer screening strategies. The Markov health states included healthy, developed breast cancer and remained alive, dying of breast cancer, and dying of another cause. The strategy, which involved screening women between 55 to 69 years of age, resulted in increased life expectancy by 12 days. This improved life expectancy came at the cost of \$704 per woman translating into a cost-effectiveness ratio of $\$ 21,400$ per year of life year saved. The study also reported a high incremental cost-effectiveness of $\$ 105,000$ for extending mammography screening to women between ages of 40 to 49 years.

A similar study by Rosenquist and Lindfors (1998) assessed cost-effectiveness for four different strategies: (a) screening women annually from ages 40 to 79 years, (b) screening women annually from 40 to 64 years and biennially from ages 50 to 79 years, (c) screening women annually from ages 40 to 49 years and biennially form ages 50 to 79 years, and (d) screening women annually from ages 40 to 49 years followed by annually from ages 50 to 79 years in normal risk women. Markov model was employed in order to estimate the marginal cost-effectiveness of individual strategies. The outcome of interest was marginal cost per year-life saved (MCYLS) and ranged from $\$ 18,800$ to $\$ 16,100$. The authors concluded that MCLYS for all evaluated strategies were well within accepted ranges. 


\section{Perceptions of Breast Cancer Risk, Knowledge, and Preventive Service Utilization}

To be able to make informed decisions regarding utilization of preventive strategies, women need accurate information about their individualized risk of developing breast cancer and effectiveness of different breast cancer preventive strategies (Black W et al., 1995). Similarly, from a clinical practice standpoint, understanding a woman's risk perception is essential for risk management and decision making.

Many studies have investigated relationships between breast cancer risk perception, knowledge, and screening behavior. Literature suggests that women's perception of breast cancer risk remains imprecise (Hopwood P, 2000). For instance, it has been reported that prior to undergoing genetic risk counseling, only a small proportion of women had an accurate view of their risk of developing breast cancer. Moreover, a majority of women either over- or under-estimated their risk of developing the disease (Evans D et al., 1993; Cull A et al., 1999). The extent of over-estimation of risk was found to be greater in studies that were conducted in US (Lerman C et al., 1994; Lerman C et al., 1995). Furthermore, it was reported that women who were actually at a high risk of developing breast cancer, underestimated their risk as compared to woman of average risk (Hopwood P, 2000).

A study involving 500 economically disadvantaged women reported that a large majority of respondents did not perceive themselves to be susceptible to breast cancer. The same study also reported that those who were most knowledgeable about breast cancer were more likely to perceive themselves as being more susceptible to breast cancer than their less knowledgeable counterparts (Price J, 1994).

A meta-analytic review investigated the relationship between a woman's risk and likelihood of obtaining mammography screening (McCaul K et al.,,1996). The study reported positive correlation between perceived risk and undergoing mammography screening. Another study involving middle class women aged 37 to 77 years reported positive relationship between mammography screening and perceived susceptibility. 
Although a lot of studies have investigated the relationship between risk, knowledge and screening behavior, very few studies have investigated this relationship in populations similar to the one being currently studied, WV Medicaid. Moreover, as chemoprevention in breast cancer using drugs like tamoxifen is a fairly recent phenomenon, only a few studies have investigated the relationship between risk, knowledge and inclination towards using such drugs. A study by Cyrus-David et al., (2001) involved a focus group of 26 women with an elevated risk for breast cancer. It assessed the knowledge and attitudes of these women towards the use of SERMs, such as tamoxifen and raloxifene. Knowledge of breast cancer risk factors and the perception of personal risk for breast cancer were reported to influence the acceptance of breast cancer chemoprevention treatment with selective estrogen receptor modulator (SERM). A thorough review of literature at the time of this study revealed no study assessing these relationships in socio-economically under-privileged populations like the Medicaid population.

Mammography screening has been employed in breast cancer screening for over twenty years and its effectiveness in detecting breast tumors early has been well documented. A large number of studies have evaluated mammography screening from an economic perspective and most of these studies have found the screening to be costeffective. Tamoxifen's effectiveness in reducing risk of developing breast cancer has also been shown. However, few studies have evaluated the economic effectiveness of chemopreventive tamoxifen. Lastly, no study has evaluated the combined economic potential of chemopreventive tamoxifen and mammography screening in breast cancer prevention. The next chapter provides objectives for both phase I and II of this study along with detailed methodology that will be employed in order to achieve the study objectives 


\section{CHAPTER 3}

\section{METHOdOLOGY}

The study will be conducted in two phases. Phase I involves development of a life expectancy model with an economic component while phase II involves a selfadministered mail survey to assess West Virginia Medicaid Program (WVMP) women enrollees' actual and perceived breast cancer risk, breast cancer knowledge, current utilization of breast cancer preventive services, and willingness towards chemoprevention.

The objectives of the study were: (1) To develop a Markov process in order to assess the long term clinical benefits of chemopreventive tamoxifen in conjunction with mammography screening in women at high risk of breast cancer, (2) To develop a costeffectiveness model in order to determine the incremental costs and benefits of introducing chemopreventive tamoxifen in conjunction with mammography screening, (3) To assess WVMP women's actual and perceived risk of developing breast cancer, (4) To assess WV Medicaid women's knowledge of breast cancer prevention strategies and their compliance with these strategies, and (5) To assess sample women's willingness to use tamoxifen for primary prevention of breast cancer.

\section{Phase I}

\section{Design of the Model}

A Markov process with time- and state-dependent transition probabilities was developed. DATA 3.5 TreeAge ${ }^{\mathrm{TM}}$ software (TreeAge Software, Inc., 1999) was used to develop the model. Incremental costs and consequences of using chemopreventive tamoxifen and mammography in women at high risk for developing breast cancer was determined using this model. The model incorporated all relevant costs and benefits that were significantly affected by chemopreventive tamoxifen and mammography screening. 
The intervention was modeled based on its impact on disease risks and disease mortality. More specifically, the breast cancer prevention effectiveness of tamoxifen was modeled as decreased risk of developing invasive breast cancer (IBC) and non-invasive breast cancer (NIBC). Similarly, the secondary preventive effectiveness of mammography screening was modeled as reduction in breast cancer mortality over a period of time. Detailed discussion on modeling these interventions is presented later in this chapter.

Costs included in the model were tamoxifen therapy cost, cost of mammogram including follow up costs resulting from abnormal mammogram findings, and cost of treating tamoxifen-related adverse events such as endometrial cancer (EC), pulmonary embolism (PE), and cataract surgeries (Cat-S).

A relatively short-term effectiveness outcome such as "number of breast cancers averted" could have been incorporated in the current model. However, as the model incorporates consequences for multiple diseases, short-term outcomes may not provide meaningful information (Zethraeus $\mathrm{N}$ et al., 2000). A more composite outcome measure of effectiveness is needed. This measure must account for both positive and negative aspects of an intervention. Life-expectancy gain estimation is one such composite outcome measure and was therefore incorporated in the current model as the effectiveness outcome.

\section{Operationalizing the Model}

Tamoxifen therapy in primary prevention of breast cancer lasts for 5 years (Fisher B et al., 1998). Consequently, the time frame (treatment duration) of the current study was five years so as to coincide with the duration of chemoprevention tamoxifen therapy. According to the American Cancer Society (ACS), all women after the age of 40 should undergo mammography screening every year (American Cancer Society). These criteria regarding chemopreventive tamoxifen therapy and mammography screening were incorporated in the two tamoxifen intervention arms of the model. In the first intervention arm, total compliance with mammography screening was assumed while in 
the second intervention arm mammography compliance rates were adjusted to reflect those that were observed in real world setting. Women in the non-intervention arm of the model did not get chemoprevention tamoxifen and underwent mammography screening at 'observed' rates.

The chemopreventive tamoxifen and mammography screening intervention was modeled based on its impact on disease risks and associated mortality. Tamoxifen and mammography screening impact the natural history of disease differently. Tamoxifen reduces incidence of breast cancers, thereby reducing the number of new breast cancers and thus indirectly reducing breast cancer mortality in the high-risk population. Mammography screening, on the other hand, detects some proportion of breast cancers at an earlier stage, thereby improving both prognosis and survival of breast cancer patients (Kopans, 1998). Hence, these two interventions of disease risk and mortality were modeled in distinct ways.

\section{Operationalizing the Model for Tamoxifen Intervention}

Tamoxifen's role in primary prevention of breast cancer was studied in the Breast Cancer Prevention Trial (BCPT) (Fisher B et al., 1998). The subjects received tamoxifen or placebo $20 \mathrm{mg}$ per day for an average of 47 months in this placebo-controlled randomized trial. Multiple outcomes were measured in this trial which allowed quantification of both benefits as well as risks associated with tamoxifen.

Trial results showed that tamoxifen reduces risk of IBC and NIBC and increases risk of other diseases such as EC, PE, and cataract (Fisher B et al., 1998). Tamoxifen reduced the risk of IBC by $49 \%$ and NIBC by $50 \%$. This risk reduction was observed in all age groups. Tamoxifen significantly increased risk of other events: EC by 2.53 times, PE by 3.0 times, and cataracts by 1.14 times. Frequencies of other events such as coronary events, or bone fractures were not significantly different in the tamoxifen and placebo groups (Sweeney F et al., 1999). 
Tamoxifen's impact on risk of different diseases was then operationalized in the model using the trial results. Event rates obtained from BCPT could not be directly used in the model as rates by definition represent "pressure to transit at a given point in time" (Miller D et al., 1994). Hence, the 'rates' were converted to 'risks' (probabilities) which represent actual transitions by individuals in a cohort over a period of time (Miller D et al., 1994). There are multiple ways to derive risks from rates: the simple cumulative method, the actuarial method, and the density method. The density method was used to convert rates into risks. This method uses estimated group-specific incidence densities (rates) to calculate risk for a specific group or time-interval (Miller D et al., 1994).

Thus, when instantaneous transition rate remains constant during the entire time period, the $\Delta$-year risk, $\mathrm{P}\left(\mathrm{t}_{0}, \mathrm{t}\right)$ is determined by:

$$
\mathrm{P}\left(\mathrm{t}_{\mathrm{o}}, \mathrm{t}\right)=1-\left(\mathrm{N}_{\mathrm{t}} / \mathrm{N}_{0}\right)=1-\exp [-\operatorname{ID}(\Delta)]
$$

Where,

ID = estimated average rate

$\Delta=$ elapsed time $\left(\mathrm{t}-\mathrm{t}_{0}\right)$

This formula was employed in order to determine transition risks (probabilities).

\section{Operationalizing the Model for Mammography Screening Intervention}

As per the ACS breast cancer screening guidelines, this study assumes that all women entering the cohort will be eligible candidates for routine mammography screening (as women enter the cohort at age of forty). Mammography screening trials failed to show mortality reductions for 7 to 9 years of screening in women between ages of 40 to 49 years (Kerlikowske K et al., 1995; Elwood J et al., 1993; Glasziou P et al., 1995; Nystrom L et al., 1993). However, a study reported a statistically significant $16 \%$ mortality reduction (Kerlikowske K, 1997) after 10 to 14 years of initiation of screening (Kerlikowske K et al., 1995; Nystrom et al., 1993; Tabar L et al., 1992). This aspect of delayed benefit of mortality reduction associated with beginning mammography screening at age 40 was incorporated into the model. 
Another study, which estimated efficacy of mammography screening, indicated a $27 \%$ mortality reduction in women who began screening at 50 years of age (Kerlikowske $\mathrm{K}$ et al., 1995). In this case there was a delayed benefit of 5 years after initiation of screening (Kerlikowske K et al., 1995; Nystrom et al., 1993; Tabar L et al., 1992).

Thus, in the current model, women who began screening at 40 years of age will experience a $16 \%$ mortality reduction benefit of screening at age 50 years (a 10-year delayed benefit). At age 55, this increases to $27 \%$ (5 years delayed benefit). This modeling approach was based on a previous study evaluating cost-effectiveness of extending mammography screening to include women 40 to 49 years of age (Salzmann $\mathrm{P}$ et al., 1997).

\section{Operationalizing the Model for Mortality}

The BCPT trial was not designed to determine mortality difference between treatment groups. Furthermore, the follow up period of the trial was not long enough to determine impact of tamoxifen-associated risk alterations of different events such as IBC, NIBC, EC, PE, DVT, and cataract on mortality. Hence, mortality data obtained from various published sources (details on these sources are discussed later in the section titled 'Data for the model') were used in order to determine annual risk of mortality from the above mentioned events. Mortality rates were adjusted for each diseased and nondiseased state. These mortality rates, then, were further adjusted to reflect age-sex-race (ASR) dependent mortality.

\section{Model Structure and Simulation}

The model structure is illustrated in Figure 3.1. 
Figure 3.1: The Transition State Diagram

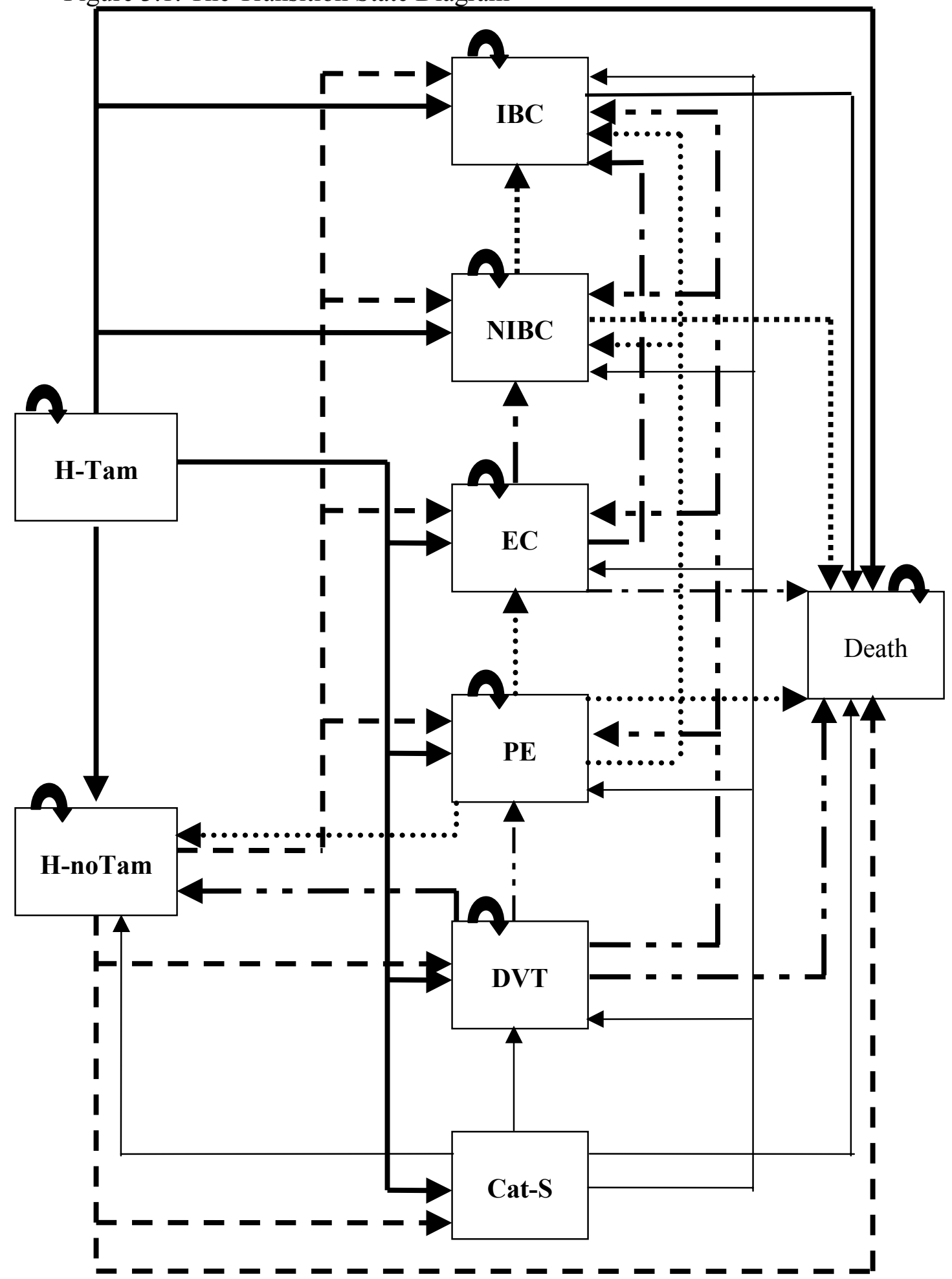

H-Tam: Healthy with Tamoxifen; H-noTam: Healthy with no Tamoxifen; IBC: Invasive breast cancer; NIBC: Non-invasive breast cancer; EC: Endometrial cancer; PE: Pulmonary embolism; DVT: Deep vein thrombosis; Cat-S: Cataract surgery 
The model illustrates the possible transition of individuals between the distinct health states. The model comprises of 9 distinct health states: 1 . Healthy with tamoxifen (H-Tam), 2. Healthy without tamoxifen (H-noTam), 3. Invasive breast cancer (IBC), 4. Non-invasive breast cancer (NIBC), 5. Endometrial cancer (EC), 6. Pulmonary embolism (PE), 7. Deep Vein Thrombosis (DVT), 8. Cataract surgery (Cat-S), and 10. Death.

At any given point in time, an individual woman could reside in one of the 9 states. Change of state, or transitions, that occurred over a fixed time interval are illustrated as arrows in the Figure 3.1. All women started in one of the 'healthy' state, either with or without tamoxifen, depending upon the cohort they belonged to. Health states from 3 to 8 are disease states and are specifically included in the model because tamoxifen can potentially alter their risks significantly either in the positive or negative direction. The BCPT results suggest that tamoxifen will negatively affect (lower) risks of developing IBC and NIBC, and positively affect (increase) risk of developing EC, PE, DVT, and Cat-S. The 'Health' state has been separated into two distinct states, 'H-Tam' and 'H-noTam'. This is to discriminate those women who were healthy and consumed chemopreventive tamoxifen from those who were healthy but did not consume chemopreventive tamoxifen. These two states have different costs and event rates and consequently are represented as two distinct states. This structure also allows for the fact that a woman who develop tamoxifen-related adverse events such as PE, DVT or cataract surgery can return to healthy state with no tamoxifen (refer to assumption \# 4 of phase I in Chapter 3: women who develop any of the tamoxifen related adverse event stop consuming tamoxifen).

As is evident from the model, individuals from all states ultimately transitioned to 'Death' state. No arrow comes out of the 'Death' state (which means that once a woman transitions to the 'Death' state she remains in the 'Death' state forever). Thus the 'Death' state is termed as an absorbing state. This also means that in the due course of time, the proportion of women in the non-dead states will gradually diminish while it will increase in the 'Death' state. 
All women in the two tamoxifen intervention arms start in the "healthy with tamoxifen' state reflecting perfect initial health for all members of the cohort. Once the simulation begins, the initial cohort gets distributed into different health states including 'healthy with tamoxifen' and 'Death' depending upon the pre-specified transition probabilities. Women who survived each cycle (1 year) moved to the next cycle and were exposed to the risk of dying or going into one of the remaining health states. All transitions are assumed to occur instantaneously halfway through each cycle. However, some transitions were restricted in the model so as to make the model more manageable.

Once the women developed IBC, NIBC, EC they were not allowed to move into any of the two (with or without tamoxifen) healthy states. Moreover, health states such as IBC and NIBC were given priorities over other events excluding death. For instance, once a woman developed IBC, she stayed in that state or died due to IBC or other competing causes (all cause mortality). If the woman developed NIBC first, then she would stay in NIBC state or transit into IBC or Death state. Similarly, if a woman developed EC then she would either remain in the EC state or was allowed to transit into IBC, NIBC or Death state. Women in the remaining disease states such as PE, DVT, or cataract could remain in that states but were also allowed to transit to any remaining states excluding 'healthy with tamoxifen'. In other words, it was assumed that once a woman develops any of the adverse events associated with tamoxifen, she would be immediately taken off the tamoxifen therapy and thus transit into a healthy state with no tamoxifen (healthy with no tamoxifen state). For more information on a complete list of assumptions and their justification please refer to the next section 'Model Assumption and Rationale'.

Intuitively, the cohort simulation should terminate when all members of the cohort are dead (100\% of cohort in dead state). However, Markov processes in cohort simulations are evaluated probabilistically as if the cohort has infinite number of members (DATA TreeAge ${ }^{\mathrm{TM}}$ software manual). Thus, although more and more proportion of cohort members transit into 'Death' state over time, there is never a time when all the members are in the 'Death' state. In other words, the proportion of members 
in 'non-dead' states, although declines and approaching zero, is never equal to zero. Thus, an approximation has to be introduced to artificially terminate the cohort such that the approximation error is minimal. The simulation can be terminated when any error introduced due to 'pre-mature' termination of simulation is small compared to the totalpatient-cycles accumulated during the analysis (Beck J et al., 1983). The cohort in the present study was thus followed till $99.999 \%$ of cohort was in 'dead' state and the remainder was treated as an error of approximation. This is a typical feature of life expectancy models.

\section{Model Assumptions and Rationale}

1. Results from the National Surgical Adjuvant Breast and Bowel Project (NSABP) Breast Cancer Prevention Trial (BCPT) protocol P-1 (Fisher et al., 1998) are generalizable.

Rationale: The BCPT was a double-blind placebo-controlled multi-center trial involving 131 sites throughout United States and Canada. It enrolled 13,175 high-risk women with a total of 46,858 woman years of follow-up (Pritchard K, 1998). Hence, it was assumed that results from the BCPT were robust and generalizable.

2. Invasive breast cancer (IBC) took precedence over other health events.

Rationale: It was assumed in the model that once a woman develops invasive breast cancer, she could either remain in that state or die from invasive breast. No transition for invasive breast cancer to any other state, other than 'Death' was allowed. This was to avoid creation of multiple health states (for instance, IBC $+\mathrm{EC}$ health state, in which a women has both IBC and EC) which have very low probability of occurrence. For instance, based on results from BCPT trial, incidence rates for IBC in all women is 3.43 per 1,000 women in tamoxifen group and 6.76 per 1,000 women in placebo group (Fisher B et al., 1998). Incidence rates for EC were 2.3 per 1,000 in tamoxifen group and 0.91 per 1,000 women in placebo group. Probability of these two events occurring in the same woman (i.e. IBC+EC health state) will be about $8 *$ $10^{-6}$ in tamoxifen group and $6 * 10^{-6}$, extremely small likelihood as compared to likelihood of occurrence of other events considered in the model. 
3. The model assumes that once a woman develops IBC, NIBC, or EC she cannot transit into 'healthy' state.

Rationale: This assumption is based on the observation that although patients with $\mathrm{IBC}, \mathrm{NIBC}$ or EC can be in remission there is no cure for these cancers. Because IBC consumes more resources than EC and because no multiple states were allowed, it was assumed that patients with EC could transit to IBC but opposite was not true. Similarly as NIBC consumes more resources than EC, one way transition from EC to NIBC was allowed. On similar basis, as IBC consumed more resources than NIBC one-way transition from NIBC to IBC was allowed but the opposite was not true.

4. Tamoxifen therapy will be stopped as soon as a woman develops any of the tamoxifen related adverse events.

Rationale: This assumption in accordance with the clinic practice followed in the BCPT trial.

5. It was assumed that once tamoxifen therapy is stopped, subsequent tamoxifen-related excess risk of developing adverse events would cease as soon as the therapy is stopped. In other words, there will not be any 'spill-over' effect of tamoxifen. Rationale: Here the assumption is that there is no 'spill over' effect of tamoxifen. Although this may not be true in all cases, there is no way to determine the exact extent of this "spill-over" effect. This assumption was employed for both the 'beneficial' and 'harmful' effects of tamoxifen. Thus, if a woman ceases to be on tamoxifen therapy, the breast cancer risk reducing effects of tamoxifen would cease as soon as the therapy is stopped.

\section{Data for the Model}

The following section involves detailed description of data that was used to populate the model. Different types of data that were required to populate the model were: (1) risk (probabilities) of diseases, (2) all-cause and disease-specific mortality rates, and (3) disease treatment costs. 
Risk of diseases

Incidence rates for diseases were obtained from the NSABP's BCPT study. Table 3.1 illustrates average rates for women of all ages. However, in the model age-specific rates were employed.

Table 3.1: Incidence Rates based on the BCPT study*

\begin{tabular}{lcc}
\hline Disease & Tamoxifen Group & Placebo (Non-tamoxifen) Group \\
\hline Invasive breast cancer & $0.343 \%$ & $0.676 \%$ \\
Non-invasive breast cancer & $0.135 \%$ & $0.268 \%$ \\
Endometrial cancer & $0.230 \%$ & $0.091 \%$ \\
Pulmonary embolism & $0.069 \%$ & $0.023 \%$ \\
Deep vein thrombosis & $0.134 \%$ & $0.084 \%$ \\
Cataract surgery & $0.472 \%$ & $0.300 \%$ \\
\hline
\end{tabular}

* Average rates for women of all ages. However, age-specific rates from the BCPT were actually used in the model

$\underline{\text { All-cause and Disease-specific Mortality Rates }}$

All cause age- and sex-adjusted mortality were obtained from U.S. Bureau of Census (1991). Disease specific-mortality was obtained from other published sources. Mortality rates for IBC and DVT were obtained form Noe et al (1999). Mortality rates for NIBC and EC were obtained from American Cancer Society (American Cancer Society, 2000) and mortality rates for PE were obtained from a study by Carson et al (1992). As before, these rates were converted into probabilities using the density method and were then used in the model to determine the life expectancy (LE) of the cohort.

\section{Disease Treatment Costs}

The analysis was performed from WV Medicaid's perspective (payer's perspective) and hence only direct costs were incorporated in the analysis. Indirect costs, for instance, cost due to lost productivity, time lost from work, and non-medical costs 
were excluded. Direct medical costs included cost of chemopreventive tamoxifen, cost of mammography screening including follow-up costs resulting from abnormal mammogram report, and the cost of treating the major medical complications, which resulted from tamoxifen chemopreventive intervention as reported by the NSABP study. These complications include endometrial cancer, pulmonary thromboembolism, deep vein thrombosis, and cataract. Although, greater proportion of women in the tamoxifen group experienced hot flashes and vaginal discharge than in the placebo group, costs of treating such events were not included in the model as it is difficult to quantify costs for treating such events. Cost estimates of interventions and disease management were derived from literature and are presented in Table 3.2.

All costs were adjusted for increases in the medical care component of the Consumer Price Index and are given in 2001 dollars (U.S. Bureau of Labor Statistics). All costs and benefits will be discounted at a rate of $3 \%$ in the base case analysis. This is the discount rate accepted by Panel on Cost-Effectiveness in Health and Medicine, US Public Health Service (Gold M et al., 1996, Siegel J et al.,1996). Due to controversy surrounding the exact discount rate to be used in such studies, the model was separately run using $0 \%$ and $5 \%$ discount rates as a part of sensitivity analysis for the model. 
Table 3.2: Treatment and Drug Costs

\begin{tabular}{|c|c|c|}
\hline Item & Base-Case Cost* & Source \\
\hline \multicolumn{3}{|l|}{ Invasive Breast Cancer } \\
\hline Initial care cost & $\$ 16,122$ & Taplin et al., 1995 \\
\hline Continuing care cost & $\$ 1,603$ & \\
\hline Terminal care cost & $\$ 25,869$ & \\
\hline \multicolumn{3}{|l|}{ Non-Invasive Breast Cancer } \\
\hline Initial care cost & $\$ 12,219$ & Taplin et al., 1995 \\
\hline Continuing care cost & $\$ 1,274$ & \\
\hline Terminal care cost & $\$ 16,104$ & \\
\hline Endometrial Cancer & $\$ 5,649$ & Hershman D et al., 2001 \\
\hline Pulmonary Embolism & $\$ 9,634$ & Noe L et al., 1999 \\
\hline Deep Vein Thrombosis & $\$ 4,435$ & Noe L et al., 1999 \\
\hline Cataract Surgery & $\$ 3,488$ & Hershman D et al., 2001 \\
\hline \multirow[t]{2}{*}{ Tamoxifen 10 mg twice daily (annual cost) } & $\$ 1,221$ & WV Medicaid reimbursement rates \& \\
\hline & & Drug Topics Red Book, 1999 \\
\hline Mammography Screening (including follow-up) & $\$ 100$ & Noe L et al., 1999 \\
\hline
\end{tabular}

* All figures adjusted to 2001 dollars and rounded to the nearest whole number 
The usual course of most cancers involves intensive therapy, which usually involves surgery and chemotherapy immediately following diagnosis. This initial phase is then followed by period when little cancer-related medical care occurs other than monitoring for recurrence or metastases. Finally, the intensity of medical care increases significantly prior to death of the cancer patient. Since this pattern of medical care utilization is relatively consistent with most cancers, including breast cancer, Taplin et al., (1995) divided the treatment costs of the patients into three distinct phases (1) initial phase, (2) intermediate phase, and (3) terminal phase. This approach has widely been used for calculating cancer related treatment costs (Riley $\mathrm{G}$ et al). Hence, the weighted costs of treating invasive and non-invasive breast cancer were calculated based on estimates obtained from Taplin et al., (1995) study. Thus, in the current model, patients experience higher costs in the first six months after they develop breast cancer and six months before they die due to breast cancer. These breast cancer patients continue to experience the 'continuing care cost' when they are in the breast cancer state of the model.

The endometrial cancer costs and cataract surgery costs were obtained from published literature (Hershman D et al., 2001). These costs were based on Medicare payments for 1998 from the Centers for Medicare and Medicaid (CMS) (previously known as the Health Care Financing Administration (HCFA)). The pulmonary embolism cost and deep vein thrombosis costs were obtained from a study by Noe and colleagues (1999). Tamoxifen costs were based on 1999 Red Book prices. The WV Medicaid reimbursement formula (AWP $* 88 \%+\$ 3.90$ ) per prescription was used to calculate the annual drug costs. The mammography screening costs were obtained from a study by Salzmann P et al., (1997). The screening costs included cost of mammography examination and cost of evaluating subsequent abnormal mammograms. The mammography examination cost was based on Medicare reimbursement fee for screening. In the Salzmann et al., study, the incremental cost due to abnormal mammograms were calculated as a weighted average of procedures that followed abnormal mammograms based on report by the National Cancer Institute's National Survey of Mammography Facilities (Brown et al., 1995). 


\section{Phase II}

Data for phase II of the study will be collected using a mail survey. This survey was developed in order to assess the respondents' individual risk of developing breast cancer, their perceived risk of breast cancer, their current usage and inclination for using breast cancer preventive strategies, and knowledge about these strategies.

\section{Defining the Study Population}

Population is an aggregation of study elements (Babbie, 1989). In most cases, it is practically impossible to survey the entire population. In such cases, a statistically desirable proportion of this population has to be studied. This proportion is called the 'study sample.'

Results obtained from studying the 'sample' can then be extrapolated to the entire population. The population for Phase I of this study consists of all white women 40 years of age and above who are enrollees of WV Medicaid enrollees.

\section{Sampling Technique}

A study sample was drawn from the sampling frame using Simple Random Sampling (SRS) without replacement technique (Kalton G, 1987). In this technique, the randomly selected elements in the sample are not replaced to be given further chance of selection. As SRS without replacement gives more precise estimators than sampling with replacement, this technique was used in creating the sample.

Objectives of Phase II require either mean (for instance, knowledge scores) or proportion (for instance, proportion of high-risk women) estimations. SRS technique allows estimation of both means as well as proportion along with the associated confidence interval (Kalton G, 1987).

\section{Study Sample \& Sample Size Determination}

All white women aged 40 years and above, who were West Virginia Medicaid enrollees in 1999 (the last year of available data) were included for study sample 
determination for phase I. The year 1999 was selected in order to utilize the latest available enrollment data in order to increase the likelihood that study subjects surveyed in 2002 will still be WVMP enrollees. The survey questionnaire will be administered to randomly selected Medicaid women enrollees satisfying the study inclusion criteria.

As SRS is employed for sampling, the following formula will be used in order to determine appropriate sample size (Kalton G, 1987).

$$
\mathrm{n}=(\mathrm{z} / \mathrm{e})^{2} * \pi(1-\pi)
$$

where,

$\mathrm{n}$ is the size of the sample

$\mathrm{z}$ is the number of standard errors for given confidence interval

$\pi$ is the estimated proportion of people at high risk of developing breast cancer

$\mathrm{e}$ is the required estimator

Based on table of normal distribution (95\% of normal distribution falls within 1.96 standard deviations around the distribution's means), for confidence level of $95 \%, \mathrm{z}$ $=1.96$. An estimator that is within $5 \%$ of the population percentage with $95 \%$ probability will be employed in the study (Kalton G, 1987).

In the NSABP's BCPT trial, risk assessments were performed for 98,018 women out of whom 57,641 were deemed to be at high risk of developing breast cancer (Fisher et al., 1998). In other words, a total of about $59 \%$ were in high-risk group. Thus, in the above equation ' $\pi$ ' should assume a value of 0.59 . However, in the present context, a conservative approach was taken for sample size calculation by assuming a value of 0.50 for ' $\pi$ ' yielding a higher required sample size.

Inputting values just discussed into the above equation yielded $\mathrm{N} \cong 384$.

Net Sample Size $=($ Gross Sample Size $) *($ Mail Response Rate $) *($ Usable Response Rate) 
Hence, Gross Sample Size = Net Sample Size / (Mail Response Rate) * (Usable Response Rate)

Based on studies involving Medicaid population, we assumed a conservative response rate of $20 \%$ for mail survey. Moreover, we assumed that about $90 \%$ of responses will be usable. Hence,

$$
\text { Gross sample size }=384 / 0.20 * 0.9 \cong 2000
$$

Thus, a total of 2000 Medicaid women recipients were surveyed.

\section{$\underline{\text { Instrument Development }}$}

The questionnaire used for phase I of the study was a structured-undisguised survey (Seltiz C, et al.,), whereby the purpose of the study was disclosed to the respondents by means of a cover letter enclosed with the questionnaire. A detailed description of the survey questionnaire is provided in the section titled 'Survey Questionnaire'.

\section{Survey Questionnaire}

The survey questionnaire was designed to gather information on following components of breast cancer prevention management: (1) current utilization of breast cancer preventive strategies, (2) perceived risk of developing breast cancer, (3) actual risk of developing breast cancer as calculated by Gail model, (4) breast cancer and breast cancer prevention knowledge, and (5) willingness to use breast cancer preventive strategies.

For assessing utilization, the surveyed women were asked about their mammography screening status over a period of time. Utilization of chemopreventive tamoxifen was assessed in a similar manner. The next set of questions gathered information on woman's perceived risk of developing breast cancer. The women were asked to estimate their probability of developing breast cancer in next 5 years, and over their entire lifetime. This perceived probability was then compared to actual probabilities 
of developing breast cancer as calculated using Gail Model (Gail et al., 1989). These perceptions were measured across various age- and risk-groups.

Woman's actual risk of developing breast cancer was assessed next. This included obtaining information on woman's age, age at menarche, age at first live birth, number of previous biopsies, number of first degree relatives with breast cancer, and presence of atypical hyperplasia (AH). This information was used in breast cancer risk assessment. Gail model for breast cancer risk assessment was employed to do the same. Relative risks calculation are based on an unconditional logistic regression that included main effects of age at menarche (AGEMEN), number of previous breast biopsies (NBIOPS), age at first live birth (AGEFLB), and number of first degree relatives with breast cancer (NUMREL) (Gail et al., 1989). Also included in the equation are interaction effects between AGECAT and NBIOPS and between AGEFLB and NUMREL. The final logistic regression equation on basis of which the relative risks are calculated is:

$\ln ($ relative risk $)=-0.74948+0.09401($ AGEMEN $)+0.52926(\mathrm{NBIOPS})+$ $0.21863(\mathrm{AGEFLB})+0.95830(\mathrm{NUMREL})+0.01081(\mathrm{AGECAT})-$ 0.28804(NBIOPS*AGECAT) + 0.19081(AGEFLB*NUMREL)

The above mentioned risk factors are coded either as 0,1 or 2 depending upon status of risk factors. For example, for a woman with no history of biopsy, the variable NBIOPS was coded as 0 . On the other hand if the women has undergone biopsy once or more than once then variable NBIOPS are coded as 1 or 2 , respectively, as the case may be.

Women's breast cancer and breast cancer prevention knowledge was measured next. They were asked to choose one of the options from the response set of "Agree", “Don't Agree", or "Don't Know" on questionnaire items such as "breast cancer risk increases with increasing age" and "family history of breast cancer increases the personal risk of getting breast cancer." 
Questionnaire Validity

Faculty members from School of Pharmacy and Department of Community Medicine, and individuals from the West Virginia Medicaid Bureau provided comments on questionnaire, which were incorporated in order to enhance the content validity of the questionnaire. Some of the aspects for assessing content validity included relevance of questions, language understanding, and instruction clarity. Other aspects of testing questionnaire included assessment of ease of reading, and readability levels of the respondents.

\section{Instrument Administration}

Information needed to accomplish phase I was obtained by means of a mail survey. The survey process was initiated with an introductory letter to randomly selected WV Medicaid enrollees informing them about the study and the possibility that they might receive a survey regarding the study. This introductory mailing was followed by a total of three survey mailings in order to obtain sufficient responses to the survey. A non-response survey was sent to a random sample of those individuals who did not respond to any of the three mailings.

Data was collected over a period of two months from August to September 2002. Approvals for all survey related documents were sought from both the West Virginia Medicaid Bureau (WVMB) and the West Virginia University's Institutional Review Board (WVU-IRB). Once the documents were approved by the WVMB, they were sent to WVU-IRB for an expedited review and final approval.

An initial letter from the WV Medicaid Commissioner, informing the survey recipients about the study, was mailed about four days prior to the first questionnaire. The questionnaire was mailed along with a cover letter signed by the WV Medicaid Commissioner. The cover letter informed the recipients of the intent of the survey and the importance of recipients to respond to the survey. The cover letter also emphasized that participation in the survey was purely voluntary, assured them confidentiality, and indicated that their services will not be affected by their participation or non- 
participation. A second mailing of the survey was sent to non-respondents two weeks after the first survey. Non-respondents to the second survey were mailed a third survey. Last, a non-response survey was mailed to those not responding to any of the three surveys. The non-response survey collected information on breast cancer risk factors and also reasons for non-participation.

\section{$\underline{\text { Survey Assumptions }}$}

1. Women who responded to the survey were similar to the WV Medicaid women population in terms of their demographics and breast cancer risk characteristics Rationale: The survey questionnaire did not gather information on demographics characteristics such as the socio-economic status as it was specifically designed to estimate breast cancer risk and other aspects of breast cancer prevention management. Moreover, some of the breast cancer risk information such as age of menarche that was gathered in the survey is not usually available in any Medicaid data. Hence, comparison of the respondents to the WV Medicaid women population was not possible. However, the survey 'sample' was created based on the simple random sampling technique, which ensures that each member of the population has equal probability of being selected in the sample, thereby increasing the likelihood that the 'sample' is similar to the population from which it was created.

2. Self-reported data and associated recall bias

Rationale: As most of the information required to estimate a woman's risk of developing breast cancer is not readily available in secondary databases and records, significant amount of information for phase II of this study was self-reported. The assumption in the study was that the recall bias was minimal. A 'Don't know' response option was provided in the survey questionnaire for majority of the questions so that the respondents could mark that in case they did not know or recollect the information asked. 


\section{CHAPTER 4}

\section{RESULTS}

\section{Phase I}

Phase I involved the development of a Markov process that simulated three hypothetical cohorts of women who were otherwise healthy but were at a high risk of developing breast cancer. Women in the first cohort started consuming chemopreventive tamoxifen at age 40 and continued to do so till they were 45 years old and had routine mammography screening in accordance with the American Cancer Society's (ACS) breast cancer screening guidelines. Women in the second cohort started consuming chemopreventive tamoxifen at age 40 and continued to do so till they were 45 years old and underwent mammography screening at rates that were observed in the real-world setting. Women in the third cohort also entered the model at the age 40 but did not consume chemopreventive tamoxifen but underwent mammography screening at rates that were observed in the real-world setting.

The following list of tables present the transition probabilities between different health states. These transition probabilities dictate the distribution of cohorts among different states at the end of each Markov cycle. As many of the transition probabilities are time-dependent, it is not possible to report them in the same table. Table 4.1 lists transition probabilities that are time-independent (remain constant and do not vary with time) and provides reference table numbers for other transition probabilities that are timedependent. 
Table 4.1: Transition Probability Matrix

\begin{tabular}{|c|c|c|c|c|c|c|c|c|c|}
\hline Health State & H-Tam & H-noTam & IBC & NIBC & $\mathrm{EC}$ & $\mathrm{PE}$ & DVT & Cat-S & Death \\
\hline H-Tam & $*$ & 0.0654 & Tab 4.2 & Tab 4.2 & Tab 4.2 & Tab 4.2 & Tab 4.2 & Tab 4.2 & $f(\mathrm{ASR})$ \\
\hline H-noTam & 0.0000 & $*$ & Tab 4.3 & Tab 4.3 & Tab 4.3 & Tab 4.3 & Tab 4.3 & Tab 4.3 & $f(\mathrm{ASR})$ \\
\hline IBC & 0.0000 & 0.0000 & $*$ & 0.0000 & 0.0000 & 0.0000 & 0.0000 & 0.0000 & $f(\mathrm{ASR}+\mathrm{IBC})$ \\
\hline NIBC & 0.0000 & 0.0000 & 0.0069 & * & 0.0000 & 0.0000 & 0.0000 & 0.0000 & $f(\mathrm{ASR}+\mathrm{NIBC})$ \\
\hline $\mathrm{EC}$ & 0.0000 & 0.0000 & 0.0067 & 0.0027 & $*$ & 0.0000 & 0.0000 & 0.0000 & $f(\mathrm{ASR}+\mathrm{EC})$ \\
\hline PE & 0.0000 & 0.8000 & 0.0067 & 0.0027 & 0.0011 & $*$ & 0.0000 & 0.0000 & $f(\mathrm{ASR}+\mathrm{PE})$ \\
\hline DVT & 0.0000 & 0.8000 & 0.0067 & 0.0027 & 0.0011 & 0.0000 & $*$ & 0.0000 & $f(\mathrm{ASR}+\mathrm{DVT})$ \\
\hline Cat-S & 0.0000 & $*$ & 0.0067 & 0.0027 & 0.0011 & $9.9 * 10^{-5}$ & 0.0008 & 0.0000 & $f(\mathrm{ASR}+\mathrm{Cat}-\mathrm{S})$ \\
\hline Death & 0.000 & 0.000 & 0.000 & 0.000 & 0.000 & 0.000 & 0.000 & 0.000 & 1.000 \\
\hline \\
\hline \multicolumn{10}{|c|}{$f($ ASR + IBC): Function of age-sex-race adjusted all cause mortality + IBC specific mortality } \\
\hline \multicolumn{10}{|c|}{$f($ ASR + NIBC): Function of age-sex-race adjusted all cause mortality + NIBC specific mortality } \\
\hline \multicolumn{10}{|c|}{$f($ ASR + EC): Function of age-sex-race adjusted all cause mortality + EC specific mortality } \\
\hline \multicolumn{10}{|c|}{$f(\mathrm{ASR}+\mathrm{PE}):$ Function of age-sex-race adjusted all cause mortality + PE specific mortality } \\
\hline \multicolumn{10}{|c|}{$f(\mathrm{ASR}+\mathrm{DVT})$ : Function of age-sex-race adjusted all cause mortality + DVT specific mortality } \\
\hline
\end{tabular}


Non-compliance with chemopreventive tamoxifen was incorporated in the model by allowing direct transition between the 'H-Tam' to 'H-nonTam' health state. Based on BCPT, non-compliance was estimated to be $23.7 \%$ over a four year period (the mean follow-up time for the BCPT was 47.7 months which is about 4 years) (Fisher B et al., 1998). The 'density method' was employed to determine 'risk' or 'probability' over the time interval (Miller D et al., 1994) in the following manner

$$
\mathrm{P}_{\text {non-compliance }}=1-[1-0.237]^{1 / 4}=0.0654
$$

Age-dependent rates of transition from the 'H-Tam' state to other states were obtained from the BCPT. The density method was used to convert these rates into transition probabilities. The calculated transition probabilities are reported in Table 4.2. The beneficial and adverse events of chemopreventive tamoxifen taken for 5 years were assumed to last for the time period over which it was consumed i.e. 5 years. Thus, transitions between 'H-Tam' state to other states become equivalent to those observed in the non-tamoxifen group after 5-year time interval. 
Table 4.2: Age-dependent probabilities [H-Tam to other health states]

\begin{tabular}{lr}
\hline Health State/Age (years) & Transition Prob \\
\hline From H-Tam to IBC & 0.00376 \\
$40-44$ & 0.00668 \\
$45-49$ & 0.00626 \\
$50-59$ & 0.00730
\end{tabular}

From H-Tam to NIBC

$40-44$

0.00135

45-above

0.00268

From H-Tam to EC

$40-44$

0.00132

45-49

0.00109

50-above

0.00076

From H-Tam to PE

$40-44$

0.00019

45-49

0.00009

50-above

0.00031

From H-Tam to DVT

$40-44$

0.0011

45-49

0.00078

50-above

0.00088

From H-Tam to Cat-S

$40-44$

0.00471

45-above

0.00299

\section{From H-Tam to Death}

40 - above

Age-Sex-Race adjusted mortality (US Census)

H-Tam: Healthy with Tamoxifen, H-noTam: Healthy with no Tamoxifen IBC: Invasive breast cancer, NIBC: Non-invasive breast cancer, EC: Endometrial cancer PE: Pulmonary embolism, DVT: Deep vein thrombosis, Cat-S: Cataract surgery 
Table 4.3 presents transition probabilities from the 'H-noTam' group to other health states in the model. A comparison of probability estimates in Table 4.3 and Table 4.2 will reveal that transition probabilities after the first 5 years of cohort simulation assume same values. It accounts for the fact that the effect (both beneficial and adverse) of chemopreventive therapy with tamoxifen ceases after 5 years.

Once a woman develops invasive breast cancer, it was assumed that she remains in the 'IBC' state or dies, either from IBC or non-IBC related cause. Hence, no transitions were allowed from IBC to any other health state except the 'Death' state. In real life, it is possible for a woman with breast cancer to move to any of the above health states. However, in modeling terms it means creating more states, for instance, IBC + EC wherein a woman has both IBC and EC or even IBC + EC + PE wherein a woman develops all three conditions. This makes the model very complex and unmanageable. Justification for this assumption is provided in phase I assumption section in Chapter 3 (assumption \# 2).

Similar assumptions were applied to transitions from 'NIBC' to other states. An exception here was that an additional transition was allowed from 'NIBC' to 'IBC'. In fact, the transition probability from 'NIBC' to 'IBC' was adjusted higher than general transition probability (to 'IBC' from other states) based on the published literature indicating higher rates of NIBC progressing to become IBC. Women who develop EC could either transition to 'IBC' or 'NIBC', remain in 'EC', or die due to EC or non-EC related cause. These assumptions regarding transitions were relaxed for 'PE', 'DVT' and 'Cat-S' in the sense that women who develop any of these conditions could transit back to healthy state, 'H-noTam.' For 'Cat-S', all transitions were possible except that a woman could remain in the 'Cat-S' state for no more than one year (one cycle) as cataract surgery is an acute event and does not extend beyond one year or in this case one cycle. 
Table 4.3: Age-dependent probabilities [H-noTam to other health states]

Health State/Age (years) Transition Probabilities

\section{From H-noTam to IBC}

$40-44$

0.00668

$45-49$

0.00668

$50-59$

0.00626

60- above

0.00730

From H-noTam to NIBC

40 -above

0.00268

From H-noTam to EC

$40-49$

0.00109

50-above

0.00076

\section{From H-noTam to PE}

$40-49$

0.00009

50-above

0.00031

\section{From H-noTam to DVT}

$40-49$

0.00078

50-above

0.00088

\section{From H-noTam to Cat-S}

40 -above

0.00299

\section{From H-noTam to Death}

40 - above

Age-Sex-Race adjusted mortality (US Census)

H-Tam: Healthy with Tamoxifen, H-noTam: Healthy with no Tamoxifen IBC: Invasive breast cancer, NIBC: Non-invasive breast cancer, EC: Endometrial cancer PE: Pulmonary embolism, DVT: Deep vein thrombosis, Cat-S: Cataract surgery 
Transition from individual health states to 'Death' can be considered as a function of two independent forces: $f\left(\mathrm{TP}_{\mathrm{dsm}}+\mathrm{TP}_{\mathrm{m}}\right)$, where $\mathrm{TP}_{\mathrm{dsm}}$ is disease specific mortality and $\mathrm{TP}_{\mathrm{m}}$ is all-cause mortality excluding the disease under consideration. This function reflects the fact that an individual in a particular disease state may either die due to that disease $\left(\mathrm{TP}_{\mathrm{dsm}}\right)$ or may die due to non-disease specific cause $\left(\mathrm{TP}_{\mathrm{m}}\right) . \mathrm{TP}_{\mathrm{m}}$ is also referred to as all-cause mortality. Age-Sex-Race (ASR) adjusted all-cause mortality rates were obtained from Statistical Abstract of the United States (U.S. Census Bureau (Vital Statistics), 1998) to simulate age-dependent mortality rates among white women. These rates were then converted into probabilities using the density method. Mortality transition probabilities for each of the health states, except cataract (Cat-S), are reported in the following tables (Table 4.4 to Table 4.8). Mortality from cataract surgery was assumed to be 'zero'. However, women with cataract surgery could still die from allcause mortality in the model. 
Table 4.4: Invasive Breast Cancer (IBC)-adjusted mortality rates

\begin{tabular}{cccccccccc}
\hline Age & Mortality & Age & Mortality & Age & Mortality & Age & Mortality & Age & Mortality \\
\hline 40 & 0.03323 & 50 & 0.03497 & 60 & 0.03990 & 70 & 0.05094 & 80 & 0.07938 \\
41 & 0.03332 & 51 & 0.03530 & 61 & 0.04070 & 71 & 0.05094 & 81 & 0.07938 \\
42 & 0.03342 & 52 & 0.03565 & 62 & 0.04154 & 72 & 0.05094 & 82 & 0.07938 \\
43 & 0.03354 & 53 & 0.03601 & 63 & 0.04239 & 73 & 0.05094 & 83 & 0.07938 \\
44 & 0.03367 & 54 & 0.03640 & 64 & 0.04328 & 74 & 0.05094 & 84 & 0.07938 \\
45 & 0.03381 & 55 & 0.03683 & 65 & 0.04423 & 75 & 0.06166 & 85 & 0.65203 \\
46 & 0.03398 & 56 & 0.03730 & 66 & 0.04423 & 76 & 0.06166 & 86 & 0.65203 \\
47 & 0.03417 & 57 & 0.03784 & 67 & 0.04423 & 77 & 0.06166 & 87 & 0.65203 \\
48 & 0.03440 & 58 & 0.03845 & 68 & 0.04423 & 78 & 0.06166 & 88 & 0.65203 \\
49 & 0.03467 & 59 & 0.03914 & 69 & 0.04423 & 79 & 0.06166 & $\geq 89$ & 0.65203 \\
\hline
\end{tabular}


Table 4.5: Non-Invasive Breast Cancer (NIBC)-adjusted mortality rates

\begin{tabular}{cccccccccc}
\hline Age & Mortality & Age & Mortality & Age & Mortality & Age & Mortality & Age & Mortality \\
\hline 40 & 0.00328 & 50 & 0.00505 & 60 & 0.01008 & 70 & 0.02133 & 80 & 0.05032 \\
41 & 0.00337 & 51 & 0.00538 & 61 & 0.01089 & 71 & 0.02133 & 81 & 0.05032 \\
42 & 0.00347 & 52 & 0.00574 & 62 & 0.01174 & 72 & 0.02133 & 82 & 0.05032 \\
43 & 0.00359 & 53 & 0.00611 & 63 & 0.01261 & 73 & 0.02133 & 83 & 0.05032 \\
44 & 0.00372 & 54 & 0.00651 & 64 & 0.01352 & 74 & 0.02133 & 84 & 0.05032 \\
45 & 0.00387 & 55 & 0.00695 & 65 & 0.01449 & 75 & 0.03226 & 85 & 0.63412 \\
46 & 0.00404 & 56 & 0.00743 & 66 & 0.01449 & 76 & 0.03226 & 86 & 0.63412 \\
47 & 0.00424 & 57 & 0.00797 & 67 & 0.01449 & 77 & 0.03226 & 87 & 0.63412 \\
48 & 0.00447 & 58 & 0.00860 & 68 & 0.01449 & 78 & 0.03226 & 88 & 0.63412 \\
49 & 0.00475 & 59 & 0.00930 & 69 & 0.01449 & 79 & 0.03226 & $\geq 89$ & 0.63412 \\
\hline
\end{tabular}


Table 4.6: Endometrial Cancer (EC)-adjusted mortality rates

\begin{tabular}{cccccccccc}
\hline Age & Mortality & Age & Mortality & Age & Mortality & Age & Mortality & Age & Mortality \\
\hline 40 & 0.03555 & 50 & 0.03732 & 60 & 0.04235 & 70 & 0.05360 & 80 & 0.08259 \\
41 & 0.03564 & 51 & 0.03765 & 61 & 0.04316 & 71 & 0.05360 & 81 & 0.08259 \\
42 & 0.03574 & 52 & 0.03801 & 62 & 0.04401 & 72 & 0.05360 & 82 & 0.08259 \\
43 & 0.03586 & 53 & 0.03838 & 63 & 0.04488 & 73 & 0.05360 & 83 & 0.08259 \\
44 & 0.03599 & 54 & 0.03878 & 64 & 0.04579 & 74 & 0.05360 & 84 & 0.08259 \\
45 & 0.03614 & 55 & 0.03922 & 65 & 0.04676 & 75 & 0.06453 & 85 & 0.66639 \\
46 & 0.03631 & 56 & 0.03970 & 66 & 0.04676 & 76 & 0.06453 & 86 & 0.66639 \\
47 & 0.03651 & 57 & 0.04024 & 67 & 0.04676 & 77 & 0.06453 & 87 & 0.66639 \\
48 & 0.03674 & 58 & 0.04087 & 68 & 0.04676 & 78 & 0.06453 & 88 & 0.66639 \\
49 & 0.03702 & 59 & 0.04157 & 69 & 0.04676 & 79 & 0.06453 & $\geq 89$ & 0.66639 \\
\hline
\end{tabular}


Table 4.7: Pulmonary Embolism (PE)-adjusted mortality rates

\begin{tabular}{cccccccccc}
\hline Age & Mortality & Age & Mortality & Age & Mortality & Age & Mortality & Age & Mortality \\
\hline 40 & 0.02628 & 50 & 0.00305 & 60 & 0.00808 & 70 & 0.01933 & 80 & 0.04832 \\
41 & 0.00137 & 51 & 0.00338 & 61 & 0.00889 & 71 & 0.01933 & 81 & 0.04832 \\
42 & 0.00147 & 52 & 0.00374 & 62 & 0.00974 & 72 & 0.01933 & 82 & 0.04832 \\
43 & 0.00159 & 53 & 0.00411 & 63 & 0.01061 & 73 & 0.01933 & 83 & 0.04832 \\
44 & 0.00172 & 54 & 0.00451 & 64 & 0.01152 & 74 & 0.01933 & 84 & 0.04832 \\
45 & 0.00187 & 55 & 0.00495 & 65 & 0.01249 & 75 & 0.03026 & 85 & 0.63212 \\
46 & 0.00204 & 56 & 0.00543 & 66 & 0.01249 & 76 & 0.03026 & 86 & 0.63212 \\
47 & 0.00224 & 57 & 0.00597 & 67 & 0.01249 & 77 & 0.03026 & 87 & 0.63212 \\
48 & 0.00247 & 58 & 0.00660 & 68 & 0.01249 & 78 & 0.03026 & 88 & 0.63212 \\
49 & 0.00275 & 59 & 0.00730 & 69 & 0.01249 & 79 & 0.03026 & $\geq 89$ & 0.63212 \\
\hline
\end{tabular}


Table 4.8: Deep Vein Thrombosis (DVT)-adjusted mortality rates

\begin{tabular}{cccccccccc}
\hline Age & Mortality & Age & Mortality & Age & Mortality & Age & Mortality & Age & Mortality \\
\hline 40 & 0.00628 & 50 & 0.00305 & 60 & 0.00808 & 70 & 0.01933 & 80 & 0.04832 \\
41 & 0.00137 & 51 & 0.00338 & 61 & 0.00889 & 71 & 0.01933 & 81 & 0.04832 \\
42 & 0.00147 & 52 & 0.00374 & 62 & 0.00974 & 72 & 0.01933 & 82 & 0.04832 \\
43 & 0.00159 & 53 & 0.00411 & 63 & 0.01061 & 73 & 0.01933 & 83 & 0.04832 \\
44 & 0.00172 & 54 & 0.00451 & 64 & 0.01152 & 74 & 0.01933 & 84 & 0.04832 \\
45 & 0.00187 & 55 & 0.00495 & 65 & 0.01249 & 75 & 0.03026 & 85 & 0.63212 \\
46 & 0.00204 & 56 & 0.00543 & 66 & 0.01249 & 76 & 0.03026 & 86 & 0.63212 \\
47 & 0.00224 & 57 & 0.00597 & 67 & 0.01249 & 77 & 0.03026 & 87 & 0.63212 \\
48 & 0.00247 & 58 & 0.00660 & 68 & 0.01249 & 78 & 0.03026 & 88 & 0.63212 \\
49 & 0.00275 & 59 & 0.00730 & 69 & 0.01249 & 79 & 0.03026 & $\geq 89$ & 0.63212 \\
\hline
\end{tabular}


Base-case Analysis Results

A $3 \%$ discount rate was applied to both, benefits as well as costs, in the base case analysis. The $3 \%$ discount rate was employed based on recommendation of the "Panel on Cost-effectiveness in Health and Medicine" which was convened by the United States Public Health Service (Gold M et al., 1996). This discount rate reflects the current available data on real economic growth and corresponding estimates of the real consumption rate of interest according to the panel.

Full mammography compliance was assumed in the 'Tam + Optimal Mam Screen' cohort since one of the goals of the model was to estimate the incremental costs and benefits of chemopreventive tamoxifen in presence of mammography screening under optimal utilization criteria. In the 'Tam + Non-optimal Mam Screen' cohort, the mammography screening compliance was assumed to be $46.7 \%$ (Centers for Medicare and Medicaid (CMS), 1999). This estimate was based on mammography screening rates reported for the West Virginia Medicare beneficiaries who were enrolled in Medicaid. Although this population is different than the women population under consideration in terms of age and socioeconomic status, the mammography screening rates were the best available large-scale estimates and hence were used in the model. Moreover, a one way sensitivity analysis was performed on mammography rates to account for differences in screening rates among the two populations. The same screening compliance of $46.7 \%$ was applied to the 'NoTam + Non-optimal Mam Screen' cohort.

Table 4.9 reports the distribution of patient life-years among different health states and total life expectancy for the three cohorts based on model simulation. As indicated in Table 4.9, the average life expectancy of cohort in the 'Tam + Optimal Mam Screen' group was 21.585 years as compared to 21.539 years in the 'Tam + Non-optimal Mam Screen' cohort and 21.463 years in the 'NoTam + Non-optimal Mam Screen' cohort. This amounts to an incremental life expectancy gain of 0.122 years (or 44.53 days) in the 'Tam + Optimal Mam Screen' cohort and 0.076 years (or 27.74 days) in the 'Tam + Non-optimal Mam Screen' as compared to the 'NoTam + Non-optimal Mam Screen' cohort. 
Table 4.9: Simulated Cohort Life-Years* Distribution - Base-case

\begin{tabular}{lccc}
\hline Health State & $\begin{array}{c}\text { Tam + Optimal Mam } \\
\text { Screen (years) }\end{array}$ & $\begin{array}{c}\text { Tam + Non-optimal Mam } \\
\text { Screen (years) }\end{array}$ & $\begin{array}{c}\text { NoTam + Non-optimal } \\
\text { Mam Screen (years) }\end{array}$ \\
\hline H-Tam & 13.137 & 13.137 & N/A \\
H-noTam & 5.879 & 5.879 & 18.724 \\
IBC & 1.557 & 1.511 & 1.663 \\
NIBC & 0.714 & 0.714 & 0.804 \\
EC & 0.205 & 0.205 & 0.191 \\
PE & 0.006 & 0.006 & 0.005 \\
DVT & 0.021 & 0.021 & 0.019 \\
Cat-S & 0.066 & 0.066 & 0.057 \\
Death & 0.000 & 0.000 & 0.000 \\
Total & 21.585 years & 21.539 years & 21.463 years \\
\hline
\end{tabular}

* $3 \%$ discount rate

H-Tam: Healthy with Tamoxifen, H-noTam: Healthy with no Tamoxifen, IBC: Invasive breast cancer, NIBC: Non-invasive breast cancer, EC: Endometrial cancer, PE: Pulmonary embolism, DVT: Deep vein thrombosis, Cat-S: Cataract surgery

Patients in the non-tamoxifen cohort spent more time in the 'IBC' and 'NIBC' disease states (2.47 years in the 'non-tamoxifen' cohort versus 2.27 years in the 'Tam + Optimal Mam Screen' cohort and 2.23 years in the 'Tam + Non-optimal Mam Screen' cohort) while patients in the tamoxifen cohorts spent more time in the non-breast cancer disease states ( 0.29 years in the 'Tam + Optimal Mam Screen' and 'Tam + Non-optimal Mam Screen' cohorts versus 0.27 years in the 'NoTam + Non-optimal Mam Screen' cohort).

These results are consistent with the fact that tamoxifen results in lowering risk of IBC and NIBC while increasing risk of other disease conditions that are in the model. Obviously, years spent is the 'Death' state cannot be counted as life years and so 'zero' years are indicated in the table for 'Death' state, irrespective of the amount of time that a cohort spent in that state. 
These comparisons are shown in Figure 4.1 which is a graphical illustration of simulated life years distribution among three broad combined health states: Healthy (HTam and H-noTam), Breast Cancer (IBC+NIBC) state, and Non-breast cancer state $(\mathrm{EC}+\mathrm{PE}+\mathrm{DVT}+\mathrm{Cat}-\mathrm{S})$.

Figure 4.1: Simulated Cohort Life-Years Distribution for Combined States- Base-case

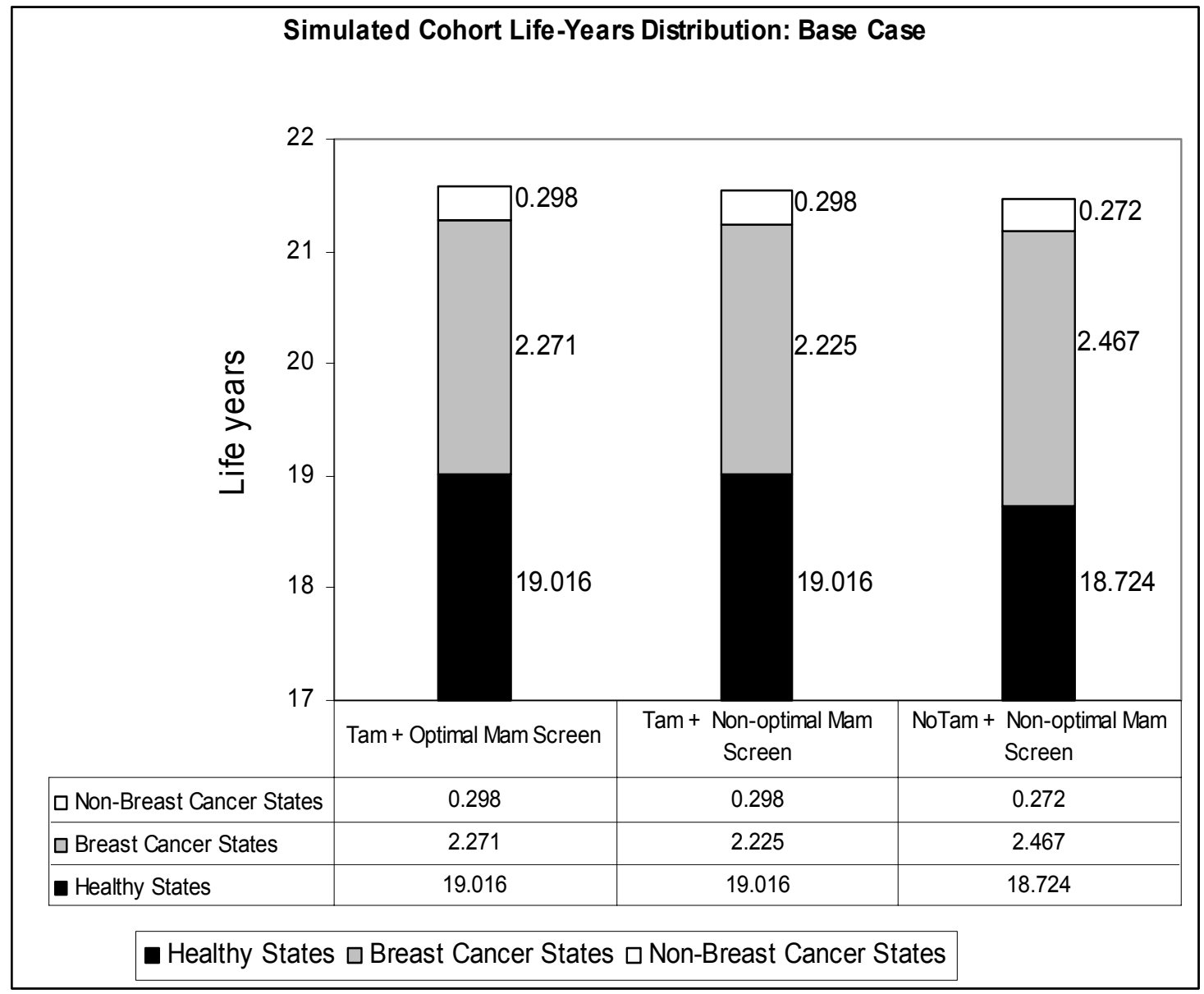

Magnitude of the costs within each disease state not only depends upon the cost of treating that disease condition but also on the amount of time spent by an individual in that disease state. Table 4.10 reports distribution of costs among different health states and the total costs for the three cohorts. For instance, due to breast cancer risk reduction attribute of chemopreventive tamoxifen, less average time is spent in the breast cancer states (both IBC and NIBC) by individuals in the 'tamoxifen' cohorts as compared to 
individuals in the 'non-tamoxifen' cohort (refer to Table 4.9). Hence, breast cancer costs (IBC + NIBC + Death; cost of the 'Death' state is included because costs in 'Death' state incur as a result of 'terminal' breast cancer treatment) are lower in the 'Tam + Optimal Mam Screen' cohort $(\$ 9,502.22)$ and 'Tam + Non-optimal Mam Screen' cohort $(\$ 9,467.79)$ as compared to 'NoTam + Non-optimal Mam Screen' cohort $(\$ 10,281.85)$ (Table 4.10). As chemopreventive tamoxifen increases risk of other disease conditions (EC, PE, DVT, and Cat-S), costs attributable to these conditions are higher in the 'Tam + Optimal Mam Screen' cohort $(\$ 1,567.48)$ and 'Tam + Non-optimal Mam Screen' cohort $(\$ 1,551.76)$ as compared to 'no-tamoxifen' group $(\$ 1,427.06)$.

Table 4.10: Simulated Cohort Cost* Distribution - Base-case

\begin{tabular}{lccc}
\hline \multicolumn{1}{c}{$\begin{array}{c}\text { Health } \\
\text { State }\end{array}$} & $\begin{array}{c}\text { Tam + Optimal Mam } \\
\text { Screen (years) }\end{array}$ & $\begin{array}{c}\text { Tam + Non-optimal } \\
\text { Mam Screen (years) }\end{array}$ & $\begin{array}{c}\text { NoTam + Non-optimal } \\
\text { Mam Screen (years) }\end{array}$ \\
\hline H-Tam & $\$ 6,892.34$ & $\$ 6,199.62$ & N/A \\
H-noTam & $\$ 581.61$ & $\$ 271.61$ & $\$ 865.05$ \\
IBC & $\$ 5,014.86$ & $\$ 4,940.39$ & $\$ 5,383.50$ \\
NIBC & $\$ 1,634.40$ & $\$ 1,634.40$ & $\$ 1,816.14$ \\
EC & $\$ 1,180.19$ & $\$ 1,169.37$ & $\$ 1,088.49$ \\
PE & $\$ 54.36$ & $\$ 54.07$ & $\$ 48.60$ \\
DVT & $\$ 96.59$ & $\$ 95.46$ & $\$ 87.23$ \\
Cat-S & $\$ 236.34$ & $\$ 232.87$ & $\$ 202.73$ \\
Death & $\$ 2,852.96$ & $\$ 2,893.00$ & $\$ 3,082.21$ \\
Total & $\$ 18,543.65$ & $\$ 17,490.79$ & $\$ 12,573.96$ \\
\hline
\end{tabular}

* $3 \%$ discount rate

H-Tam: Healthy with Tamoxifen, H-noTam: Healthy with no Tamoxifen, IBC: Invasive breast cancer, NIBC: Non-invasive breast cancer, EC: Endometrial cancer, PE: Pulmonary embolism, DVT: Deep vein thrombosis, Cat-S: Cataract surgery 
Figure 4.2 is a graphical illustration of simulated costs distribution among three broad combined health states: Healthy (H-Tam and H-noTam), Breast Cancer $(\mathrm{IBC}+\mathrm{NIBC}+$ Death) state, and Non-breast cancer states $(\mathrm{EC}+\mathrm{PE}+\mathrm{DVT}+\mathrm{Cat}-\mathrm{S})$.

Figure 4.2: Simulated Cohort Cost Distribution - Base-case

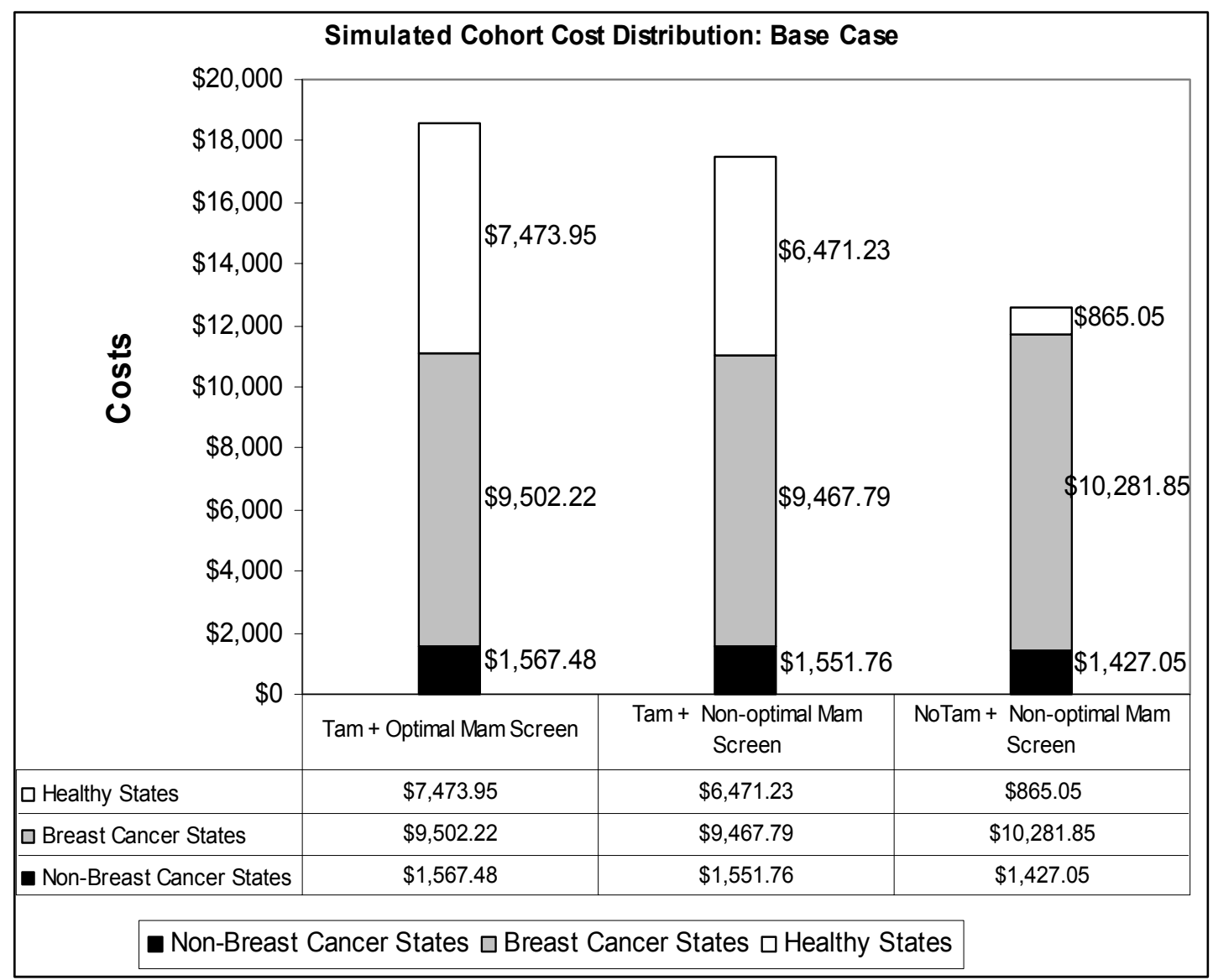

As is evident from the prior discussion, individuals in 'tamoxifen' cohorts experienced a higher average survival than their counterparts in the 'non-tamoxifen' cohort (refer to Table 4.9). However, this improvement in expected survival came at a cost (refer to Table 4.10). Incremental cost-effectiveness analysis was performed in order to determine whether the additional costs that need to be expended for chemopreventive tamoxifen to achieve additional benefits are justifiable. This involved determining incremental cost-effectiveness ratios (ICER). 
An incremental benefit of 0.122 years in the 'Tam + Optimal Mam Screen' cohort compared to 'No-Tam + Non-optimal Mam Screen' cohort at an incremental cost of $\$ 5,969.70$ resulted in an ICER of $\$ 48,931.80$ per life year gained. This estimate is much below the incremental cost-effectiveness ratio benchmark range of $\$ 50,000$ to $\$ 100,000$ per life year gained indicating that chemopreventive tamoxifen along with routine mammography screening is cost effective in breast cancer risk reduction in high risk women (Mark et al., 1995). An incremental benefit of 0.076 years in the 'Tam + Nonoptimal Mam Screen' cohort compared to 'No-Tam + Non-optimal Mam Screen' cohort at an incremental cost of $\$ 4,916.84$ resulted in an ICER of $\$ 64,695.20$ per life year gained. This ICER lies between the initially referred cost effective benchmark range of $\$ 50,000$ to $\$ 100,000$ per life year gained indicating that chemopreventive tamoxifen in presence of sub-optimal mammography screening may be cost effective in breast cancer risk reduction in high risk women (Mark et al., 1995). These results are summarized in Table 4.11. 
Table 4.11: Incremental Cost-effectiveness Ratios for the Cohorts ${ }^{1}$

\begin{tabular}{|c|c|c|c|c|c|}
\hline Cohort & $\begin{array}{l}\text { Life-expectancy } \\
\text { (years) }\end{array}$ & $\begin{array}{c}\text { Incremental } \\
\text { Effectiveness } \\
\text { (years) }\end{array}$ & $\begin{array}{c}\text { Cost } \\
(\$)\end{array}$ & $\begin{array}{c}\text { Incremental } \\
\text { Cost }^{2}\end{array}$ & $\mathrm{ICER}^{2}$ \\
\hline No-Tam + Non-optimal Mam Screen & 21.463 & & $\$ 12,573.95$ & & \\
\hline Tam + Optimal Mam Screen & 21.585 & 0.122 & $\$ 18,543.65$ & $\$ 5,969.70$ & $\$ 48,931.80$ \\
\hline Tam + Non-optimal Mam Screen & 21.539 & 0.076 & $\$ 17,490.79$ & $\$ 4,916.84$ & $\$ 64,695.20$ \\
\hline
\end{tabular}




\section{$\underline{\text { Univariate Sensitivity Analysis Results }}$}

The input estimates, both costs and effectiveness, that were used to populate the Markov model were derived and integrated from various sources. Hence, similar to any other economic model, the present model contains some level of uncertainty. A standard method for dealing with uncertainty of input parameters is sensitivity analysis (Briggs A et al., 1994). For the sensitivity analysis, the input parameters are varied over a certain range and the cost-effectiveness ratios are recalculated. A comparison between the original cost-effectiveness ratio and the sensitivity analysis cost-effectiveness ratios for an input parameter provides indication of how sensitive the overall model results is to changes in that particular parameter. If the results are found to be stable over a reasonable variation in input parameter, the model's conclusions are considered to be robust (Gold M et al., 1996).

Traditional approaches to sensitivity analysis involve univariate analysis in which one input parameter is varied at a time. Sensitivity analysis was first performed on clinical parameters and then on cost parameters. Clinical parameters included in the analysis were: compliance with screening mammography, compliance with chemopreventive tamoxifen, effectiveness of tamoxifen in reducing risk of IBC and NIBC, tamoxifen related adverse event risk, IBC and EC mortality rates.

Results of the 'clinical' parameter sensitivity analysis are reported in Table 4.12. The first row in Table 4.12 are the base-case values and serve as a benchmark against which all the subsequent derived values in the table can be compared. The first column represents the 'clinical' parameters and the pre-specified range across which their values were varied for sensitivity analysis. 
Table 4.12: One-way Sensitivity Analysis at $3 \%$ Discount Rate: Efficacy Parameters

\begin{tabular}{|c|c|c|c|c|c|c|}
\hline Parameter/range & $\begin{array}{c}\text { Cohort } 1^{\S} \\
\text { (Life-years) }\end{array}$ & $\begin{array}{c}\text { Cohort } 2^{\S} \\
\text { (Life-years) }\end{array}$ & 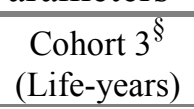 & $\begin{array}{c}\text { Cohort } 1^{\S} \\
\text { (Cost) }\end{array}$ & $\begin{array}{c}\text { Cohort } 2^{\S} \\
(\text { Cost })\end{array}$ & $\begin{array}{c}\text { Cohort } 3^{\S} \\
(\text { Cost })\end{array}$ \\
\hline Base Case & 21.586 & 21.539 & 21.464 & $\$ 18,543.64$ & $\$ 17,491.26$ & $\$ 12,573.96$ \\
\hline \multicolumn{7}{|l|}{ Mammography Compliance } \\
\hline Mammography Compliance ( $20 \%$ down) & 21.586 & 21.531 & 21.455 & $\$ 18,543.64$ & $\$ 17,299.94$ & $\$ 12,385.23$ \\
\hline Mammography Compliance (20\% up) & 21.586 & 21.547 & 21.473 & $\$ 18,543.64$ & $\$ 17,674.74$ & $\$ 12,754.97$ \\
\hline \multicolumn{7}{|l|}{ Tamoxifen Compliance } \\
\hline Tamoxifen non-Compliance (20\% up) & 21.584 & 21.537 & 21.464 & $\$ 18,560.01$ & $\$ 17,507.96$ & $\$ 12,573.96$ \\
\hline Tamoxifen non-Compliance ( $20 \%$ down) & 21.587 & 21.541 & 21.464 & $\$ 18,526.88$ & $\$ 17,474.10$ & $\$ 12,573.96$ \\
\hline \multicolumn{7}{|l|}{ IBC \& NIBC Risk (Tam effectiveness) } \\
\hline $20 \%$ up (worst case) & 21.565 & 21.518 & 21.464 & $\$ 18,728.60$ & $\$ 17,679.00$ & $\$ 12,573.96$ \\
\hline 20\% down (best case) & 21.606 & 21.56 & 21.464 & $\$ 18,357.94$ & $\$ 17,302.10$ & $\$ 12,573.96$ \\
\hline \multicolumn{7}{|l|}{ Adverse Events (EC, PE, DVT,Cat) } \\
\hline $20 \%$ up (worst case) & 21.578 & 21.531 & 21.464 & $\$ 18,633.32$ & $\$ 17,567.43$ & $\$ 12,573.96$ \\
\hline $20 \%$ down (best case) & 21.593 & 21.547 & 21.464 & $\$ 18,453.46$ & $\$ 17,414.93$ & $\$ 12,573.96$ \\
\hline \multicolumn{7}{|l|}{ Mortalities } \\
\hline \multicolumn{7}{|l|}{ IBC } \\
\hline $20 \%$ up & 21.475 & 21.426 & 21.338 & $\$ 18,462.74$ & $\$ 17,408.45$ & $\$ 12,482.08$ \\
\hline $20 \%$ down & 21.715 & 21.673 & 21.612 & $\$ 18,638.74$ & $\$ 17,589.21$ & $\$ 12,682.62$ \\
\hline \multicolumn{7}{|l|}{$\mathbf{E C}$} \\
\hline $20 \%$ up & 21.568 & 21.522 & 21.448 & $\$ 18,445.22$ & $\$ 17,393.67$ & $\$ 12,483.27$ \\
\hline $20 \%$ down & 21.606 & 21.559 & 21.483 & $\$ 18,660.08$ & $\$ 17,606.68$ & $\$ 12,681.25$ \\
\hline
\end{tabular}

$\S$ Cohort 1: Tam + Optimal Mam Screen, Cohort 2: Tam + Non-optimal Mam Screen, Cohort 3: No-Tam + Non-optimal Mam Screen

H-Tam: Healthy with Tamoxifen, H-noTam: Healthy with no Tamoxifen, IBC: Invasive breast cancer, NIBC: Non-invasive breast cancer, EC: Endometrial cancer, PE: Pulmonary embolism, DVT: Deep vein thrombosis, Cat-S: Cataract surgery 
The higher the deviation from the base-case value due to change in the "clinical parameter', the higher is the influence of that parameter on end-points, in this case 'cohort lifeyears' and 'cohort costs'. This ability of 'clinical' parameters to influence 'cohort life years' and 'cohort costs' gets reflected in the final incremental cost-effectiveness ratios which are summarized in Table 4.13. As is evident from Table 4.12, changes in 'clinical' input parameters not only affect the clinical end-point such as 'cohort life-years' but also the 'cohort costs.' This is because the final cost of the cohort not only depends upon cost of treating an individual clinical state but also on amount of time that the cohort spent in that state.

Figures 4.3, 4.4, and 4.5 provide graphical summary of results presented in Table 4.13. These figures illustrate the relationship between change in individual input parameter and their influence on ICER. In all the figures, the $\mathrm{X}$-axis represents the base case estimate and the bars above and below the $\mathrm{X}$-axis represent variation in the base case estimate resulting from variation in the input parameters. As is evident from Figure 4.3, the two input parameters that have the largest impact on ICER between the 'Tam + Optimal Mam Screen' and 'NoTam + Non-optimal Mam Screen' are chemopreventive tamoxifen's effectiveness in reducing risk of IBC and NIBC, and assumption regarding mortality from IBC. Thus, an assumption of an increase in chemopreventive tamoxifen's effectiveness substantially decreases the ICER while an assumption of decreased effectiveness increases the ICER. Figure 4.4 indicates that the two input parameters which have the largest impact on ICER between the 'Tam + Non-optimal Mam Screen' and 'NoTam + Non-optimal Mam Screen' are once again chemopreventive tamoxifen's effectiveness in reducing risk of IBC and NIBC and assumption regarding mortality from IBC. Figure 4.5 indicates that the assumption regarding IBC mortality has the highest impact on the ICER between 'Tam + Optimal Mam Screen' and 'Tam + Non-optimal Mam Screen' 
Table 4.13: One-way Sensitivity Analysis at $3 \%$ Discount Rate: Efficacy Parameters and Incremental Analysis*

\begin{tabular}{|c|c|c|c|}
\hline Parameter/range & $\begin{array}{c}\text { ICER } \\
\text { Cohort } 1 \text { vs. } 3\end{array}$ & $\begin{array}{c}\text { ICER } \\
\text { Cohort } 2 \text { vs. } 3\end{array}$ & $\begin{array}{c}\text { ICER } \\
\text { Cohort } 1 \text { vs. } 2\end{array}$ \\
\hline Base Case & $\$ 48,931.80$ & $\$ 65,564.03$ & $\$ 22,391.02$ \\
\hline \multicolumn{4}{|l|}{ Mammography Compliance } \\
\hline Mammography Compliance ( $20 \%$ up) & $\$ 47,010.76$ & $\$ 64,667.20$ & $\$ 22,612.78$ \\
\hline Mammography Compliance ( $20 \%$ down) & $\$ 51,227.17$ & $\$ 66,483.39$ & $\$ 22,279.46$ \\
\hline \multicolumn{4}{|l|}{ Tamoxifen Compliance } \\
\hline Tamoxifen non-Compliance ( $20 \%$ up) & $\$ 49,883.75$ & $\$ 67,589.08$ & $\$ 22,383.98$ \\
\hline Tamoxifen non-Compliance ( $20 \%$ down) & $\$ 48,397.72$ & $\$ 63,638.18$ & $\$ 22,886.52$ \\
\hline \multicolumn{4}{|l|}{ IBC \& NIBC Risk (Tam effectiveness) } \\
\hline $20 \%$ up (worst case) & $\$ 60,937.07$ & $\$ 94,537.81$ & $\$ 22,331.96$ \\
\hline $20 \%$ down (best case) & $\$ 40,732.30$ & $\$ 49,251.51$ & $\$ 22,953.07$ \\
\hline \multicolumn{4}{|l|}{ Adverse Events (EC, PE, DVT, CAT) } \\
\hline $20 \%$ up (worst case) & $\$ 53,152.28$ & $\$ 74,529.40$ & $\$ 22,678.51$ \\
\hline 20\% down (best case) & $\$ 45,577.52$ & $\$ 58,324.95$ & $\$ 22,576.72$ \\
\hline \multicolumn{4}{|l|}{ Mortalities } \\
\hline \multicolumn{4}{|l|}{ IBC } \\
\hline $20 \%$ up & $\$ 43,654.46$ & $\$ 55,981.47$ & $\$ 21,516.16$ \\
\hline $20 \%$ down & $\$ 57,826.43$ & $\$ 80,435.90$ & $\$ 24,988.86$ \\
\hline \multicolumn{4}{|l|}{ EC } \\
\hline $20 \%$ up & $\$ 49,682.90$ & $\$ 66,356.77$ & $\$ 22,859.72$ \\
\hline $20 \%$ down & $\$ 48,608.38$ & $\$ 64,808.30$ & $\$ 22,412.77$ \\
\hline
\end{tabular}

* all interventions compared to the reference case: No-Tam + Non-optimal Mam Screen

$\S$ Cohort 1: Tam + Optimal Mam Screen, Cohort 2: Tam + Non-optimal Mam Screen, Cohort 3: No-Tam + Non-optimal Mam Screen 
Figure 4.3: Sensitivity Analysis: Tam + Optimal Mam Screen vs. NoTam+Non-optimal Mam Screen

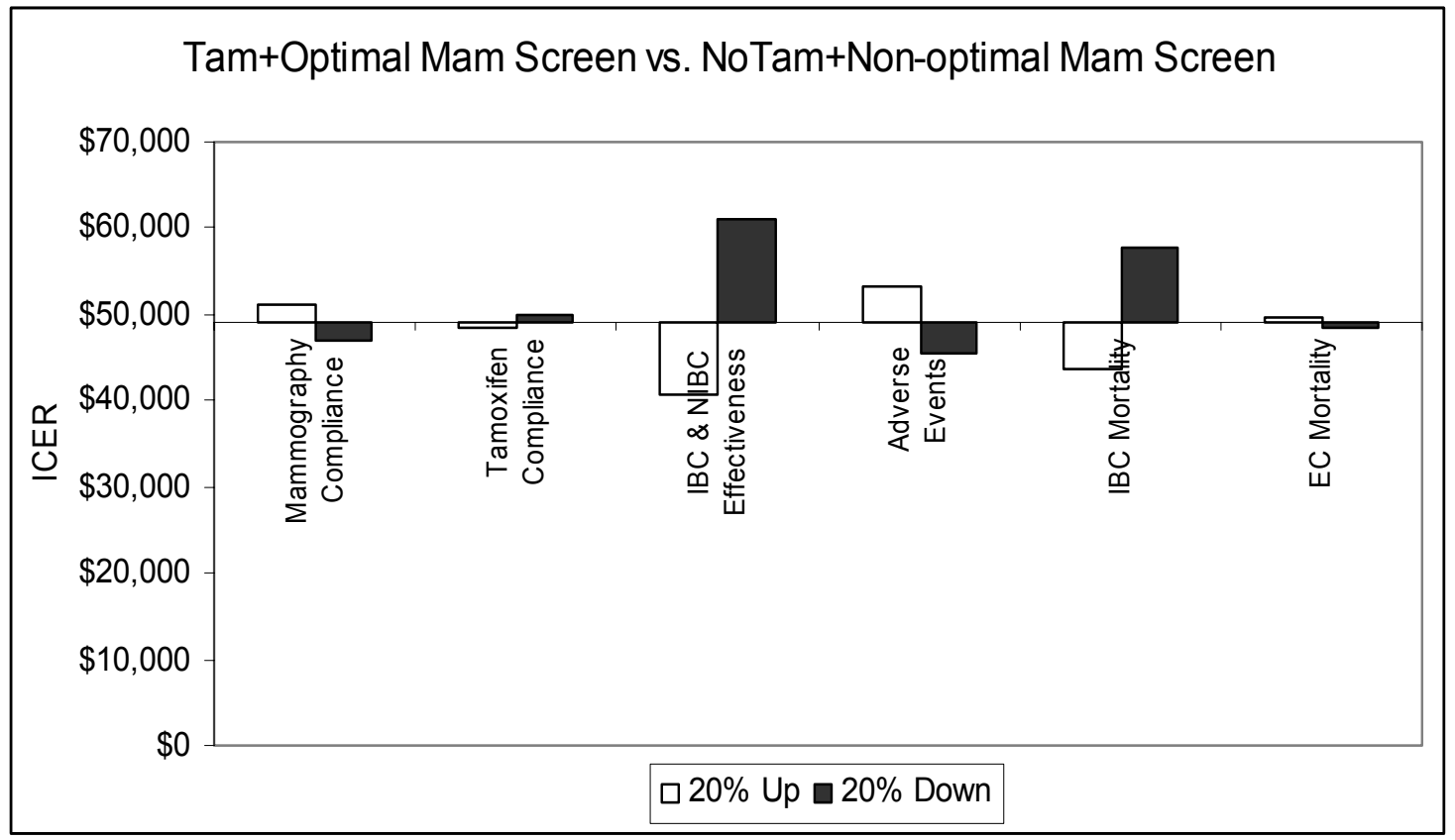

Figure 4.4: Sensitivity Analysis: Tam + Non-optimal Mam Screen vs. NoTam + Nonoptimal Mam Screen

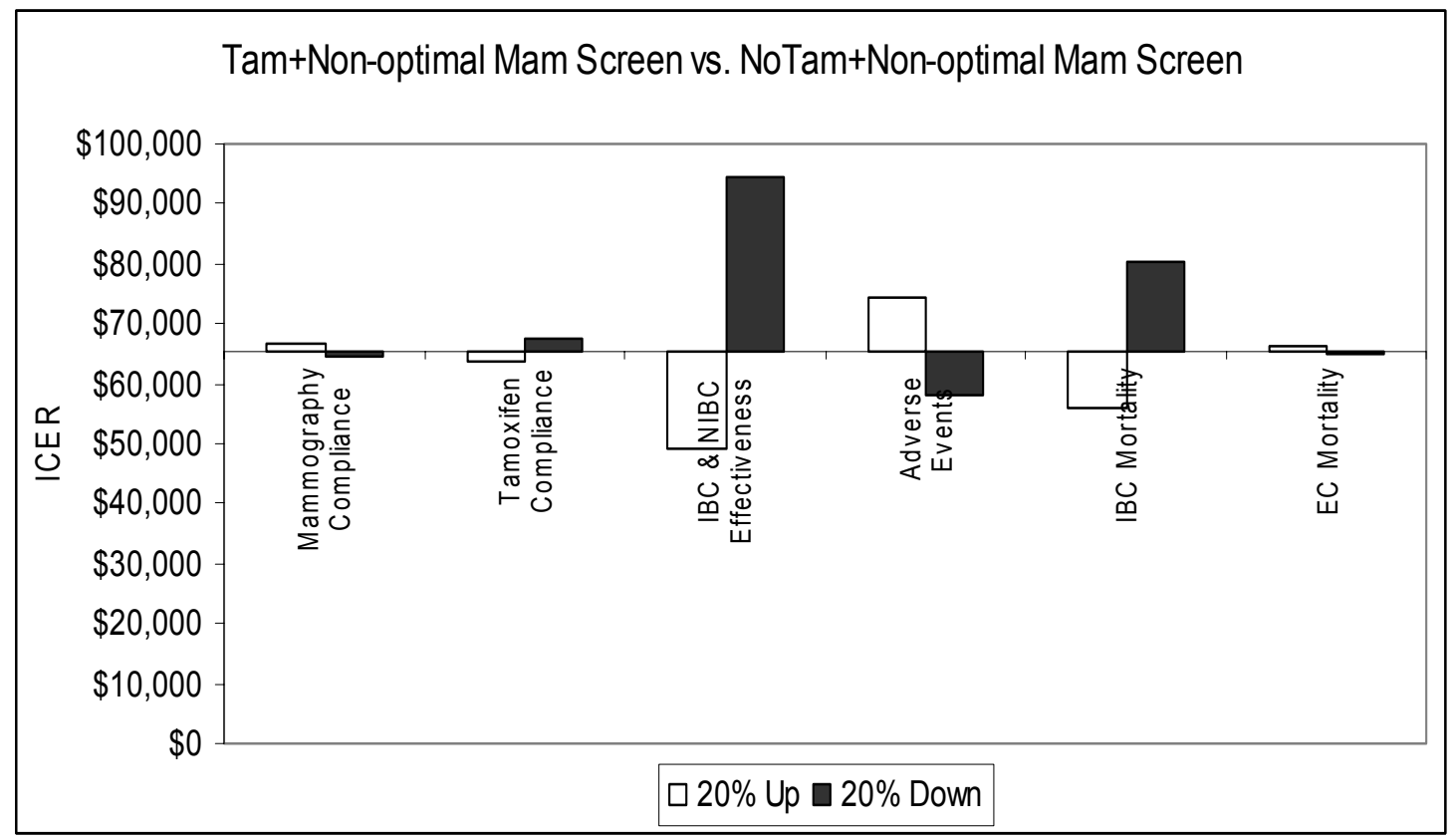


Figure 4.5: Sensitivity Analysis: Tam + Optimal Mam Screen vs. Tam + Non-optimal Mam Screen

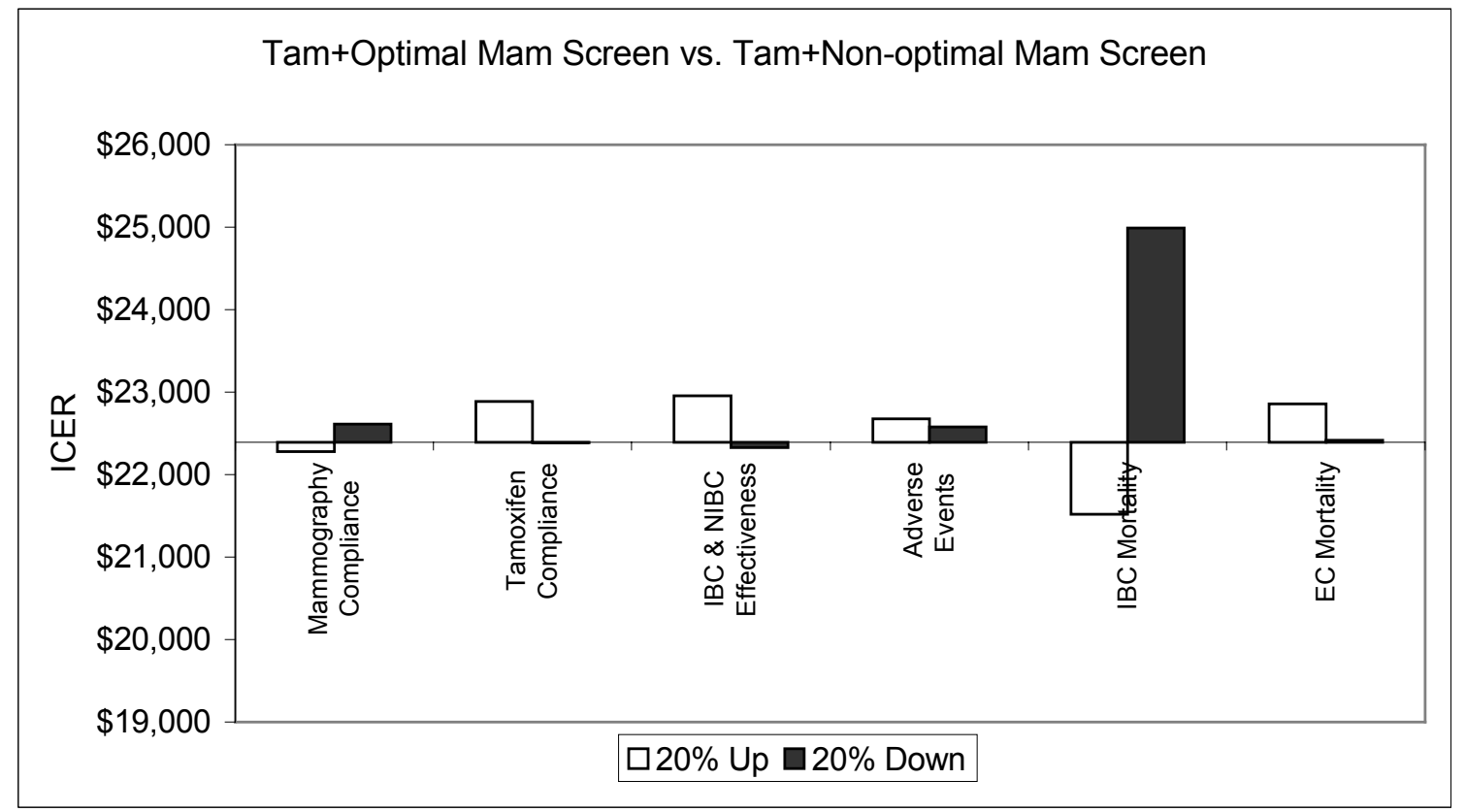


Results of the 'cost' parameter sensitivity analysis are reported in Tables 4.14, 4.15, 4.16, 4.17, 4.18, and 4.19. The first rows in each of these tables are the base-case values and serve as a benchmark against which all the subsequent derived values in the table can be compared to. The first column represents the 'cost' parameters and the pre-specified range across which their values were varied for sensitivity analysis.

The higher the deviation from the base-case value due to change in the 'cost parameter', the higher is the influence of that parameter on end-points, in this case 'cohort costs'. Unlike, clinical parameters, which influence both 'cohort life years' and cohort costs,' 'cost parameters' only influence 'cohort costs' as is evident from following tables. 
Table 4.14: One-way Sensitivity Analysis at $3 \%$ Discount Rate: Cost Parameters for IBC and NIBC

\begin{tabular}{|c|c|c|c|c|c|c|}
\hline Parameter/range & $\begin{array}{c}\text { Cohort } 1^{\S} \\
\text { (Life-years) }\end{array}$ & $\begin{array}{c}\text { Cohort } 2^{\S} \\
\text { (Life-years) }\end{array}$ & $\begin{array}{c}\text { Cohort } 3^{\S} \\
\text { (Life-years) }\end{array}$ & $\begin{array}{c}\text { Cohort } 1^{\S} \\
(\text { Cost })\end{array}$ & $\begin{array}{c}\text { Cohort } 2^{\S} \\
(\text { Cost })\end{array}$ & $\begin{array}{c}\text { Cohort } 3^{\S} \\
\text { (Cost) }\end{array}$ \\
\hline Base case & 21.586 & 21.539 & 21.464 & $\$ 18,543.65$ & $\$ 17,491.26$ & $\$ 12,573.96$ \\
\hline \multicolumn{7}{|l|}{ IBC } \\
\hline \multicolumn{7}{|c|}{ Initial Care Cost } \\
\hline $20 \%$ higher & 21.586 & 21.539 & 21.464 & $\$ 18,963.67$ & $\$ 17,911.27$ & $\$ 13,027.15$ \\
\hline $20 \%$ Lower & 21.586 & 21.539 & 21.464 & $\$ 18,123.63$ & $\$ 17,071.24$ & $\$ 12,120.78$ \\
\hline \multicolumn{7}{|c|}{ Maintenance Cost } \\
\hline $20 \%$ higher & 21.586 & 21.539 & 21.464 & $\$ 19,179.55$ & $\$ 18,113.16$ & $\$ 13,254.93$ \\
\hline $20 \%$ Lower & 21.586 & 21.539 & 21.464 & $\$ 17,906.70$ & $\$ 16,868.32$ & $\$ 11,891.87$ \\
\hline \multicolumn{7}{|c|}{ Terminal Care Cost } \\
\hline $20 \%$ higher & 21.586 & 21.539 & 21.464 & $\$ 18,975.87$ & $\$ 17,930.68$ & $\$ 13,042.97$ \\
\hline $20 \%$ Lower & 21.586 & 21.539 & 21.464 & $\$ 18,111.43$ & $\$ 17,051.83$ & $\$ 12,104.96$ \\
\hline \multicolumn{7}{|l|}{ NIBC } \\
\hline \multicolumn{7}{|c|}{ Initial Care Cost } \\
\hline $20 \%$ higher & 21.586 & 21.539 & 21.464 & $\$ 18,663.55$ & $\$ 17,611.15$ & $\$ 12,704.93$ \\
\hline $20 \%$ Lower & 21.586 & 21.539 & 21.464 & $\$ 18,423.75$ & $\$ 17,371.35$ & $\$ 12,442.99$ \\
\hline \multicolumn{7}{|c|}{ Maintenance Cost } \\
\hline $20 \%$ higher & 21.586 & 21.539 & 21.464 & $\$ 18,762.22$ & $\$ 17,709.82$ & $\$ 12,818.42$ \\
\hline $20 \%$ Lower & 21.586 & 21.539 & 21.464 & $\$ 18,325.08$ & $\$ 17,272.69$ & $\$ 12,329.51$ \\
\hline \multicolumn{7}{|c|}{ Terminal Care Cost } \\
\hline $20 \%$ higher & 21.586 & 21.539 & 21.464 & $\$ 18,616.86$ & $\$ 17,564.47$ & $\$ 12,651.07$ \\
\hline $20 \%$ Lower & 21.586 & 21.539 & 21.464 & $\$ 18,470.44$ & $\$ 17,418.04$ & $\$ 12,496.86$ \\
\hline
\end{tabular}

§ Cohort 1: Tam + Optimal Mam Screen, Cohort 2: Tam + Non-optimal Mam Screen, Cohort 3: No-Tam + Non-optimal Mam Screen 
Table 4.15: One-way Sensitivity Analysis at $3 \%$ Discount Rate: Cost Parameters and Incremental Analysis for IBC and NIBC*

\begin{tabular}{|c|c|c|c|}
\hline Parameter/range & $\begin{array}{c}\text { ICER } \\
\text { Cohort } 1 \text { vs. } 3\end{array}$ & $\begin{array}{c}\text { ICER } \\
\text { Cohort } 2 \text { vs. } 3\end{array}$ & $\begin{array}{c}\text { ICER } \\
\text { Cohort } 1 \text { vs. } 2\end{array}$ \\
\hline Base case & $\$ 48,931.86$ & $\$ 65,563.88$ & $\$ 22,391.40$ \\
\hline \multicolumn{4}{|l|}{ IBC } \\
\hline \multicolumn{4}{|c|}{ Initial Care Cost } \\
\hline $20 \%$ higher & $\$ 48,659.97$ & $\$ 65,121.60$ & $\$ 22,391.40$ \\
\hline $20 \%$ Lower & $\$ 49,203.75$ & $\$ 66,006.15$ & $\$ 22,391.40$ \\
\hline \multicolumn{4}{|c|}{ Maintenance Cost } \\
\hline $20 \%$ higher & $\$ 48,562.40$ & $\$ 64,776.41$ & $\$ 22,688.98$ \\
\hline $20 \%$ Lower & $\$ 49,301.92$ & $\$ 66,352.63$ & $\$ 22,093.34$ \\
\hline \multicolumn{4}{|c|}{ Terminal Care Cost } \\
\hline $20 \%$ higher & $\$ 48,630.34$ & $\$ 65,169.52$ & $\$ 22,238.02$ \\
\hline $20 \%$ Lower & $\$ 49,233.38$ & $\$ 65,958.23$ & $\$ 22,544.79$ \\
\hline \multicolumn{4}{|l|}{ NIBC } \\
\hline \multicolumn{4}{|c|}{ Initial Care Cost } \\
\hline $20 \%$ higher & $\$ 48,841.10$ & $\$ 65,416.24$ & $\$ 22,391.40$ \\
\hline $20 \%$ Lower & $\$ 49,022.62$ & $\$ 65,711.43$ & $\$ 22,391.53$ \\
\hline \multicolumn{4}{|c|}{ Maintenance Cost } \\
\hline $20 \%$ higher & $\$ 48,719.63$ & $\$ 65,218.64$ & $\$ 22,391.43$ \\
\hline $20 \%$ Lower & $\$ 49,144.08$ & $\$ 65,909.10$ & $\$ 22,391.40$ \\
\hline \multicolumn{4}{|c|}{ Terminal Care Cost } \\
\hline $20 \%$ higher & $\$ 48,899.98$ & $\$ 65,512.01$ & $\$ 22,391.40$ \\
\hline $20 \%$ Lower & $\$ 48,963.75$ & $\$ 65,615.75$ & $\$ 22,391.40$ \\
\hline
\end{tabular}

*all interventions compared to the reference case: No-Tam + Non-optimal Mam Screen

Cohort 1: Tam + Optimal Mam Screen, Cohort 2: Tam + Non-optimal Mam Screen, Cohort 3: No-Tam + Non-optimal Mam Screen 
Table 4.16: One-way Sensitivity Analysis at $3 \%$ Discount Rate: Cost Parameters for Non-breast Cancer States

\begin{tabular}{|c|c|c|c|c|c|c|}
\hline Parameter/range & $\begin{array}{c}\text { Cohort } 1^{\S} \\
\text { (Life-years) }\end{array}$ & $\begin{array}{c}\text { Cohort } 2^{\S} \\
\text { (Life-years) }\end{array}$ & $\begin{array}{c}\text { Cohort } 3^{\S} \\
\text { (Life-years) }\end{array}$ & $\begin{array}{c}\text { Cohort } 1^{\S} \\
(\text { Cost })\end{array}$ & $\begin{array}{c}\text { Cohort } 2^{\S} \\
(\text { Cost })\end{array}$ & $\begin{array}{c}\text { Cohort } 3^{\S} \\
\text { (Cost) }\end{array}$ \\
\hline Base case & 21.586 & 21.539 & 21.464 & $\$ 18,543.65$ & $\$ 17,491.26$ & $\$ 12,573.96$ \\
\hline \multicolumn{7}{|l|}{ EC Cost } \\
\hline $20 \%$ higher & 21.586 & 21.539 & 21.464 & $\$ 18,775.63$ & $\$ 17,723.23$ & $\$ 12,789.90$ \\
\hline 20\% Lower & 21.586 & 21.539 & 21.464 & $\$ 18,311.68$ & $\$ 17,259.28$ & $\$ 12,358.03$ \\
\hline \multicolumn{7}{|l|}{ PE Cost } \\
\hline $20 \%$ higher & 21.586 & 21.539 & 21.464 & $\$ 18,554.41$ & $\$ 17,502.02$ & $\$ 12,583.64$ \\
\hline $20 \%$ Lower & 21.586 & 21.539 & 21.464 & $\$ 18,532.89$ & $\$ 17,480.49$ & $\$ 12,564.29$ \\
\hline \multicolumn{7}{|l|}{ DVT Cost } \\
\hline $20 \%$ higher & 21.586 & 21.539 & 21.464 & $\$ 18,562.55$ & $\$ 17,510.15$ & $\$ 12,591.23$ \\
\hline $20 \%$ Lower & 21.586 & 21.539 & 21.464 & $\$ 18,524.76$ & $\$ 17,472.36$ & $\$ 12,556.70$ \\
\hline \multicolumn{7}{|l|}{ Cat-S Cost } \\
\hline $20 \%$ higher & 21.586 & 21.539 & 21.464 & $\$ 18,589.62$ & $\$ 17,537.22$ & $\$ 12,613.98$ \\
\hline 20\% Lower & 21.586 & 21.539 & 21.464 & $\$ 18,497.69$ & $\$ 17,445.29$ & $\$ 12,533.95$ \\
\hline
\end{tabular}

§ Cohort 1: Tam + Optimal Mam Screen, Cohort 2: Tam + Non-optimal Mam Screen, Cohort 3: No-Tam + Non-optimal Mam Screen 
Table 4.17: One-way Sensitivity Analysis at $3 \%$ Discount Rate: Cost Parameters and Incremental Analysis for Non-Breast Cancer States*

\begin{tabular}{|c|c|c|c|}
\hline Parameter/range & $\begin{array}{c}\text { ICER } \\
\text { Cohort } 1 \text { vs. } 3\end{array}$ & $\begin{array}{c}\text { ICER } \\
\text { Cohort } 2 \text { vs. } 3\end{array}$ & $\begin{array}{c}\text { ICER } \\
\text { Cohort } 1 \text { vs. } 2\end{array}$ \\
\hline Base case & $\$ 48,931.86$ & $\$ 65,563.88$ & $\$ 22,391.40$ \\
\hline \multicolumn{4}{|l|}{ EC Cost } \\
\hline $20 \%$ higher & $\$ 49,063.36$ & $\$ 65,777.78$ & $\$ 22,391.40$ \\
\hline $20 \%$ Lower & $\$ 48,800.35$ & $\$ 65,349.96$ & $\$ 22,391.40$ \\
\hline \multicolumn{4}{|l|}{ PE Cost } \\
\hline $20 \%$ higher & $\$ 48,940.75$ & $\$ 65,578.37$ & $\$ 22,391.36$ \\
\hline 20\% Lower & $\$ 48,922.94$ & $\$ 65,549.33$ & $\$ 22,391.47$ \\
\hline \multicolumn{4}{|l|}{ DVT Cost } \\
\hline $20 \%$ higher & $\$ 48,945.21$ & $\$ 65,585.59$ & $\$ 22,391.41$ \\
\hline $20 \%$ Lower & $\$ 48,918.50$ & $\$ 65,542.15$ & $\$ 22,391.40$ \\
\hline \multicolumn{4}{|l|}{ Cat-S Cost } \\
\hline $20 \%$ higher & $\$ 48,980.62$ & $\$ 65,643.18$ & $\$ 22,391.41$ \\
\hline $20 \%$ Lower & $\$ 48,883.09$ & $\$ 65,484.55$ & $\$ 22,391.40$ \\
\hline
\end{tabular}

* all interventions compared to the reference case: No-Tam + Non-optimal Mam Screen

Cohort 1: No-Tam + Non-optimal Mam Screen, Cohort 2: Tam + Optimal Mam Screen, Cohort 3: Tam + Non-optimal Mam Screen 
Table 4.18: One-way Sensitivity Analysis at $3 \%$ Discount Rate: Cost Parameters for Intervention Strategies

\begin{tabular}{|c|c|c|c|c|c|c|}
\hline Parameter/range & $\begin{array}{c}\text { Cohort } 1^{\S} \\
\text { (Life-years) }\end{array}$ & $\begin{array}{c}\text { Cohort } 2^{\S} \\
\text { (Life-years) }\end{array}$ & $\begin{array}{c}\text { Cohort } 3^{\S} \\
\text { (Life-years) }\end{array}$ & $\begin{array}{c}\text { Cohort } 1^{\S} \\
(\text { Cost })\end{array}$ & $\begin{array}{c}\text { Cohort } 2^{\S} \\
\text { (Cost) }\end{array}$ & $\begin{array}{c}\text { Cohort } 3^{\S} \\
\text { (Cost) }\end{array}$ \\
\hline Base case & 21.586 & 21.539 & 21.464 & $\$ 18,543.65$ & $\$ 17,491.26$ & $\$ 12,573.96$ \\
\hline \multicolumn{7}{|l|}{ Tamoxifen Cost } \\
\hline $20 \%$ higher & 21.586 & 21.539 & 21.464 & $\$ 19,662.18$ & $\$ 18,609.78$ & $\$ 12,573.96$ \\
\hline $20 \%$ Lower & 21.586 & 21.539 & 21.464 & $\$ 17,425.12$ & $\$ 16,372.72$ & $\$ 12,573.96$ \\
\hline \multicolumn{7}{|c|}{ Mammography Screening Cost } \\
\hline $20 \%$ higher & 21.586 & 21.539 & 21.464 & $\$ 18,925.88$ & $\$ 17,669.76$ & $\$ 12,749.53$ \\
\hline 20\% Lower & 21.586 & 21.539 & 21.464 & $\$ 18,161.61$ & $\$ 17,312.84$ & $\$ 12,398.49$ \\
\hline
\end{tabular}


Table 4.19: One-way Sensitivity Analysis at 3 \% Discount Rate: Cost Parameters and Incremental Analysis for Intervention Strategies*

\begin{tabular}{lccc}
\hline Parameter/range & $\begin{array}{c}\text { ICER } \\
\text { Cohort 1 vs. 3 }\end{array}$ & $\begin{array}{c}\text { ICER } \\
\text { Cohort 2 vs. 3 }\end{array}$ & $\begin{array}{c}\text { ICER } \\
\text { Cohort 1 vs. 2 }\end{array}$ \\
\hline Base case & $\$ 48,931.86$ & $\$ 65,563.88$ & $\$ 22,391.40$ \\
Tamoxifen Cost & & & \\
$20 \%$ higher & $\$ 58,100.14$ & $\$ 80,477.61$ & $\$ 22,391.41$ \\
$20 \%$ Lower & $\$ 39,763.58$ & $\$ 50,650.14$ & $\$ 22,391.41$ \\
Mammography Screening Cost & & & \\
$20 \%$ higher & $\$ 50,625.83$ & $\$ 65,603.00$ & $\$ 26,726.09$ \\
$20 \%$ Lower & $\$ 47,238.74$ & $\$ 65,524.75$ & $\$ 18,058.94$ \\
\hline
\end{tabular}

* all interventions compared to the reference case: No-Tam + Non-optimal Mam Screen

Cohort 1: No-Tam + Non-optimal Mam Screen, Cohort 2: Tam + Optimal Mam Screen, Cohort 3: Tam + Non-optimal Mam Screen 
Figures 4.6, 4.7, and 4.8 provide graphical summary of results presented in Tables 4.15, 4.17, and 4.19. In all the figures, the $\mathrm{X}$-axis represents the base case estimate and the bars above and below the $\mathrm{X}$-axis represent variation in the base case estimate resulting from variation in the input parameters.

As is evident from Figure 4.6, the two input parameters that have the largest impact on ICER between the 'Tam + Optimal Mam Screen' and 'NoTam + Non-optimal Mam Screen' are cost of chemopreventive tamoxifen and cost of mammography screening.

Figure 4.7 indicates that the single most influential input parameter that substantially affects the ICER between 'Tam + Non-optimal Mam Screen' and 'NoTam + Non-optimal Mam Screen' is chemopreventive tamoxifen cost.

Figure 4.8 indicates that the single most influential input parameter that substantially affects the ICER between 'Tam + Non-optimal Mam Screen' and 'NoTam + Non-optimal Mam Screen' is cost of mammography screening. 
Figure 4.6: Sensitivity Analysis: Tam + Optimal Mam Screen vs. NoTam + Non-optimal Mam Screen

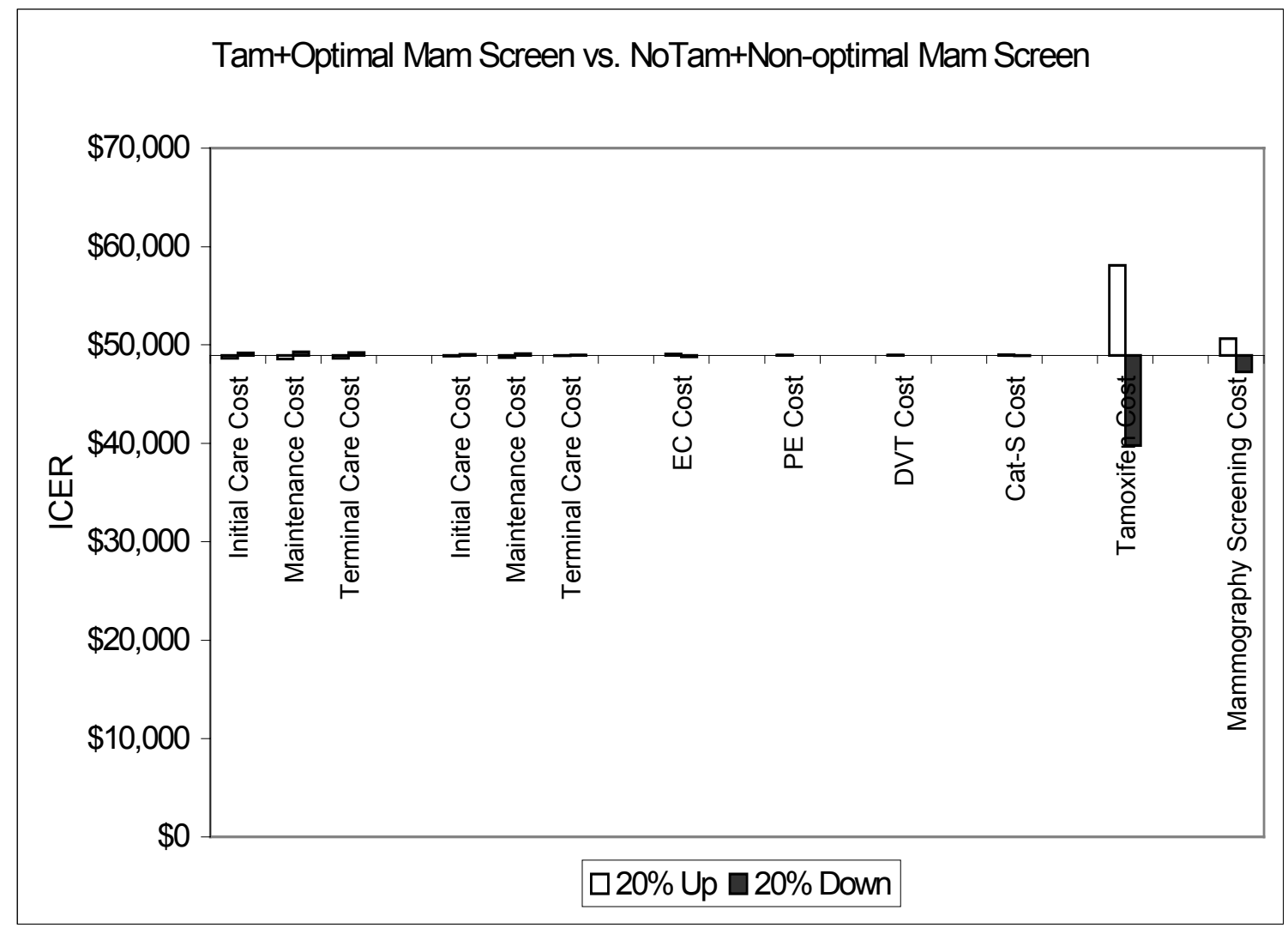


Figure 4.7: Sensitivity Analysis: Tam + Non-optimal Mam Screen vs. NoTam + Nonoptimal Mam Screen

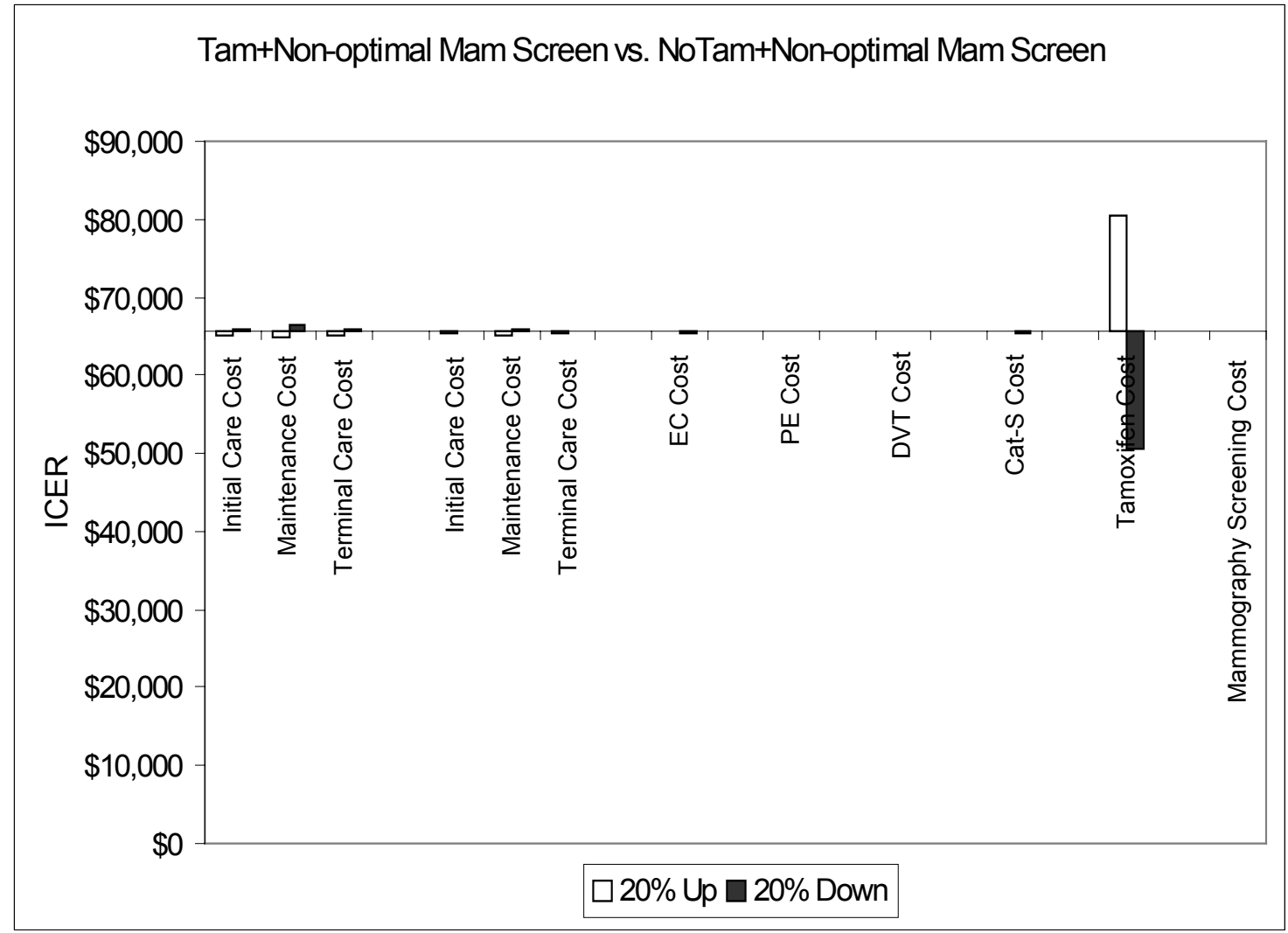


Figure 4.8: Sensitivity Analysis: Tam + Optimal Mam Screen vs. Tam + Non-optimal Mam Screen

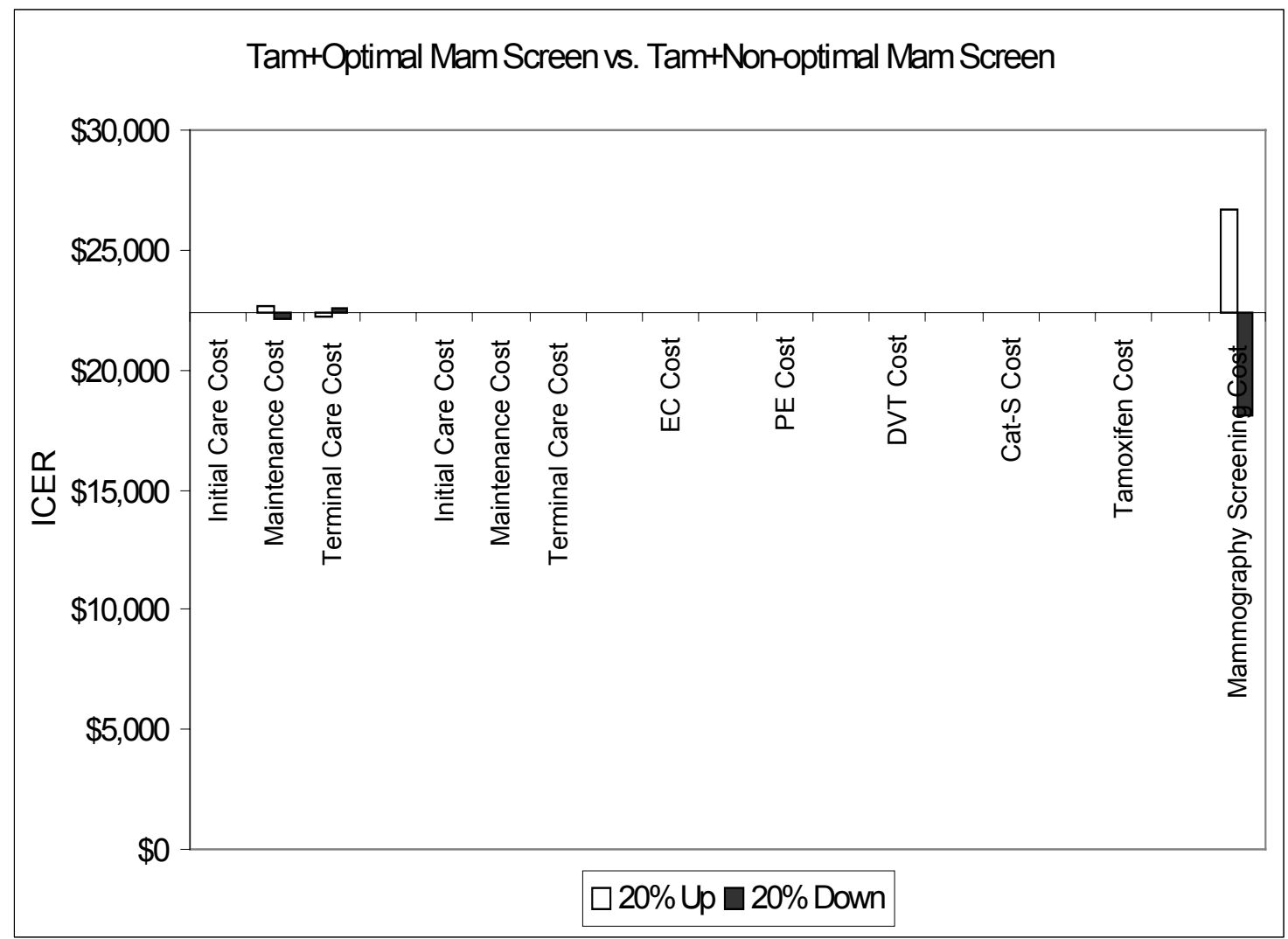


Discounting

Discounting principle is based on the assumption that costs that are incurred and benefits that are realized in the present time are not equivalent to the ones that are incurred or realized in some point in the future (Kielhorn A, Graf van der Schulenburg, 2000). A discount rate of $3 \%$ was used to perform the base case analysis based on recommendations of the "Panel on Cost-effectiveness in Health and Medicine"(Gold M et al., 1996). However, the same panel also recommended using the traditional discount rate of $5 \%$ in the sensitivity analysis as a large number of studies have used this discount rate in their analysis. Hence, results using $5 \%$ discount rate have been presented in Table 4.20 and 4.21. The same table also reports results of bivariate sensitivity analysis, which was performed changing both the discounting rate and the individual clinical parameter at the same time. Thus, the first row in Table 4.20 reports univariate sensitivity analysis results of changing the discount rate alone while all rows thereafter report the bivariate results.

In addition to using, $5 \%$ discount rate, the analysis was also performed at $0 \%$ discount rate (undiscounted costs and benefits), the results of which are reported in Tables 4.22 and 4.23 . The base case results using $0 \%$ discounting rate are reported in the first row followed by bivariate sensitivity analysis results in the rows thereafter. 
Table 4.20: One-way Sensitivity Analysis at $5 \%$ Discount Rate: Efficacy Parameters

\begin{tabular}{|c|c|c|c|c|c|c|}
\hline Parameter/range & $\begin{array}{c}\text { Cohort } 1^{\S} \\
\text { (Life-years) }\end{array}$ & $\begin{array}{c}\text { Cohort } 2^{\S} \\
\text { (Life-years) }\end{array}$ & $\begin{array}{c}\text { Cohort } 3^{\S} \\
\text { (Life-years) }\end{array}$ & $\begin{array}{c}\text { Cohort } 1^{\S} \\
(\text { Cost })\end{array}$ & $\begin{array}{c}\text { Cohort } 2^{\S} \\
(\text { Cost })\end{array}$ & $\begin{array}{c}\text { Cohort } 3^{\S} \\
(\text { Cost })\end{array}$ \\
\hline Base Case & 16.145 & 16.118 & 16.070 & $\$ 14,308.99$ & $\$ 13,530.30$ & $\$ 8,509.82$ \\
\hline \multicolumn{7}{|l|}{ Mammography Compliance } \\
\hline Mammography Compliance ( $20 \%$ down) & 16.145 & 16.114 & 16.075 & $\$ 14,308.99$ & $\$ 13,388.61$ & $\$ 8,643.56$ \\
\hline Mammography Compliance (20\% up) & 16.145 & 16.123 & 16.065 & $\$ 14,308.99$ & $\$ 13,666.15$ & $\$ 8,370.33$ \\
\hline \multicolumn{7}{|l|}{ Tamoxifen Compliance } \\
\hline Tamoxifen non-Compliance (20\% up) & 16.143 & 16.117 & 16.070 & $\$ 14,322.54$ & $\$ 13,544.14$ & $\$ 8,509.82$ \\
\hline Tamoxifen non-Compliance ( $20 \%$ down) & 16.146 & 16.119 & 16.070 & $\$ 14,295.08$ & $\$ 13,516.09$ & $\$ 8,509.82$ \\
\hline \multicolumn{7}{|l|}{ IBC \& NIBC Risk (Tam effectiveness) } \\
\hline $20 \%$ up (worst case) & 16.132 & 16.105 & 16.070 & $\$ 14,466.35$ & $\$ 13,690.63$ & $\$ 8,509.82$ \\
\hline $20 \%$ down (best case) & 16.158 & 16.132 & 16.070 & $\$ 14,151.00$ & $\$ 13,369.34$ & $\$ 8,509.82$ \\
\hline \multicolumn{7}{|l|}{ Adverse Events (EC, PE, DVT,Cat) } \\
\hline $20 \%$ up (worst case) & 16.14 & 16.113 & 16.070 & $\$ 14,383.91$ & $\$ 13,592.41$ & $\$ 8,509.82$ \\
\hline $20 \%$ down (best case) & 16.15 & 16.123 & 16.070 & $\$ 14,233.66$ & $\$ 13,468.06$ & $\$ 8,509.82$ \\
\hline \multicolumn{7}{|l|}{ Mortalities } \\
\hline \multicolumn{7}{|l|}{ IBC } \\
\hline $20 \%$ up & 16.082 & 16.054 & 15.998 & $\$ 14,299.69$ & $\$ 13,520.74$ & $\$ 8,499.04$ \\
\hline $20 \%$ down & 16.216 & 16.192 & 16.154 & $\$ 14,319.70$ & $\$ 13,541.37$ & $\$ 8,522.31$ \\
\hline \multicolumn{7}{|l|}{$\mathrm{EC}$} \\
\hline $20 \%$ up & 16.134 & 16.108 & 16.061 & $\$ 14,250.12$ & $\$ 13,471.94$ & $\$ 8,455.92$ \\
\hline $20 \%$ down & 16.156 & 16.130 & 16.081 & $\$ 14,377.23$ & $\$ 13,597.96$ & $\$ 8,572.32$ \\
\hline
\end{tabular}

$\S$ Cohort 1: Tam + Optimal Mam Screen, Cohort 2: Tam + Non-optimal Mam Screen, Cohort 3: No-Tam + Non-optimal Mam Screen 
Table 4.21: One-way Sensitivity Analysis at $5 \%$ Discount Rate: Efficacy Parameters and Incremental Analysis*

\begin{tabular}{|c|c|c|c|}
\hline Parameter/range & $\begin{array}{c}\text { ICER } \\
\text { Cohort } 2 \text { vs. } 1\end{array}$ & $\begin{array}{c}\text { ICER } \\
\text { Cohort } 3 \text { vs. } 1\end{array}$ & $\begin{array}{c}\text { ICER } \\
\text { Cohort } 2 \text { vs. } 3\end{array}$ \\
\hline Base Case & $\$ 77,322.24$ & $\$ 104,593.33$ & $\$ 28,840.30$ \\
\hline \multicolumn{4}{|l|}{ Mammography Compliance } \\
\hline Mammography Compliance (20\% down) & $\$ 80,934.71$ & $\$ 121,668.00$ & $\$ 29,689.61$ \\
\hline Mammography Compliance (20\% up) & $\$ 74,326.05$ & $\$ 91,464.89$ & $\$ 29,219.73$ \\
\hline \multicolumn{4}{|l|}{ Tamoxifen Compliance } \\
\hline Tamoxifen non-Compliance (20\% up) & $\$ 79,626.32$ & $\$ 107,113.30$ & $\$ 29,938.31$ \\
\hline Tamoxifen non-Compliance ( $20 \%$ down) & $\$ 76,121.82$ & $\$ 102,168.78$ & $\$ 28,851.41$ \\
\hline \multicolumn{4}{|l|}{ IBC \& NIBC Risk (Tam effectiveness) } \\
\hline $20 \%$ up (worst case) & $\$ 96,073.06$ & $\$ 148,023.34$ & $\$ 28,730.11$ \\
\hline $20 \%$ down (best case) & $\$ 64,104.35$ & $\$ 78,379.42$ & $\$ 30,063.81$ \\
\hline \multicolumn{4}{|l|}{ Adverse Events (EC, PE, DVT,Cat) } \\
\hline $20 \%$ up (worst case) & $\$ 83,915.54$ & $\$ 118,199.91$ & $\$ 29,314.52$ \\
\hline $20 \%$ down (best case) & $\$ 71,547.98$ & $\$ 93,551.75$ & $\$ 28,355.37$ \\
\hline \multicolumn{4}{|l|}{ Mortalities } \\
\hline \multicolumn{4}{|l|}{ IBC } \\
\hline $20 \%$ up & $\$ 69,055.36$ & $\$ 89,673.30$ & $\$ 27,819.46$ \\
\hline $20 \%$ down & $\$ 93,506.31$ & $\$ 132,080.71$ & $\$ 32,430.17$ \\
\hline \multicolumn{4}{|l|}{ EC } \\
\hline $20 \%$ up & $\$ 79,372.66$ & $\$ 106,723.79$ & $\$ 29,930.23$ \\
\hline $20 \%$ down & $\$ 77,398.81$ & $\$ 102,564.04$ & $\$ 29,972.04$ \\
\hline
\end{tabular}

* all interventions compared to the reference case: No-Tam + Non-optimal Mam Screen

$\S$ Cohort 1: Tam + Optimal Mam Screen, Cohort 2: Tam + Non-optimal Mam Screen, Cohort 3: No-Tam + Non-optimal Mam Screen 
Table 4.22: One-way Sensitivity Analysis at $0 \%$ Discount Rate: Efficacy Parameters

\begin{tabular}{|c|c|c|c|c|c|c|}
\hline Parameter/range & $\begin{array}{c}\text { Cohort } 1^{\S} \\
\text { (Life-years) }\end{array}$ & $\begin{array}{c}\text { Cohort } 2^{\S} \\
\text { (Life-years) }\end{array}$ & $\begin{array}{c}\text { Cohort } 3^{\S} \\
\text { (Life-years) }\end{array}$ & $\begin{array}{c}\text { Cohort } 1^{\S} \\
(\text { Cost })\end{array}$ & $\begin{array}{c}\text { Cohort } 2^{\S} \\
(\text { Cost })\end{array}$ & $\begin{array}{c}\text { Cohort } 3^{\S} \\
(\text { Cost })\end{array}$ \\
\hline Base Case & 37.252 & 37.135 & 36.974 & $\$ 32,603.11$ & $\$ 30,712.25$ & $\$ 26,074.74$ \\
\hline \multicolumn{7}{|l|}{ Mammography Compliance } \\
\hline Mammography Compliance ( $20 \%$ down) & 37.252 & 37.115 & 36.995 & $\$ 32,603.11$ & $\$ 30,369.34$ & $\$ 26,401.11$ \\
\hline Mammography Compliance (20\% up) & 37.252 & 37.155 & 36.952 & $\$ 32,603.11$ & $\$ 31,041.36$ & $\$ 25,734.72$ \\
\hline \multicolumn{7}{|l|}{ Tamoxifen Compliance } \\
\hline Tamoxifen non-Compliance ( $20 \%$ up) & 37.248 & 37.131 & 36.974 & $\$ 32,627.16$ & $\$ 30,736.66$ & $\$ 26,074.74$ \\
\hline Tamoxifen non-Compliance ( $20 \%$ down) & 37.256 & 37.139 & 36.974 & $\$ 32,578.40$ & $\$ 30,687.17$ & $\$ 26,074.74$ \\
\hline \multicolumn{7}{|l|}{ IBC \& NIBC Risk (Tam effectiveness) } \\
\hline $20 \%$ up (worst case) & 37.209 & 37.09 & 36.974 & $\$ 32,861.70$ & $\$ 30,974.47$ & $\$ 26,074.74$ \\
\hline $20 \%$ down (best case) & 37.295 & 37.181 & 36.974 & $\$ 32,343.46$ & $\$ 30,448.96$ & $\$ 26,074.74$ \\
\hline \multicolumn{7}{|l|}{ Adverse Events (EC, PE, DVT,Cat) } \\
\hline $20 \%$ up (worst case) & 37.235 & 37.119 & 36.974 & $\$ 32,711.96$ & $\$ 30,821.88$ & $\$ 26,074.74$ \\
\hline 20\% down (best case) & 37.269 & 37.152 & 36.974 & $\$ 32,494.16$ & $\$ 30,602.40$ & $\$ 26,074.74$ \\
\hline \multicolumn{7}{|l|}{ Mortalities } \\
\hline \multicolumn{7}{|l|}{ IBC } \\
\hline $20 \%$ up & 36.962 & 36.841 & 36.654 & $\$ 32,138.87$ & $\$ 30,240.35$ & $\$ 25,561.97$ \\
\hline $20 \%$ down & 37.602 & 37.493 & 37.363 & $\$ 33,167.19$ & $\$ 31,289.31$ & $\$ 26,702.01$ \\
\hline \multicolumn{7}{|l|}{ EC } \\
\hline $20 \%$ up & 37.211 & 37.094 & 36.935 & $\$ 32,367.08$ & $\$ 30,478.22$ & $\$ 25,855.41$ \\
\hline $20 \%$ down & 37.302 & 37.185 & 37.020 & $\$ 32,891.84$ & $\$ 30,998.40$ & $\$ 26,342.92$ \\
\hline
\end{tabular}

§ Cohort 1: Tam + Optimal Mam Screen, Cohort 2: Tam + Non-optimal Mam Screen, Cohort 3: No-Tam + Non-optimal Mam Screen 
Table 4.23: One-way Sensitivity Analysis at $0 \%$ Discount Rate: Efficacy Parameters and Incremental Analysis*

\begin{tabular}{|c|c|c|c|}
\hline Parameter/range & $\begin{array}{c}\text { ICER } \\
\text { Cohort } 2 \text { vs. } 1\end{array}$ & $\begin{array}{c}\text { ICER } \\
\text { Cohort } 3 \text { vs. } 1\end{array}$ & $\begin{array}{c}\text { ICER } \\
\text { Cohort } 2 \text { vs. } 3\end{array}$ \\
\hline Base Case & $\$ 23,483.35$ & $\$ 28,804.44$ & $\$ 16,161.17$ \\
\hline \multicolumn{4}{|l|}{ Mammography Compliance } \\
\hline Mammography Compliance ( $20 \%$ down) & $\$ 24,132.30$ & $\$ 33,068.64$ & $\$ 16,304.84$ \\
\hline Mammography Compliance (20\% up) & $\$ 22,894.63$ & $\$ 26,141.10$ & $\$ 16,100.46$ \\
\hline \multicolumn{4}{|l|}{ Tamoxifen Compliance } \\
\hline Tamoxifen non-Compliance (20\% up) & $\$ 23,913.97$ & $\$ 29,693.81$ & $\$ 16,158.12$ \\
\hline Tamoxifen non-Compliance ( $20 \%$ down) & $\$ 23,062.63$ & $\$ 27,954.16$ & $\$ 16,164.32$ \\
\hline \multicolumn{4}{|l|}{ IBC \& NIBC Risk (Tam effectiveness) } \\
\hline $20 \%$ up (worst case) & $\$ 28,880.69$ & $\$ 42,239.09$ & $\$ 15,859.04$ \\
\hline $20 \%$ down (best case) & $\$ 19,528.75$ & $\$ 21,131.53$ & $\$ 16,618.44$ \\
\hline \multicolumn{4}{|l|}{ Adverse Events (EC, PE, DVT,Cat) } \\
\hline $20 \%$ up (worst case) & $\$ 25,429.98$ & $\$ 32,738.90$ & $\$ 16,293.83$ \\
\hline $20 \%$ down (best case) & $\$ 21,760.77$ & $\$ 25,436.31$ & $\$ 16,168.92$ \\
\hline \multicolumn{4}{|l|}{ Mortalities } \\
\hline \multicolumn{4}{|l|}{ IBC } \\
\hline $20 \%$ up & $\$ 21,353.56$ & $\$ 25,018.07$ & $\$ 15,690.23$ \\
\hline $20 \%$ down & $\$ 27,050.95$ & $\$ 35,286.95$ & $\$ 17,228.19$ \\
\hline \multicolumn{4}{|l|}{ EC } \\
\hline $20 \%$ up & $\$ 23,593.00$ & $\$ 29,074.29$ & $\$ 16,144.06$ \\
\hline $20 \%$ down & $\$ 23,223.10$ & $\$ 28,214.99$ & $\$ 16,183.26$ \\
\hline
\end{tabular}

* all interventions compared to the reference case: No-Tam + Non-optimal Mam Screen

$\S$ Cohort 1: Tam + Optimal Mam Screen, Cohort 2: Tam + Non-optimal Mam Screen, Cohort 3: No-Tam + Non-optimal Mam Screen 
Sensitivity analysis suggested that the overall results of the model were not substantially affected over the range of variables confirming that the study results were robust.

Tamoxifen in Hysterectomized Women (sub-group analysis)

One of the major side effects of tamoxifen is an increased risk of endometrial cancer (Fischer et al., 1998). Hence, an additional analysis was performed to determine incremental survival benefits and incremental cost per life years gained in hysterectomized women who were healthy but at a higher risk of developing breast cancer. As there is no risk of developing endometrial cancer in this cohort of women, a 'zero' probability of transiting to disease state 'EC' was assigned to both 'chemopreventive tamoxifen plus mammography screening' cohort and 'mammography screening' cohort. Chemopreventive tamoxifen plus screening mammography with optimal compliance was found to yield an average survival benefit of 0.129 years (about 47 days) at an incremental cost of $\$ 5,897$ resulting in ICER of $\$ 45,712$ per life year gained. Similarly, chemopreventive tamoxifen plus screening mammography at rates observed in real-world setting was found to yield an average survival benefit of 0.083 years (about 30 days) at an incremental cost of $\$ 4,840$ resulting in ICER of $\$ 58,308$ per life year gained. As is evident, the ICERs in hysterectomized women was slightly better than in general women population who were at a high risk of developing breast cancer. These lower ICERs can be explained on basis that the increased risk of endometrial cancer in chemopreventive tamoxifen group, which had a diminishing effect on average survival and positive effect on cost, was eliminated in the hysterectomized women.

\section{Phase II}

Phase II of this study involved a cross sectional mail survey of randomly selected white women 40 years of age and older who were enrollees of West Virginia Medicaid Program (WVMP). A total of 2,000 surveys were mailed initially followed by a second and a third mailing to all non respondents from previous mailings. Out of the 2,000 surveys, a total of 498 surveys were returned due to wrong address resulting in a reachable sample size of 1502 . A total of 606 responses were obtained from three 
mailings yielding a response rate of $40.34 \%$. However, 25 women among the 606 respondents noted they were diagnosed with invasive breast cancer at some point in time and were excluded from further analysis yielding an eligibility rate of $95.87 \%$ (eligibility rate $=581 / 606)$.

A total of 41 women returned the non-respondent survey. The most common reasons for non-response was 'I do not respond to surveys' followed by 'lost the survey,' 'I am not interested in this issue.' A student's t-test analysis that was performed to compare any age differences among the respondents and non-respondents revealed that there was no significant differences between the two groups (respondent's mean age: 55.2 years (SD: 7.62 years) vs. non-respondent's age: 56.9 (SD: 6.05 years), $\mathrm{p}=0.165$ ). Furthermore, there were no differences in terms of 'proportion of women at high risk of developing breast cancer' in the two groups.

\section{Data Analysis}

Data analysis was done using Statistical Package for Social Sciences - Version 10 software (Copyright (C) 2002, SPSS Inc.). Average age of responding women was about 55.2 years (SD: 7.62 years, range: 27 years - 68 years) out of which $26.5 \%$ were 49 years of age or less, $40.0 \%$ were between 50 to 59 years of age, and $33.5 \%$ were 60 years or older. Table 4.24 reports the baseline characteristics of all responding women who met the eligibility criteria of not having invasive breast cancer. 
Table 4.24: Characteristics of All Respondents

\begin{tabular}{|c|c|}
\hline Item/Variable & N (Valid \%) \\
\hline \multicolumn{2}{|l|}{ Breast Cancer Prevention Behavior } \\
\hline Yes & $521(90.5 \%)$ \\
\hline No & $54(9.3 \%)$ \\
\hline Not sure & $01(0.2 \%)$ \\
\hline Total & $576(100.0 \%)$ \\
\hline \multicolumn{2}{|l|}{ How long since the last mammography } \\
\hline Less than a year & $259(50.7 \%)$ \\
\hline$>1$ year but $<2$ years & $132(25.8 \%)$ \\
\hline$>2$ years & $120(23.5 \%)$ \\
\hline Total & $511(100.0 \%)$ \\
\hline \multicolumn{2}{|l|}{ Ever taken tamoxifen } \\
\hline$\overline{\text { Yes }}$ & $02(0.3 \%)$ \\
\hline No & $556(96.7 \%)$ \\
\hline Not sure & $17(3.0 \%)$ \\
\hline Total & $575(100.0 \%)$ \\
\hline \multicolumn{2}{|l|}{ Actual Risk of Breast Cancer ${ }^{1}$} \\
\hline Women not at high risk $(5$-year risk $<1.7 \%)$ & $258(46.7 \%)$ \\
\hline Women at high risk $(5$-year risk $\geq 1.7 \%)$ & $295(53.3 \%)$ \\
\hline Total & $553(100.0 \%)$ \\
\hline \multicolumn{2}{|l|}{ Perceived Risk of Breast Cancer } \\
\hline \multicolumn{2}{|l|}{ Five-year perceived risk of breast cancer } \\
\hline Lower than an average woman & $84(15.0 \%)$ \\
\hline Similar to an average woman & $137(24.4 \%)$ \\
\hline Higher than an average woman & $65(11.6 \%)$ \\
\hline Not sure & $275(49.0 \%)$ \\
\hline Total & $561(100.0 \%)$ \\
\hline \multicolumn{2}{|l|}{ Lifetime perceived risk of breast cancer } \\
\hline Lower than an average woman & $79(13.9 \%)$ \\
\hline Similar to an average woman & $154(26.5 \%)$ \\
\hline Higher than an average woman & $81(14.3 \%)$ \\
\hline Not sure & $253(44.6 \%)$ \\
\hline Total & $567(100.0 \%)$ \\
\hline
\end{tabular}

1: Actual risk as calculated by Gail Model (1989) 
As is evident from Table 4.24, a majority of women (90.5\%) had undergone mammography screening at some point of time. However, only $50.7 \%$ of respondents reported to have had mammography screening in the past year and were thus deemed to be compliant consistent with the ACS breast cancer screening guidelines(American Cancer Society, 2002). With respect to primary prevention behavior through use of chemopreventive tamoxifen, the majority of women reported to have never taken the drug for breast cancer risk reduction.

The information provided by the respondents on their age, age of menarche, age at first live birth, family history of breast cancer, number of previous biopsies and their results was inputted into the 'Breast Risk Assessment' software (based on Gail model, 1989) developed by Cardinal Health System Inc. (Copyright 2000 C, Cardinal Health Systems Inc.) in order to obtain women's five-year relative risk of developing breast cancer. Based on the criteria used in the BCPT trial (Fischer et al., 1998) any woman below age of 60 years was deemed to be at high risk of breast cancer if her 5-year relative risk was equal to or above $1.7 \%$. Also, consistent with the BCPT trial eligibility criteria, any women who was 60 years of age or older was deemed to be at high risk of breast cancer irrespective of her actual breast cancer risk determined through the Gail model (1989). Based on these criteria, about $53 \%$ of respondents were estimated to be at high risk for breast cancer and thus eligible for chemoprevention.

Frequency runs on five-year and lifetime perceived risk revealed that most women were not sure about their 5-year and lifetime risk of developing breast cancer (49.0 \% and $44.6 \%$, respectively). This was followed by about $25 \%$ women who perceived themselves to be at a similar 5-year risk of developing breast cancer to that of an average woman and about $27 \%$ women who perceived themselves to be at a similar lifetime risk of developing breast cancer as compared to an average women. A relatively lower proportion of women perceived themselves to be at a higher risk of breast cancer (11.6\% for 5-year risk and $14.3 \%$ for lifetime risk). 
A total of fourteen questions assessed respondent's knowledge of breast cancer, its risk factors, and mammography screening. A summary of their responses is reported in Table 4.25 .

Table 4.25: Proportion of women correctly responding to knowledge items

\begin{tabular}{lc}
\hline \multicolumn{1}{c}{ Knowledge Item } & $\begin{array}{c}\text { Proportion } \\
\text { (Valid \%) }\end{array}$ \\
\hline Mammograms can detect breast lumps early & $85.0 \%$ \\
One mammogram is enough to ensure that you will not get breast cancer & $82.1 \%$ \\
Regular mammography screening reduces the risk of dying from breast cancer & $77.1 \%$ \\
$\begin{array}{l}\text { Women with close relatives with breast cancer have higher risk of breast cancer } \\
\text { Mammography can cure breast cancer }\end{array}$ & $75.6 \%$ \\
Mammography can spread breast cancer & $70.4 \%$ \\
The risk of breast cancer increases with age & $62.8 \%$ \\
$\begin{array}{l}\text { A woman currently using birth control pills has a slightly greater risk of breast cancer as } \\
\text { compared to a woman not using them } \\
\text { Long term use of estrogen replacement therapy (also known as hormone replacement } \\
\text { therapy) after menopause, slightly increases breast cancer risk } \\
\text { Breast cancer is usually painful when it is just getting started }\end{array}$ & $43.7 \%$ \\
$\begin{array}{l}\text { Breast cancers can be prevented } \\
\text { Giving birth to a first child after } 30 \text { years of age increases the risk of breast cancer }\end{array}$ & $45.3 \%$ \\
Obesity (being overweight) is a risk factor for breast cancer & $35.5 \%$ \\
\hline
\end{tabular}


For all the fourteen knowledge items, the responses were collected on a 'agree', 'don't agree', and 'don't know' scale. A 'don't know' response was deemed to be an incorrect response and was added to the incorrect response tally. In general, respondents were very knowledgeable on questions pertaining to mammography screening as is evident from Table 4.25. Eighty-five percent of the respondents believed that mammograms can detect breast lumps early and about $82 \%$ respondents agree with the fact that one mammogram was not enough to ensure secondary prevention through mammography screening. Also, a high number (about $77 \%$ ) of respondents agreed with the statements about mammography screening's ability to reduce the risk of dying from breast cancer. Respondents were least knowledgeable with respect to dietary issues such as 'alcohol consumption' and 'obesity' and its relationship to breast cancer risk with only $16.5 \%$ and $17.4 \%$ responding correctly to questions on alcohol consumption and obesity, respectively. Interestingly, a large proportion (about $70 \%$ ) of respondents reported that breast cancers can be prevented. A knowledge index was created by summing up all fourteen knowledge items and dividing the total knowledge score by fourteen (prior to adding the responses, all correct responses were coded as ' 1 ' while all other responses were coded as ' 0 '). This resulted in a knowledge index between 0 and 1 for every respondent. The standardized item alpha for the knowledge index was found to be 0.79 indicating high internal consistency. This knowledge index was then used for all further analysis.

As indicated in Table 4.24 about $53 \%$ of the respondents were at high risk of developing breast cancer. Baseline characteristics of this sub-group is presented and discussed next.

Average age of high-risk women who responded to the survey was 59.9 years (SD: 6.20 years, range: 42 years - 68 years) out of which $8.8 \%$ were 49 years of age or less, $28.1 \%$ were between 50 to 59 years of age and $63.1 \%$ were 60 years or older. Table 4.26 reports additional baseline characteristics of these high-risk women. 
Table 4.26: Characteristics of Respondents at High Risk for Breast Cancer

\begin{tabular}{|c|c|}
\hline Item/Variable & N (Valid \%) \\
\hline \multicolumn{2}{|l|}{ Breast Cancer Prevention Behavior } \\
\hline Yes & $273(92.5 \%)$ \\
\hline No & $22(7.5 \%)$ \\
\hline Total & $295(100.0 \%)$ \\
\hline \multicolumn{2}{|l|}{ How long since the last mammography } \\
\hline Less than a year & $143(54.0 \%)$ \\
\hline$>1$ year but $<2$ years & $68(25.7 \%)$ \\
\hline$>2$ years & $54(20.4 \%)$ \\
\hline Total & $265(100.0 \%)$ \\
\hline \multicolumn{2}{|l|}{ Ever taken tamoxifen } \\
\hline$\overline{\text { Yes }}$ & $2(0.7 \%)$ \\
\hline No & $287(97.6 \%)$ \\
\hline Not sure & $5(1.7 \%)$ \\
\hline Total & $294(100 \%)$ \\
\hline \multicolumn{2}{|l|}{ Perceived Risk of Breast Cancer } \\
\hline \multicolumn{2}{|c|}{ Five-year perceived risk of breast cancer } \\
\hline Lower than an average woman & $40(13.9 \%)$ \\
\hline Similar to an average woman & $58(20.1 \%)$ \\
\hline Higher than an average woman & $41(14.2 \%)$ \\
\hline Not sure & $149(51.7 \%)$ \\
\hline Total & $288(100.0 \%)$ \\
\hline \multicolumn{2}{|c|}{ Lifetime perceived risk of breast cancer } \\
\hline Lower than an average woman & $30(13.4 \%)$ \\
\hline Similar to an average woman & $72(24.8 \%)$ \\
\hline Higher than an average woman & $45(15.5 \%)$ \\
\hline Not sure & $134(46.2 \%)$ \\
\hline Total & $290(100.0 \%)$ \\
\hline
\end{tabular}


As is evident from Table 4.26, a majority of women (92.5\%) had undergone mammography screening at some point of time. Fifty-four percent of these women reported to have had mammography screening in the past year and were thus deemed to be compliant consistent with breast cancer screening guidelines of the American Cancer Society (American Cancer Society, 2002). With respect to primary prevention through use of chemopreventive tamoxifen, the majority of women (about $98 \%$ ) reported to have never taken the drug for breast cancer risk reduction.

Frequency runs on five-year and lifetime perceived risk revealed that most women were not sure about their 5-year and lifetime risks of developing breast cancer (51.7 \% and $46.2 \%$, respectively). This was followed by about $20 \%$ women who perceived themselves to be at a similar 5-year risk of developing breast cancer to that of an average woman and about $25 \%$ women who perceived themselves to be at a similar lifetime risk of developing breast cancer as compared to an average woman. A relatively lower proportion of women perceived themselves to be at a higher risk of breast cancer (14.2\% for 5-year risk and $15.5 \%$ for lifetime risk).

Table 4.27 reports responses of these high-risk women on the 14 knowledge items of breast cancer, its risk factors, and mammography screening. 
Table 4.27: Proportion of high-risk women who gave correct responses on knowledge items

\section{Knowledge Item}

Proportion

(Valid \%)

Mammograms can detect breast lumps early

$85.4 \%$

One mammogram is enough to ensure that you will not get breast cancer

$83.1 \%$

Regular mammography screening reduces the risk of dying from breast cancer

Women with close relatives with breast cancer have higher risk of breast cancer

Mammography can cure breast cancer

$67.1 \%$

Mammography can spread breast cancer

$60.3 \%$

The risk of breast cancer increases with age

$49.2 \%$

A woman currently using birth control pills has a slightly greater risk of breast cancer as compared to a woman not using them

Long term use of estrogen replacement therapy (also known as hormone replacement therapy) after menopause, slightly increases breast cancer risk

Breast cancer is usually painful when it is just getting started

Breast cancers can be prevented

Giving birth to a first child after 30 years of age increases the risk of breast cancer 
$\underline{\text { Relationships between Actual Risk of Breast cancer, Perceived Risk of Breast Cancer, }}$ Breast Cancer Knowledge, and Screening Behavior

The relationship between a respondent's actual 5-year risk (as determined by the Gail, model. 1989) and perceived 5-year risk of developing breast cancer was assessed by determining correlation coefficients between the two variables. The original 5-year perceived risk variable was re-coded to include patients who perceived themselves to be either at high or low risk of developing breast cancer. Those respondents who perceived themselves as having similar risk to an average woman or who were not sure about their 5 -year breast cancer risk were excluded from this analysis. As both the variables i.e. the perceived of breast cancer as well as actual risk of breast cancer were dichotomous in nature, 'Phi' correlation coefficient, instead of Pearson Product-Moment Correlation, was determined. A statistically significant $(r=0.167, p=0.045)$ positive correlation was observed between the perceived 5-year risk and actual 5-year risk. However, the magnitude of correlation was found to be low (Miller L, 1994) and a 'coefficient of determination' calculation indicated that only $2.8 \%$ of variance in 5 -year perceived risk is accounted for by actual risk.

Differences in knowledge index scores between high-risk respondents and their low-risk counterparts were investigated using t-tests. Interestingly, low-risk respondents were found to have significantly higher mean knowledge scores as compared to respondents who were at a high risk of developing breast cancer (low risk group $=0.54$ $( \pm 0.21)$ vs. high risk group $=0.50( \pm 0.22), \mathrm{p}=0.036)$. Further details are presented in Table 4.28.

A chi-square test was performed to assess if there was a relationship between respondent's actual risk of breast cancer and their mammography screening behavior. No significant relationship was observed between a woman's actual risk of breast cancer and her compliance with mammography screening. 
Table 4.28: Knowledge Score and Respondent's Actual Risk of Breast CanceR

\begin{tabular}{ccccc}
\hline Item & Mean $^{\dagger}( \pm$ SD $)$ & t-statistic & Df & Sig. \\
\hline Actual risk of breast cancer & & & & \\
Low risk women & $0.54( \pm 0.21)$ & -2.10 & 549.67 & $0.036^{*}$ \\
High risk women & $0.50( \pm 0.22)$ & & & \\
\hline
\end{tabular}

*significant at 0.05 level

$\dagger$ Range: 0 and 1 , where ' 0 ' indicates no correct answers and ' 1 ' indicates all correct answers

$\underline{\text { Relationships between Perceived Risk of Breast Cancer, Breast Cancer Knowledge, and }}$ $\underline{\text { Screening Behavior }}$

Relationship between respondent's 5-year perceived risk of breast cancer and her knowledge index score was assessed by means of a two-tailed student t-test. The relationship between the respondent's lifetime perceived risk of breast cancer and knowledge index was also evaluated. These results are reported in Table 4.29. Those respondents who perceived themselves to be at a high risk of developing breast cancer in the next 5 years had, on average, a significantly higher knowledge index score as compared to their counterparts who perceived themselves to be at a lower risk of breast cancer. Similarly, respondents who perceived themselves to be at a high risk of developing breast cancer in their lifetime had, on average, a significantly higher knowledge index score compared to those respondents who perceived themselves to be at a lower risk. Details are provided in Table 4.29. 
Table 4.29: Knowledge Score and Respondent's Perceived Risk of Breast Cancer

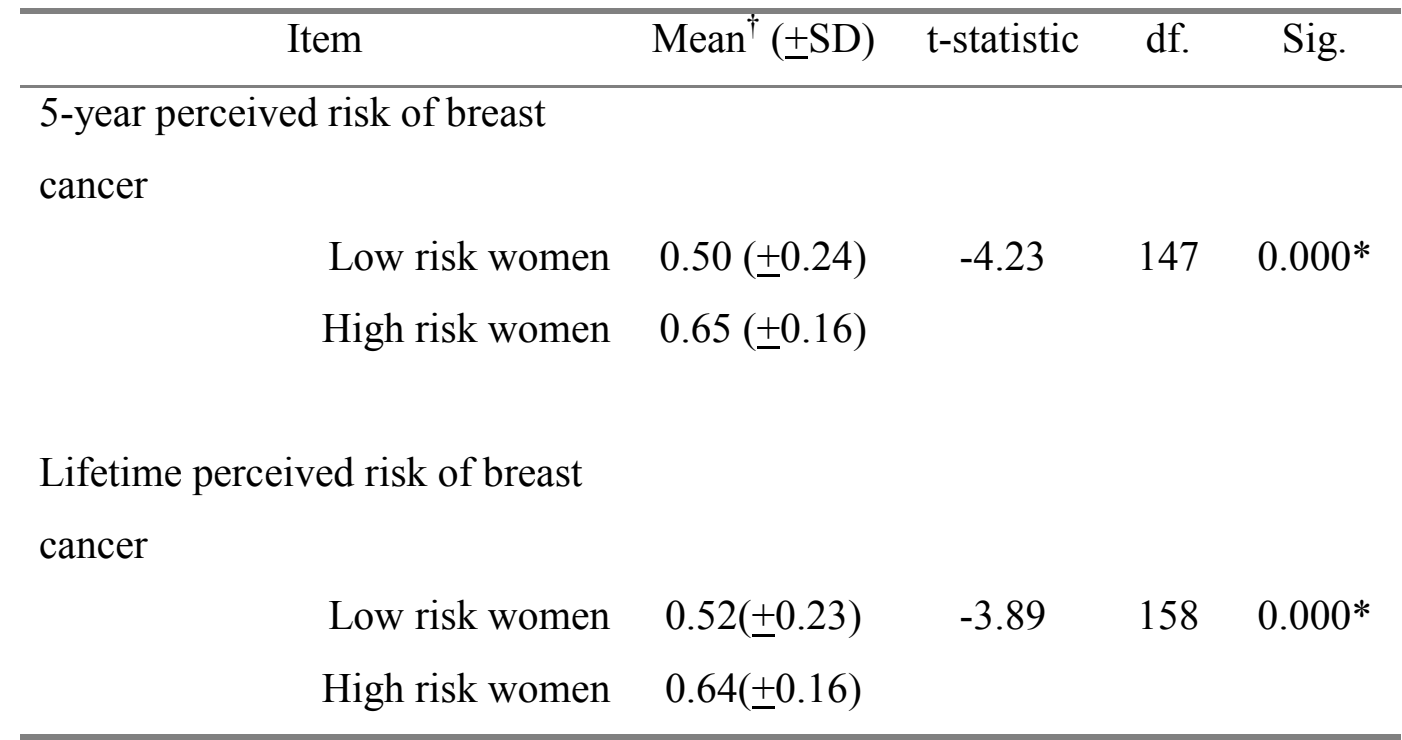

*significant at 0.05 level

$\dagger$ Range: 0 and 1 , where ' 0 ' indicates no correct answers and ' 1 ' indicates all correct answers

Next, the relationship between respondents perceived risk and mammography screening behavior was assessed. Chi-square analysis was performed in order to assess the relationship between 5-year and lifetime perceived risk and respondent's screening behavior. No significant relationship was observed between a woman's 5-year and lifetime perceived risk of breast cancer and her compliance with mammography screening.

\section{Using Chemopreventive Tamoxifen and its Relation to Actual Risk, and Perceived Risk}

Willingness to consume chemopreventive therapy among responding women revealed that a large proportion of these women $(n=236,42.3 \%)$ were not sure with respect to consuming tamoxifen for their breast cancer risk reduction. This is closely followed by those women ( $\mathrm{n}=228,40.9 \%$ ) who indicated that they would not consume chemopreventive tamoxifen even if the drug was covered by their insurance and their doctors told them that they were at a high risk of developing breast cancer. The remaining $16.8 \%(n=94)$ indicated their willingness to consume tamoxifen if their doctors told them that they were at a high risk for breast cancer and their insurance paid for the drug. 
Relationships between respondent's willingness to consume tamoxifen and their actual risk and perceived risk were assessed using chi-square tests. The results are reported in Table 4.30. No significant relationship was found between the respondent's actual risk of developing breast cancer and her willingness to consume chemopreventive tamoxifen. Similarly, there seems to be no relationship between how a woman perceives her 5-year and lifetime risk and her willingness to consume chemopreventive tamoxifen.

Table 4.30: Willingness to consume chemopreventive tamoxifen and breast cancer risk

Willingness to consume

Item

$\frac{\text { tamoxifen }}{\text { Yes }} \chi^{2}$

Actual risk of breast cancer

Low-risk

High-risk

$$
\begin{array}{ccc}
44(32.84 \%) & 90(67.16 \%) & 1.792 \\
45(25.86 \%) & 129(74.14 \%) & (\mathrm{p}=0.181) \\
& & \\
18(34.62 \%) & 34(65.38 \%) & 0.458 \\
17(41.46 \%) & 24(58.54 \%) & (\mathrm{p}=0.499) \\
& & \\
15(31.25 \%) & 33(68.75 \%) & 1.503
\end{array}
$$

5-year perceived risk of breast cancer

low-risk

high-risk

Lifetime perceived risk of breast cancer

Low-risk

High-risk 


\section{CHAPTER 5}

\section{DISCUSSION \& RECOMMENDATIONS}

Results of the BCPT trial indicated chemopreventive tamoxifen's ability to significantly reduce the risk of breast cancer in healthy women who were at a high risk of developing breast cancer (Fisher et al., 1998). Ability of mammography screening to detect breast tumors at an earlier stage thereby reducing breast cancer mortality has also been well documented (Costanza, 1992; Shapiro, 1988; Shapiro, 1989; Seidman et al., 1987; Tabar et al., 1992). However, very little is known about the effect of breast cancer risk reduction through use of chemopreventive tamoxifen on overall survival of women, especially, since the BCPT trial was not designed to detect mortality differences. Also, the BCPT trial follow-up was not sufficient to determine mortality benefits of chemopreventive tamoxifen. In addition, there is no data that estimates 'survival benefit' and 'costs' of using chemopreventive tamoxifen in presence of the more traditional breast cancer preventive strategy i.e. mammography screening. Finally, relationships between real risk of breast cancer, perceived risk of breast cancer, breast cancer knowledge, and willingness to consume tamoxifen have also not been very well documented, especially in populations like the Medicaid.

Consequently, a model, which quantifies the net costs and benefits of employing these two preventive strategies in tandem, was developed in the current study. Data were also collected prospectively by means of a mail survey to investigate the abovementioned relationships between breast cancer risk (actual and perceived), and willingness or reluctance to consume chemoprevention tamoxifen. Methodology employed in the development of the model and collection of survey data has been discussed in Chapter 3. The results of the model and the survey are reported in Chapter 4. This chapter includes a review of study findings and their implications. Major limitations of this study will be discussed, the significance of study results will be presented, and finally recommendations for future research will be made in this chapter. 


\section{Background}

Breast cancer is one of the most common types of malignancy in women, second only to lung cancer in terms of cancer-related deaths (Alberg et al., 2000). This clinical significance directly translates into substantial economic burden of approximately $\$ 6.6$ billion (1990 dollars) in treatment costs alone (about $\$ 9.7$ billion when inflated to reflect 1997 dollars) (Brown D, 1999). Until now, mammography screening has been the most effective method for preventing pre-mature mortality due to breast cancer. However, the recent approval of tamoxifen by the Food and Drug Administration (FDA) as a breast cancer risk reducing chemopreventive agent in healthy women who have high risk of developing breast cancer has provided an additional option in breast cancer prevention management.

Introduction of such preventive strategy will result in utilization of additional health care resources, which in turn will increase healthcare costs. For instance, the annual economic cost of screening in a mobile mammography program in 1998 amounted to more than $\$ 381,000$ for a low volume program and more than $\$ 789,000$ for a high volume program (Brown D et al., 1999). These costs put additional burden on third party payer systems, which are already facing resource constraints and consequently are looking for ways to reduce their expenditures. Among these third party payer systems, the Medicaid program, which is a joint federal-state program, may be one of the most susceptible systems to rising costs, especially drug costs, as it provides insurance to population that are generally in worst health than the general population and rely on Medicaid for their prescription drug coverage (Bruen B, 2002). Introducing newer prescription drugs or adding newer indications to existing drugs like tamoxifen will strain budgets of Medicaid programs which are already experiencing difficult fiscal conditions due to federal and state budget deficits (Bruen B, 2002). Consequently, there is growing pressure on state and federal governments to evaluate medical interventions that are not only effective but also economically feasible. 
The State Medicaid programs are under no obligation to cover prescription drugs in their benefit package. But once the states opt to cover prescription drugs, barring few classes of drugs, the federal law requires these states to cover all FDA-approved drugs made by manufacturers with federal rebate agreements (Bruen B, 2002). Under such federal requirements, the total prescription expenditures are bound to increase.

Moreover, some drugs like chemopreventive tamoxifen can affect total drug expenditures to a greater extent than others. Being a primary preventive drug, there is a possibility for it to be prescribed to a large proportion of women population who although are at a high risk of developing breast cancer may never go on to develop the disease. For instance, among the 6,599 high-risk women who were enrolled in the placebo arm of the BCPT trial, less than $3 \%$ women $(n=175)$ developed breast cancer (Fisher et al., 1998). The other significant cost driver in this case is the chronic nature of the chemopreventive therapy, lasting 5 years. With an annual cost of more than $\$ 1,200$ per woman, a five-year chemopreventive therapy may result in costs in excess of $\$ 6,000$ per chemopreventive therapy per woman in drug costs alone. Under such circumstances, addition of newer interventions like chemopreventive tamoxifen in addition to existing interventions like mammography screening in programs such as the Medicaid will require strict economic assessment. This in turn necessitates quantitative determination of incremental costs and consequences of newer interventions over existing ones. In absence of real world long term costs and efficacy data on chemopreventive tamoxifen, one way of quantifying these outcomes (costs and consequences) is to develop a life expectancy model, similar to the one developed in the present study. Such life expectancy models integrate data from diverse sources including randomized trials, real world observational studies etc in order to project survival benefits of an intervention.

The current study utilized results from the BCPT trial, which, at the time of this study, was the only large-scale trial in United States evaluating chemoprevention using tamoxifen. In addition to the BCPT trial there were two more trials (Powles T et al., 1998; Veronesi et al., 1995) in Europe that evaluated chemopreventive properties of tamoxifen in reducing breast cancer risk. Results from these trials, however, were not consistent with those found in the BCPT trial. Many theories were put forth to explain 
the discrepancies between the BCPT trial and the two European trials. One of them was in relation to 'power' of these European trials to detect differences seen in much larger BCPT trial. For instance, the two smaller European trials provide only slightly more than $66 \%$ of the woman-years of follow up captured in the BCPT trial. Moreover, it has been suggested that higher baseline incidence and the larger size of the BCPT trial may have been responsible for demonstrating tamoxifen's chemopreventive effect (Pritchard K, 1998). Also, there were differences in baseline characteristics of women participating the BCPT trial and the European trials in terms of their age and drug compliance profiles. Nevertheless the BCPT trial findings were argued to be robust (Pritchard K, 1998) and the decision to base the current model on BCPT trial results was driven by two main factors: (1) applicability of model results to the US population and setting, and (2) the US FDA's approval of chemopreventive tamoxifen on basis of BCPT trial results, which involved the North American population, a population similar to the one that is being considered in the current study. Results derived from this model are discussed in the following section.

\section{Review of Study Results}

A life expectancy Markov model was developed in the current study to determine the incremental costs and benefits of introducing chemopreventive tamoxifen in tandem with existing mammography screening in those women who are healthy but at a high risk of developing breast cancer. The base case model simulation, at a $3 \%$ discount rate, yielded an average incremental life expectancy gain of 0.122 years (about 45 days) in the tamoxifen plus optimal mammography screening cohort and a gain of 0.076 (about 28 days) in the tamoxifen plus non-optimal mammography screening cohort as compared to only-mammography screening cohort. This incremental gain of 0.122 life years and 0.076 life years came at a cost of $\$ 5,969.68$ and $\$ 4,916.84$ (2001 dollars) resulting in an ICER of $\$ 48,932$ and $\$ 64,695.20$ per life year gained, respectively.

As this study was the first study to evaluate the combination effect of chemopreventive tamoxifen and mammography screening, results from this study were not directly comparable to other studies. However, a literature review identified studies 
that had investigated costs and benefits of chemopreventive tamoxifen alone compared to no therapy in high-risk women and although not directly comparable to the present study they did provide a benchmark against which to compare the current results. A study by Noe et al., (1999), demonstrated that high-risk women who begin consuming chemopreventive tamoxifen between ages of 35 to 49 years, experience on average, a life expectancy gain of 0.1028 years (about 38 days) at $3 \%$ discount rate. This life expectancy gain is comparable to the gain of 0.122 years (about 45 days) found in the current study. The ICER for cost per life years gained in the Noe et al., (1999) study was reported to be $\$ 41,372$ which again was comparable to the ICER of $\$ 48,932$ per life year gained determined in the current study. The slightly higher gain in life expectancy and higher costs in the current study can be, in part, attributed to the fact that the effects (both costs and benefits) of mammography screening were modeled in the current study unlike the Noe et al., (1999) study, which compared chemopreventive tamoxifen alone to no therapy.

A second study (Grann et al., 2000), which compared incremental costs and benefits of chemopreventive tamoxifen alone to no therapy, estimated the survival benefits of 69 days (about 0.189 years). Slight differences can be once again attributed to different interventions being investigated in the Grann et al., (2000) study (chemopreventive tamoxifen vs. no therapy) and the current study (chemopreventive tamoxifen plus mammography screening vs. mammography screening alone) and also on basis of starting age of cohort which was 35 years in Grann et al., study as opposed to 40 years in the current study. For instance, Grann et al., (2000) found a diminished survival benefit of 40 days in women who started on chemopreventive tamoxifen at 50 years of age and a survival benefit of 27 days in women who started the therapy at 60 years of age. The incremental cost per life years gained of $\$ 46,619$ found in the Grann et al., (2000) study was also found to be comparable to $\$ 48,932$ per life year gained determined in the current study.

Although, it is difficult to establish a benchmark for cost-effectiveness, in general, any strategy resulting in less than $\$ 50,000$ per life year saved is considered to be cost- 
effective while anything above $\$ 100,000$ is considered excessive (Mark et al., 1995). However, a significant number of interventions that cost as much as $\$ 100,000$ per-life year saved or even more have been accepted in clinical practice (Hillman et al., 1995). Based on the current model, chemopreventive tamoxifen in presence of optimal mammography screening resulted in a incremental cost-effectiveness ratio of $\$ 48,932$ per life year saved which is slightly below the cost-effectiveness "threshold" of $\$ 50,000$ per life year saved. This incremental cost effectiveness ratio increased to $\$ 64,695.20$ per life year gained when mammography screening rates were adjusted to reflect 'real-world' rates. As was evident from the sensitivity analysis tables and graphs in Chapter 4, these base case values of $\$ 48,932$ and $\$ 64,695.20$ per life year gained fluctuated with variation in input parameters and in some instances approached the threshold value of $\$ 100,000$. For instance, a $20 \%$ decrease in tamoxifen efficacy in reducing breast cancer risk resulted in incremental cost-effectiveness ratio of $\$ 94,537$ per life year gained in the tamoxifen plus non-optimal mammography screening cohort. However, despite these variations in input parameters over a relatively wide range of values $(20 \%$ from base case), the incremental cost-effectiveness ratio for both the tamoxifen cohorts remained below $\$ 100,000$ per life year saved suggesting economic viability of tamoxifen as a chemopreventive agent.

The basic principles of cost-effectiveness analysis in health care are that resources in health sector should be allocated across the interventions and population groups so as to generate highest possible health benefit through fixed resources (Murray C. et al., 2000). Thus, by moving resources from cost-ineffective interventions to more costeffective ones, similar or more amount of health benefits can be achieved with greater efficiency (i.e. similar or more benefits at lesser cost). Economic evaluations, although have a clear role in healthcare resource allocation decisions, they do not by themselves dictate resource allocation decisions. For instance, reimbursement and formulary approval agencies have started questioning the coverage of certain medicines on many instances despite their favorable cost-effectiveness profiles (Trueman et al., 2001). This reluctance to accept newer interventions purely on their cost-effectiveness profiles is based on the reasoning that despite the goal of economic analysis is to maximize 
efficiency these agencies have to function within a constrained budgets (Trueman et al., 2001).

For instance, in the United Kingdom, the Standing Medical Advisory Council (SMAC) published guidelines on 'statin' use among certain groups of patients who would benefit most thereby prioritizing use of the drug to those individuals who had a high risk of developing coronary heart disease or those individuals who had experienced myocardial infarction in the past (Standing Medical Advisory Council, 1997; Trueman et al., 2001). Based on these criteria, it was estimated that about $8.2 \%$ of the population would be eligible to receive the statins. Despite the risk based 'selective' approach of the guidelines, it was estimated that adherence to these guidelines would result in a $20 \%$ increase in annual drug budget of the involved parties (Trueman et al., 2001). Later, a study (Primatesta P et al., 2000) found that prescribing of statins, despite their significant clinical effectiveness as demonstrated by numerous clinical trials (Scandinavian Simvastatin Survival Group, 1994), was significantly below the recommended levels. This discrepancy between prescribing levels and recommended level was thought to be related to substantial costs of providing treatment consistent with the guidelines (Trueman et al., 2001) highlighting the distinction between being effective and being affordable. In other words, being cost effective and thus being efficient does not automatically translate into being affordable. As is clearly evident from the above example, affordability of an intervention in a given health care setting is not only dictated by its cost-effectiveness but also by the extent to which that intervention is accepted and utilized in that setting. Thus, in the present case, ability of WVMP to afford chemopreventive tamoxifen in addition to ongoing mammography screening, will not only depend on tamoxifen's cost-effectiveness profile, which was already demonstrated in phase I of this study, but also on total number of women in the program who are likely to consume the drug. This lack of information on proportion of women in the WVMP who can be potential consumers of chemopreventive tamoxifen and thus partly affect its affordability led to development of phase II of this study. 
One of the primary objectives of phase II was to estimate the proportion of women in the WVMP who were at a high risk of developing breast cancer and were thus eligible for chemoprevention through use of tamoxifen. A woman's individualized risk of developing breast cancer was assessed by means of a mail survey to randomly selected women who were enrollees of the WVMP. Information obtained from the survey was then inputted into the Gail model in order to obtain the 5-year breast cancer risk. Based on the BCPT trial guidelines (Fisher et al., 1998), all women who were 60 years of age and above or were between ages of 40 to 59 years (40 and 59 included) and had a 5 -year breast cancer risk $\geq 1.7 \%$ were deemed high risk women. Data analysis based on survey responses revealed that about $53 \%$ of respondents were at a high risk of developing breast cancer.

The WVMP in the year 1999 (the year from which the study sample was derived) had a total of 35,657 female enrollees who were greater than 40 years of age. Out of these 2,451 enrollees were part of Medicaid-managed care and were excluded from further analysis as costs incurred to these women may not be reimbursed by the program directly. Extrapolating the results from the current survey to West Virginia Medicaid enrollee population revealed that an estimated 17,600 women may be at high risk of developing breast cancer and thereby eligible to receive chemopreventive tamoxifen. However, eligibility for an intervention does not automatically ensure its acceptance. Stated otherwise, the use of chemopreventive tamoxifen in an otherwise healthy population may not be purely based on baseline risk of the individual or population but rather on a thorough risk-and-benefit analysis of the drug in terms of its protective antiestrogenic effects and harmful estrogenic effects (Bastian L et al., 2001). This was apparent when the respondents in the current survey were asked about their willingness to consume tamoxifen if they had no financial risk with respect to cost of the drug and that their doctor had told them that they were at a high risk of developing breast cancer. Only about $17 \%$ respondents said that they would be willing to take the drug under such circumstances. This finding was slightly lower than the one reported in a previous study by Bastian et al., (2001) where about $23 \%$ of surveyed women showed inclination towards using chemopreventive drugs. This can be explained in part on the basis that 
Bastian et al., (2001) assessed women's interest in chemoprevention without providing information on potential risks of chemopreventive drug. In the current study, however, the surveyed women were sensitized to the issue of serious adverse events that may occur as a result tamoxifen, which may have resulted in relatively lower willingness to consume the drug.

Since many women may not be aware of their actual risk of developing breast cancer (Evans D et al., 1993; Cull A et al., 1999), it is very likely that acceptance or reluctance towards chemopreventive drugs will be driven by their perceived susceptibility towards breast cancer rather than their actual risk of breast cancer (Bastion et al., 2001). Results from the current study were consistent with those observed in the Bastion et al., (2001) study. Thus, when 'willingness to consume chemopreventive tamoxifen' was assessed based on actual breast cancer risk-stratification (into higher and lower breast cancer risk) no significant relationship was observed. Interestingly, no relationship was observed between willingness to consume the drug and woman's perceived 5-year and lifetime risk, which was contrary to what was observed in the Bastion et al., (2001) study.

It has been frequently reported in the literature (Woloshin et al., 1999; Hopwood, 2000) that women's breast cancer risk perceptions are in variance with medical perspectives. This is consistent with a positive but low correlation $(r=0.167, p=0.045)$ found between woman's actual risk and perceived risk in the current study. Moreover, studies indicate that some women significantly over-predict their breast cancer risk while others under-predict their risk. More specifically, younger women have been found to overestimate their breast cancer risk (Lee J, 1993; Eddy D et al., 1988; Baines C, 1992). This was observed in the current study in which women who perceived themselves at high risk (for both 5-year and lifetime risk) were significantly younger than their counterparts who perceived themselves at a lower risk.

The current study also investigated the relationship between breast cancer risk and compliance with mammography screening. No significant relationship was observed between woman's actual or perceived risk of breast cancer and her mammography 
screening behavior. Results from published studies that have evaluated relationships between women's screening behavior and their breast cancer risk have found contradictory results with some studies reporting positive relationship while others reporting no relationship (Carney P et al., 2002; Beaulieu M et al., 1996; Cole S et al., 1997; Aiken L et al., 1995).

So far, the results from phase I and phase II of this study have been presented in isolation from each other as they represent different aspects of prevention management. Phase I dealt with quantifying costs and consequences of introducing chemopreventive tamoxifen in addition to more traditional mammography screening in women who were healthy but at high risk of developing breast cancer. These costs and consequences, however, were determined on per woman basis. Phase II of the study was an extension of phase I in that it determined proportion of population that was eligible to receive chemopreventive tamoxifen thereby allowing estimation of total costs and benefits to the entire health system, in this case the WVMP. This impact of introducing chemopreventive tamoxifen in WVMP is discussed next.

\section{Implication of Study Findings}

Results from the life expectancy model in phase I of the current study indicate that high risk women who start on chemopreventive tamoxifen at age 40 will experience an average (per patient) a life expectancy gain of 0.122 years (about 45 days) if they undergo routine mammography screening or experience a gain of 0.076 years (about 28 days) if they continue mammography screening at currently observed rates. Incorporating costs in this model indicated that the women in the two tamoxifen cohorts would also incur an average incremental cost (over and above what is incurred to women in non-tamoxifen group) of $\$ 5,969.68$ and $\$ 4,916.84$, respectively. Phase II of the study indicated that about $53 \%$ of the surveyed WVMP women were at high risk of developing breast cancer and thereby eligible for chemopreventive tamoxifen. Based on an analysis presented earlier in this chapter, a total of about 17,600 WVMP women enrollees can be expected to be at high risk of developing breast cancer. 
If every woman in the WVMP, who was eligible to receive chemopreventive tamoxifen, started consuming the drug at age 40 years, there will be a total life expectancy gain of 2,147 life years (per woman life expectancy gain of 0.122 years * eligible population of 17,600 women) in the entire eligible population if everyone underwent routine mammography screening. This increase in total life expectancy gain of 2,147 life years will come at a total cost of about \$105 million (incremental cost of $\$ 5,969.68$ per woman * eligible population of 17,600 women). A relatively lower gains in life expectancy of 1,338 years (per woman life expectancy gain of 0.076 years * eligible population of 17,600 women) will be achieved if all women in the WVMP consume chemopreventive tamoxifen but undergo mammography screening at currently observed sub-optimal rates. This increase in total life expectancy gain of 1,338 life years will come at a total cost of about $\$ 87$ million (incremental cost of $\$ 4,916.84$ per woman * eligible population of 17,600 women).

An issue in direct extrapolation of costs and benefits is acceptability of intervention irrespective of its efficacy. As discussed earlier, eligibility for a given intervention does not directly translate into its acceptance in the desired population. As was evident in the current as well as an earlier study (Bastion et al., 2001) very few women expressed interest in chemopreventive tamoxifen despite its clinical efficacy in breast cancer risk reduction. Results from the mail survey in the current study estimated that only about $17 \%$ of women would consume tamoxifen given its risks and benefits. Hence, a more appropriate extrapolation in the present case may be to include only $17 \%$ of the eligible population as opposed to the entire population. Based on this reasoning, the total number of the WVMP enrollees that may get chemopreventive tamoxifen is estimated to be about 2,992 women. Hence, life expectancy gains as well as costs were recalculated for this sub-set of women who showed positive inclination of using tamoxifen in breast cancer chemoprevention. This scaled down estimate of baseline population indicated that introduction of chemopreventive tamoxifen in the WVMP will result in a gain of about 365 life years at an additional total cost of approximately $\$ 18$ million in a population which undergoes routine mammography screening. At currently observed mammography screening rates, introduction of chemopreventive tamoxifen in 
the WVMP will result in a gain of about 227 life years at an additional total cost of approximately $\$ 15$ million.

As is evident from the above calculations, acceptance rate of chemopreventive tamoxifen within a population has a significant impact on its total costs and benefits in that population. These acceptance rates may vary across different populations due to differences in baseline characteristics, cultural differences, susceptibility towards breast cancer, perceived risk, and knowledge among other factors. Very limited knowledge exists with regards to this issue due to the fact that chemopreventive tamoxifen is a relatively recent phenomenon. The current study provides preliminary data on primary preventive behavior of women, which needs to be explored further in more details to be able to make better estimation of tamoxifen's impact on a particular system. This is one of the limitations of the current study and is discussed in more details along with other study limitations in the next section.

\section{$\underline{\text { Study Limitations }}$}

Following is a list of limitations that the current study possesses. Some of these limitations may affect the results and their interpretability to a greater extent than others. Nevertheless, these limitations need to be considered when deriving inferences from the reported results.

1. The implicit assumption made in the current study is that results from the BCPT trial are generalizable and thus are applicable from one population setting to another.

Differences in study results between the US based BCPT trial and the two European trials suggest that there may be population differences in terms of response to chemopreventive tamoxifen. However, many theories have been proposed, mostly statistical, which downplay the effect of different populations on inconsistent results. The current study was performed assuming a US perspective and BCPT trial was a population based large randomized trial involving multiple centers across North America ascertaining its broad applicability, at least within the US.

2. A major issue with chronic therapy is that of drug compliance. This sub-optimal compliance expected with chemopreventive tamoxifen was modeled in the current study. 
However, the assumption that was made for modeling compliance is that women who were considered compliant consumed $100 \%$ of their drug while the remaining noncompliant women consumed no drug at all. In reality, consumption of tamoxifen by women can be expected to be along a continuous scale from low compliers to high compliers rather than a dichotomous relationship that was used in the current model. The main reason for adopting the latter approach is complete lack of information on relationship between amount and duration of drug consumed and relative magnitude of its effect. In absence of this information, it was better to model compliance using the dichotomous approach rather than assuming a highly unlikely scenario wherein all women would be compliant with tamoxifen over the 5-year therapy period.

3. As was evident from the analysis conducted in an earlier section of this chapter, the degree of acceptance of an intervention in a given population will have a significant effect on both the costs and consequences of that intervention in that population. In the current study, the interest in taking chemopreventive tamoxifen was assessed by selfreporting as opposed to using a observational database or validated behavioral models. Chemopreventive tamoxifen, being a fairly recent phenomenon, it is highly unlikely for compliance information to be available through observational databases nor are there any validated models in the literature that predict compliance with this drug or other chemopreventive drugs. Hence, there is a strong need for further research to get more robust data on patient acceptance of certain interventions. Till then, simplistic estimates derived from self-reported data can serve as a benchmark to assess acceptance/reluctance towards newer interventions an its subsequent impact on the health system.

4. One of the tamoxifen intervention arms in the current model assumes $100 \%$ compliance with screening mammography. This assumption was necessary so as to be able to fully quantify the costs and consequences of introducing chemopreventive tamoxifen under ideal mammography screening conditions versus existing sub-optimal use of mammography screening that was generally observed in a given population. However, the model in its current form does not incorporate the costs of enhancing mammography rates from sub-optimal levels to $100 \%$ compliance level. The reasoning behind not including these costs was significant amount of indirect and intangible costs and behavioral confounders, which may not be directly pertinent to the Medicaid 
program. However, despite the limitations, a simplistic analysis was performed based on estimates from a study which examined cost-effectiveness of five combinations of interventions such as physician recommendation, and telephone and in-person individualized counseling strategies for increasing compliance with mammography (Saywell R et al., 1999). This study estimated cost per every percent increase in mammography screening rate and reported that telephone plus letter was most costeffective approach with $\$ 0.78$ per one $\%$ increase in mammography rate. For the current analysis, these estimates were inputted in the model. In the non-tamoxifen cohort, a rate of $46.7 \%$ was used for defining mammography screening compliance rate. Thus, based on Saywell et al., (1999) mammography compliance cost-effectiveness study, it will cost about $\$ 42$ per woman (cost per $1 \%$ increase in mammography screening rate of $\$ 0.78 *$ percentage increase needed to achieve $100 \%$ compliance) to increase mammography rates to $100 \%$. Inputting this value in the tamoxifen cohort of the model resulted in a incremental cost-effectiveness ratio of about $\$ 55,580$ per life year saved.

5. The phase II of this study was a mail survey and therefore all limitations inherent to mail surveys will be associated with this study as well. One of the major limitations that may affect this study is recall bias, which will affect the accuracy of inferences and extrapolations, based on survey responses.

6. Another important issue associated with mail survey is that of a low response rate. It is quite possible that women who did not respond were different than those who did in terms of their attitudes, behaviors, and baseline characteristics. A non-response survey, which followed the three survey mailings, indicated that the non-respondents were similar to respondents in terms of their age and risk profile, thereby addressing the issue of non-response bias to some degree.

\section{$\underline{\text { Recommendations for Future Research }}$}

A model is only as good as the data that is used to develop that model. Chemoprevention through use of tamoxifen is a recent phenomenon and most of the clinical data that currently exists is based purely on randomized trials, which are conducted in a very controlled setting. Such data provides more information on the 'efficacy' of the drug more than its 'effectiveness.' Future research should be targeted at 
developing models, similar to the one developed in the current study, based on more real world data. Although, many precautions were taken in the current model to make the results more generalizable, for instance, by means of factoring drug non-compliance, including population based transition rates for mortality determinations etc., there is no substitute for real world data which may be only available at a later point of time.

The present analysis compared chemopreventive tamoxifen in addition to more traditional mammography screening to mammography screening alone. Similar agents with much improved side effect profiles such as raloxifene are being currently investigated for their role in breast cancer chemoprevention. One of the main issues in incorporating raloxifene intervention in the current study was that raloxifene has never been evaluated in premenopausal high-risk women (Jordan V et al., 2002). Thus future research should investigate incremental costs and consequence of agents like raloxifene to determine which agents deliver most benefits at least cost.

As is evident from the discussion, the total impact of an intervention does not only depend on its clinical and cost-effectiveness profile but also on its general acceptance in the population in which the intervention is being targeted. In the current study, willingness of women to consume chemopreventive tamoxifen for reducing their risk of breast cancer was assessed. Although, these women were made aware of potential benefits and risks of the drug, it still was a simplistic approach lacking any established behavioral theories or model. Complicating this matter is the fact that there is extreme lack of "willingness or reluctance to consume" data in the literature, which can substantiate the current study findings. Also, it is too early to predict the inclination of population towards consuming such drug as these agents have been a fairly recent phenomenon. It is quite possible that inclination towards using such agents may sway either way once these agents become routinely available in clinical practice. Future research should be directed at addressing some of the limitations that were inherent in the current study. Secondary and observational databases in the future should be evaluated to assess 'real world' consumer acceptance of such chemopreventive interventions. Also, behavioral models need to be developed, which can better predict an individual's 
acceptance/reluctance towards such agents. Once more data (through behavioral approach and 'real world' database analysis) are available to accurately predict who will accept chemoprevention and who will not, cost-effectiveness models like the one developed in the current study, can be reapplied in those situations to obtain more precise estimates of impact of interventions to third party payers and insurance systems like the Medicaid program. 


\section{Conclusions}

Tamoxifen coupled with mammography screening prolonged the average survival of cohort members who started consuming the drug at age forty as compared to the mammography screening only group. Among the two tamoxifen cohorts, average survival was found to be higher in the group which underwent routine mammography screening as compared to the group that underwent mammography screening at rates observed in real-world setting. In terms of cost, the tamoxifen cohort which underwent routine mammography screening incurred the highest cost followed by tamoxifen cohort which underwent screening at observed rates. The mammography-screening cohort incurred the least cost. Results from the univariate and bivariate sensitivity analysis (which involved varying intervention-related effectiveness parameters, rates of intervention-related adverse events, compliance with intervention, and mortality rates associated with different health states in the model) indicated that for all variations in input parameters, tamoxifen plus mammography screening (both optimal rates and observed rates) had an incremental cost-effectiveness ratio that was within the accepted threshold of $\$ 50,000$ to $\$ 100,000$ per life years saved as compared to mammography screening only. About half of the surveyed population was at a high risk of developing breast cancer and thus eligible to receive chemopreventive tamoxifen. However, only about $17 \%$ of the respondents indicated their inclination towards consuming the drug for reducing their risk of breast cancer. A statistically significant but low correlation was observed between the respondent's perceived risk and real risk indicating the need for increasing breast cancer awareness. No significant relationship was observed between a woman's risk of breast cancer, either actual or perceived, and her compliance with mammography screening. Respondent's actual risk of developing breast cancer did not appear to have any relationship with willingness to consume chemopreventive tamoxifen. This supports the need for enhancing breast cancer awareness. Interestingly, no relationship was observed between how a woman perceives her breast cancer risk and her willingness to consume chemopreventive tamoxifen. In summary, tamoxifen plus mammography screening in high-risk women is a cost-effective strategy. However, at 
present, very few of these high-risk women may actually utilize the intervention.

Moreover, additional research is needed to understand women's behaviors and attitudes in relation to chemoprevention. 


\section{REFERENCES}

Aiken L, West S, Woodward C, Reno R, Health-beliefs and compliance with mammography-screening recommendations in asymptomatic women. Health Pschol $1994 ; 12: 122-129$

Aiken L, Fenaughty A, West S, Johnson J, Luckett T. Perceived determinants of risk for breast cancer and the relations among objective risk, perceived risk, and screening behavior over time. Womens Health 1995 Spring;1(1):27-50

Alberg A, Singh S, May J, Helzlsouer K. Epidemiology, prevention and early detection of breast cancer. Cur Opin Oncol 2000; 12: 515-520

American Cancer Society. Breast Cancer Facts and Figures, Atlanta, Georgia. 2001-2002

American Cancer Society, Breast Cancer Facts and Figures, Atlanta, Georgia. 2000

American Cancer Society: Report of the Workshop on Guidelines for Breast Cancer Detection, Atlanta, GA, American Cancer Society, March 1997

American Society of Clinical Oncologist (www.asco.org/people/cn/html/m_cn998burstein.htm)

Anderson D, Badzioch M, Risk of familial breast cancer, Cancer, 1985; 56:383-387

Anderson I, Aspergen K, Janzon L et al., Mammographic screening and mortality from breast cancer; The Malmö mammographic screening trial, BMJ 1988;297:943-948

Babbie E (Ed.) (1989). The practice of social research (5th Edition). Belmont, CA: Wadsworth

Baines J Women and breast cancer: is it really possible for public to be well informed? Cab Ned Assoc J 1992; 146:2147-2148

Baker L, "Breast cancer detection demonstration project: Five year summary report", Cancer 1982; 32: 194-225

Baker $\mathrm{M}$ et al., Estimating the treatment costs of breast and lung cancer, Medical Care 1991;29(1):40-49

Barton $\mathrm{M}$ et al., The Screening Clinical Breast Examination: Should It Be Done? How?, JAMA. 1999;282:1270-1280

Bastian L, Lipkus I, Kuchibhatla M et al., Womens's interest in chemoprevention for breast cancer. Archives of Internal Medicine. 2001;161(13):1639-1644 
Beaulieu M, Beland F, Roy D, Falardeau M, Hebert G. Factors determining compliance with screening mammography. CMAJ 1996 May 1;154(9):1335-43

Beck J, Pauker S, The Markov process in medical prognosis, Medical Decision Making, 1983; 3(4): 419-458

Bjurstam N, Bjorneld L, Duffy S, Smith T, Cahlin E, Erikson O, et al., The Gothenburg Breast Cancer Screening Trial. Monogr Natl Cancer Inst 1997;22:53-5

Black W, Nease R, Tosteson A, Perceptions of breast cancer risk and screening effectiveness in women younger than 50 years of age, Journal of the National Cancer Institute, 1995;87(10):720-731

Briggs A, Sculpher A, Buxton, M. Uncertainty in economic evaluation of health care technologies. Health Economics 1994; 3:95-104

Briggs A, Sculpher M, An introduction to Markov modeling for economic evaluation, Pharmacoeconomics 1998;13(4):397-409

Brook R, Park R, Chassin M, Solomon D, Keesey J, Kosecoff J. Predicting the appropriate use of carotid endarterectomy, upper gastrointestinal endoscopy, and coronary angiography. New England Journal of Medicine 1990; 323:1173-7

Brown D, French M, Schweitzer M, McGeary K, McCoy C, Ullman S. Economic evaluation of breast cancer screening. Cancer Practice 1999; 7(1): 28-33

Brown M, Houn F, Sickles E, Kessler L. Screening mammography in community practice: positive predictive value of abnormal findings and yield of follow-up diagnostic procedures. AJR AM J Roentgenol. 1995;273:1373-7

Bruen B. States strive to limit Medicaid expenditures for prescribed drugs. Kaiser Commission on Medicaid and the Uninsured. 2002

Burke W, Daly M, Garber J, Botkin J, Kahn MJ, Lynch P, et al. Recommendations for follow-up care of individuals with an inherited predisposition to cancer. II. BRCA1 and BRCA2. Cancer Genetics Studies Consortium. JAMA 1997; 277: 997-1003.

Bush $\mathrm{T}$ et al., Tamoxifen for the primary prevention of breast cancer, Epidemiologic Reviews 1993, 15(1): 233-243

Calle E, Flanders W, Thun M, Martin L, Demographic predictors of mammography and pap smear screening in US women, American journal of Public Health, 1993;Vol. 83, No. $1: 53-60$

Cardinal Health Systems Inc. Copyright 2000. 'Breast Risk Assessment' program 
Cardinale B: Drug Topics Red Book, Pharmacy Fundamental Reference, Montvale, NJ: Medical Economics Company, 1998

Carney P, Harwood B, Weiss J, et al., Factors associated with interval adherence to mammography screening in a population-based sample of New Hampshire women. Cancer 2002 Jul 15;95(2):219-27

Centers for Medicare \& Medicaid Services, Department of Health and Human Services (DHHS) (http://cms.hhs.gov/about/)

Claus E, Risch N, Thomson W, Autosomal dominant inheritance of early-onset breast cancer: Implications for risk prediction, Cancer 1994; 73:643-651

Cole S, Bryant C, McDermott R, Sorrell C, Flynn M. Beliefs and mammography screening. Am J Prev Med 1997 Nov-Dec;13(6):439-43

Copyright (C) 2002, SPSS Inc. All rights reserved. SPSS Inc. Headquarters, 233 S. Wacker Drive, 11th floor Chicago, Illinois 60606

Costanza M, Breast cancer screening in older women, Cancer 1992;69(7 suppl): 19251931

Cull A, Anderson E, Campbell S et al., The impact of genetic counseling about breast cancer risk on women's risk perceptions and levels of distress. Br J Cancer 1999, 79:501508

Cyrus-David M, Strom S, Chemoprevention of breast cancer with selective estrogen receptor modulators: view from broadly diverse focus groups of women with elevated risk of breast cancer. Psycho-Oncology 2001;10: 521-533

Davis T, Arnold C, Berkel H, Nandy I, Jackson R, Glass J, Knowledge and attitude on screening mammography among low-literate, low income women, Cancer 1996;

78:1912-1920

Dupont W, Page D, Risk factors for breast cancer in women with proliferative breast disease, New England journal of Meedicine, 1985; 312: 146

Early Breast Cancer Trialists' Collaborative Group. Tamoxifen for early breast cancer: An overview of the randomized trials. Lancet 1998;351:1451-1467

Eddy D, Hasselblad V, McGivney W et al., The value of mammography screening in women under age 50 years. JAMA 1988; 259:1512-1519 
Elwood J, Cox B, Richardson A, The effectiveness of breast cancer screening by mammography in younger women, Online J Curr Clin Trials (serial online), 1993;2 Doc NR 32

Evans D, Burnell L, Hopwood P, Howell A. Perception of risk in women with a family history of breast cancer. Br J Cancer 1993, 67:612-614

Feig S. Mammographic screening of women aged 40-49 years. Benefit, risk, and cost considerations. Cancer. 1995;76:2097-2106

Fisher B, Costantino J, Wickerham D, Redmond C, Kavanah M, Cronin W et al., Tamoxifen for prevention of breast cancer, Journal of the National Cancer Institute 1998; 90(18):1371-1388

Fletcher S, Black W Hrris R et al., Report of the International Workshop on Screening for Breast Cancer. J Natl Cancer Inst 1993;85:1644-1656

Frisell J, Glas U, Hellstrom L, Somell A. Randomized mammographic screening for breast cancer in Stockholm. In Breast cancer research and treatment. Boston: MartinusNijhoff, 1986;8:45-54

Gail M, Benichou J, Assessing the risk of breast cancer in individuals, In: De Vita VT Jr, Hellman S, Rosenberg SA, Eds. Cancer Prevention, Philadelphia, PA: Lipincott; 1992:115

Gail M, Brinton L, Byar D, Corle D, Green S, Schairer C, et al., Projecting individualized probabilities of developing breast cancer for white females who are being examined annually, Journal of National Cancer Institute. 1989; 81:1879-1886

Garfinkel L, Boring CC, Heath C. Changing trends: An overview of breast cancer incidence and mortality. Cancer 1994; 74: 222-227

Gastrin $\mathrm{G}$ et al., Incidence and mortality from breast cancer in the Mama Program for breast screening in Finland, 1973-1986 Cancer 1994;73:2168-74

Glasziou P, Woodward A, Mahon C, Mammographic screening trials for women aged under 50. A quality assessment and meta analysis. Med J Aust. 1995; 162: 625-9

Gold M, Siegel J, Russell L, et al., Cost effectiveness in health and medicine. New York: Oxford University Press, 1996: 248

Gompertz B, On the nature of the function expressive of the law of human mortality. Philos Trans R Soc Lond 1825;115:513-585 
Grann V, Sundararajan V, Jacobson J, Whang W, Heitjan D, Antman K et al., Decision analysis of tamoxifen for the prevention of invasive breast cancer, The Cancer Journal, 2000;6(3):169-178

Harris R, Fletcher S, Gonzalez J, Lannin D, Degnan D, Earp J et al., Mammography and Age: Are We Targeting the Wrong Women, Cancer, 67, April 1991: 2010-2014.

Hartmann L, Schaid D, Woods J, Crotty T, Myers J, Arnold P et al., Efficacy of bilateral prophylactic mastectomy in women with a family history of breast cancer. NEJM 1999;340(2):7784

CMS (Health Care Financing Administration), Biennial mammography screening rates for 1998-1999 Final Report, Health Economic Research, Inc.

Henderson C., "Risk Factors for Breast Cancer Development", Cancer Supplement, March 15,1993, Volume 71, No.6

Hendrick R et al., Benefit of screening mammography in women aged 40-49 years. A new meta-analysis of randomized controlled trials. Monogr Natl Cancer Inst 1997;22:8792

Hershman D, Sundararajan V, Jacobson J, Heitjan D, Neugut A, Grann V. J Clin Oncol 2002, 20:9-16

Hicks M, Davis J, Layton J, Present A. Sensitivity of mammography and physical examination of breast for detecting breast cancer, Journal of American Medical Association, 1979; 242: 2080-2083

Hillman A, Kim M. Economic decision making in healthcare: A standard approach to discounting health outcomes. Pharmacoeconomics. 1995;7:198-205

Hopwood P, Breast cancer risk perception: what do we know and understand? Breast Cancer research 2000;2:387-391

Hunter D et al., Cohort studies of fat intake and risk of breast cancer, NEJM 1996;334(6):256-361

Hunter D et al., Plasma organochlorine levels and risk of breast cancer. NEJM 1997;337(18):1253-1258

Jordan V, Morrow M, Chemoprevention of breast cancer: a model for change. J Clin Oncol 2002 Jan 1;20(1):1-3 
Jordan V, Allen K. Evaluation of anti-tumor activity of the non-steroidal anti-estrogen monohydroxytamoxifen in the DMBA-induced rat mammary carcinoma model. Eur J Cancer 1980;16:239-51

Kalton G (Ed.) (1987), Introduction to survey sampling, Sage University Paper series on Quantitative Application in Social Sciences, Series/Number 07-035. Beverly Hills and London: Sage Pubns.

Kelloff G, Crowell J, Steele V, et al., Progress in cancer chemoprevention. Annals of The New York Academy of Sciences, 1999;Vol. 889:1-13

Kelloff G et al., Progress in clinical chemoprevention. Semin. Oncol. 24; 1997(a): 241252

Kelloff $\mathrm{G}$ et al., Risk biomarkers and current strategies for cancer chemoprevention. J. Cell. Biochem. Suppl. 25; 1997(b): 1-14

Kerlikowske K, Efficacy of screening mammography among women aged 40 to 49 years and 50 to 69 years: comparison of relative and absolute benefit. Monogr Natl Cancer Inst. 1997; 22: 79-86

Kerlikowske K, Grady D, Rubin S, Sandrock C, Ernster V, Efficacy of screening mammography- A meta analysis. JAMA 1995; 273:149-54

Kielhorn A, Graf van der Schulenburg. The health economics handbook, II edition 2000, Adis International Limited, Chester, England

Kopans D, Breast Imaging, 2nd edition, Leppincott-Raven Publishers, Philadelphia, 1998

Krieger $\mathrm{N}$ et al., Breast cancer and serum olganochlorine residues, J Natl Cancer Inst 1994, 86(8):589-599

Kvale G, Heuch I, Menstrual factors and breast cancer risk, Cancer, 1988;62: 1652-1631

Lee J. Screening and informed consent. N Engl J Med 1993;328:438-440

Lerman C, Kash K, Stefanek M, Young women at increased risk of breast cancer: perceived risk, psychological well being and surveillance behavior. Monogr Natl Cancer Inst. 1994;16: 171-176

Lerman C, Lustbader E, Rimer B, Daly M, Miller S, Snads C, Balshem A: Effects of individualized breast cancer risk counseling: a randomized trial. J Natl Cancer Inst 1995, $87: 286-292$ 
Lerman C, Rimer B, Trock B, Balshem A, Engstrom P, 1990; Factors associated with repeat adherence to breast cancer screening. Prev Med 1990; 19:279-90

Lindfors K, Rosenquist C. The cost-effectiveness of mammographic screening strategies. JAMA. 1995;274:881-4

Longnecker M, berlin J, Orza M, Chalmers, A meta-analysis of alcohol consumption in relation to risk of breast cancer, Journal of American Medical Association, 1988; 260: 652-656

Love R, Brown R, Davis J, et al, Frequency and Determinants of Screening for Breast Cancer in Primary Care Group Practice, Archives of Internal Medicine. September 1993

Lucey C, Kumar S, "Assesing mammography rates for Medicaid recipients age 50 to 65 years using claims data from a southeastern state", Journal of Louisiana State Medical Society, 1996, Jul;148(7):303-308

MacMohan B, Cole P, Brown J, Etiology of human breast cancer: a review, Journal of National Cancer Institute, 1973; 50: 21

MacMohan B, Cole P, Lin M, Lowe CR, Mirra AP, Ravnihar B, et al, Age at first birth and breast cancer risk, Bull World Health Organ, 1970; 43: 209-221

Mark D, Hlaty M, Califf R et al., Cost effectiveness of thrombolytic therapy with tissue plasminogen activator as compared with strptokinase for actual myocardial infarction. $\mathrm{N}$ Eng J Med. 1995;332:1418-24

McCance K, Mooney K, Smith K, Field R. Validity and reliability of breast cancer knowledge test. Am J Prev Med 1990; 6:93-98

McCaul K, Branstetter A, Schroeder D, Glasgow R. What is the relationship between breast cancer risk and mamography screening? A meta-analytic review. Health Psychol 1996; 15(6): 423-9

McTiernan A, Gilligan MA, Redmond C. Assessing Individual Risk for Breast Cancer: Risky Business", Journal of Clinical Epidemiology, 1997; 50(5): 547-556

(A) Miller A, Baines C, To T, Wall C. Canadian National Breast Screening Study: 1. Breast cancer detection rate and deaths among women aged 40-49. Can Med Assoc J 1992;147:1459-1476

(B) Miller A, Baines C, To T, Wall C. Canadian National Breast Screening Study: 1. Breast cancer detection rate and deaths among women aged 40-49. Can Med Assoc J 1992;147:1477-1494 
Miller D, Homan S, Determining transition probabilities, Medical Decision Making 1994; 14:52-58

Miller L. Correlations: Description or Inference? Journal of Agricultural Education. 1994; 35(1): 5-7

Murray C, Evans D, Acharya A, Baltussen R. Development of WHO guidelines on generalized cost effectiveness analysis. Health Economics 2000; 9: 235-251

National Cancer Advisory Board Recommendations for women 40-49, Rockville, MD,

National Cancer Institute, Bethesda, MD, 2003 (www.nci.nih.gov)

National Cancer Institute, March 1997

National Cancer Institute, Prevention of Breast Cancer (208/04730) (www.graylab.ac.uk/cancernet/304730.html)

National Institutes of Health Consensus Development Conference Statement Breast cancer screening for women ages 40-49, Bethesda, MD, January 21-23, 1997

National Pharmaceutical Council, Pharmaceutical benefits under state medical assistance programs, 1997: pg. no: A3

National Pharmaceutical Council. Pharmaceutical benefits under State Medical Assistance Programs. National Pharmaceutical Council, Reston, VA. 1999

NIH Consensus Statement. Breast cancer screening for women ages 40-49. J Natl Cancer Inst. 1997;89:1015-20

Noe L, Becker R, Gradishar W, et al., The cost-effectiveness of tamoxifen in the prevention of breast cancer. American Journal of Managed Care 1999; 5(suppl): S389S406

Nystrom L, Rutqvist L, Wall S, Lindgren A, Linqvist M, Ruden S, et al., Breast cancer screening with mammography: overview of Swedish randomized trials. Lancet. 1993; 341: $973-8$

Ottman R, Pike MC, King MC, et al., Practical guide for estimating risk for familial breast cancer, Lancet 1983;2:556-558

Parker J, Gebretsadik T, Sabogal F, Newman J, Lawson HW, "Mammography screening among California Medicare beneficiaries: 1993-1994", American Journal of Preventive Medicine, 1998, Oct;15(3):198-205 
Pauker S, McNeil B, Impact of patient preferences on the selection of therapy, J Chronic Disease 1981;34:77-86

Powles T, Eeles R, Ashley S, Easton D, Chang J, Dowsett M et al., Interim analysis of the incidence of breast cancer in the Royal Marsden Hospital tamoxifen randomized chemoprevention trial, Lancet 1998;352:98-101

Price J, Economically disadvantaged female's perceptions of breast cancer and breast cancer screening. J Natl Med Assoc 1994;86(12):899-906

Primatesta P, Poulter N, Lipid concentrations and the use of lipid lowering drugs: evidence from a national cross sectional survey. BMJ 2000;321:1322-5

Pritchard K, Is tamoxifen effective in prevention of breast cancer, Lancet 1998;351:145167

Pritchard K, Is tamoxifen effective in prevention of breast cancer? The Lancet 1998; 352:80-81

Ransohoff D, Harris R. Lessons from the mammography screening controversy, Ann Intern Med. 1997;127: 1029-1034

Report of the organizing committee and collaborators, Falun meeting. Falun, Sweden, 1996. Breast cancer screening with mammography in women aged $40-49$ years. Int J Cancer 1996;68:693-9

Ries L, Kosary C, Hankey B, Miller B et al., SEER Cancer Statistics Review, 1963-1994: Tables and Graphs. Bethesda, MD: National Cancer Institute; 1997. NIH Publication No. 97-2789

Riley G et al., Medicare payments from diagnosis to death for elderly cancer patients by stage at diagnosis, Medical Care; 33:828-841

Roberts M, Alexander T, Anderson T et al., Edindburgh trial of screening for breast cancer: mortality at seven years. Lancet 1990;335:241-246

Romieu I, Berlin J, Colditz G, Oral contraceptives and breast cancer: review and metaanalysis, Cancer, 1990;66: 2253-2263

Rosenquist C, Lindfors K. Screening mammography beginning at age 40 years, Cancer 1998; 82: 2235-40

Rutledge D, Hartmann W, Kinman P, Winfield A, Explorational of factors affecting mammography behaviors, Preventive Medicine, 1988,17: 412-422 
Salzmann P, Kerlikowske K, Phillips K. Cost effectiveness of extending screening mammography guidelines to include women 40 to 49 years of age, Annals of internal Medicine 1997;127(11):955-965

Saywell R, Champion V, Skinner C et al., Cost effectiveness comparison of five interventions to increase mammography screening. Preventive Medicine 1999; 29: 374382

Scandinavian Simvastatin Survival Group, Randomized trial of cholesterol lowering in 4444 patients with coronary heart disease: the Scandinavian Simvastatin Survival Study (4S). Lancet 1994; 344:1383-9

Seidman $\mathrm{H}$ et al., Survival experience in the breast cancer demonstration project. Cancer J Clin. 1987 Sep-Oct;37(5):258-90

Seltiz C, et al., "Research methods in social relations", 3rd edition, pg no. 309, New York :Holt, Rinehart and Winston.

Serxner S, Chung C, Trend analysis of social and economic indicators of mammography use in Hawaii, American Journal of Preventive Medicine, 1992; 8: 303-308

Shames L et al., Blood levels of organochlorine residues and risk of breast cancer. J Natl Cancer Inst 1994, 85(8):1642-1643

Shapiro S et al., Ten to fourteen year effect of screening on breast cancer mortality. J Natl Cancer Inst 1982;69:349

Shapiro S. The status of breast cancer screening: A quarter of a century of research. World J Surg 1989;13:9-13

Shapiro S, Venet W, Strax P, Venet L. Periodic screening for breast cancer: The Health Insurance Plan Project and Its Sequelae, 1963-1986. Baltimore: Johns Hopkins University Press, 1988

Siegel J, Weinstein M, Russell L, Gold M. Recommendations for reporting cost effectiveness analyses. JAMA 1996;276:1339-1341

Siegel J, Berwick D, Cost-effectiveness and clinical preventive services, Guide to Clinical Preventive Services, Second Edition, Prepared for the U.S. Preventive Services Task Force

Smart C, Hendrick R, Rutledge J, Smith R. Benefit of mammography screening in women age 40-49 years. Cancer 1995;75:1619-26 
Smith T, Hillner B. Tamoxifen should be cost-effective in reducing breast cancer risk in high-risk women. J Clin Oncol 2000;18: 284-286

Smith and Hillner study (http://www.ahcpr.gov/data/94drgb.htm)

Sonnenberg F et al., Markov models in medical decision making: A practical guide, Medical Decision Making 1993;13:322-338

Spiegelman D, Colditz GA, Hunter D, Hertzmark E. Validation of the Gail et al., model for predicting individual breast cancer risk, Journal of the National Cancer Institute, 1994, 86(8): 600-607

Sporn MB, Newton DL. Chemoprevention of cancer with retinoids. Fed Procs $1979 ; 38: 2528-2534$

Standing Medical Advisory Committee. The use of statins. Lon-don: Department of Health, 1997

Stegfanek M, Bilaterla prophylactic mastectomy: Issues and concerns, J Natl Cancer Inst Monogr 1995; 17:37-42

Sweeney F, Newton W, Tamoxifen for prevention of breast cancer in high-risk women, The Journal of Family Practice, 1999; 48(2): 90-91

Tabar L et al., Efficacy of breast cancer screening by age, Cancer 1995;75:2507-17

Tabar L, fagerberg G, Duffy SW, Day NE, Gad A, Grontoft O, Update of two Swedish two-county program of mammographic screening for breast cancer, Raadiol Clin North America, 1992; 30: 187-210

Tabar L,Fagerberg C, Gad A, et al., Reduction in mortality from breast cancer after mass screening with mammography. Lancet 1985;1:829-832

Taplin et al., Journal of National Cancer Institute, 1995: 87(6): 417-426

Taplin S, Thonpson R, Schnitzer F et al., Revisions in the risk based breast cancer screening program at Group Health Cooperative. Cancer 1990; 66: 812 -818

Terenius L. Effect of anti-oestrogens on initiation of mammary cancer in the female rat. Eur J Cancer 1971;7:65-70

The Urban Institute (www.urban.org/news/factsheets/medicaidFS.html)

Thomas D et al., Randomized trial of breast self-examination in Shanghai, J Natl Cancer Inst 1997;89:355-65 
Trueman P, Drummond M, Hutton. Developing guidance for budget impact analysis. Pharmacoeconomics 2001;19(6):609-621

U.S. Bureau of Census. Statistical Abstract of the United States. 11th ed. Washington, DC: U.S. Gov Pr Office; 1991:84

U.S. Census Bureau (Vital Statistics), the Official Statistics, Statistical Abstract of the United States: 1998

U.S. Department of Labor, Bureau of Labor Statistics (http://stats.bls.gov)

UK Trial of Early Detection of Breast Cancer Group. Breast cancer mortality after 10 years in the UK Trial of early Detection of Breast Cancer. Breast 1993;2:13-20

U.S. Department of Health and Human Services, US Preventive Task Force; Guide to Clinical Preventive services, Screening for breast cancer (ed 2), Philadelphia, PA, Williams \& Wilkins, 1996, pp73-87

Veronesi U, Maisonneuve P, Costa A, Sacchini V, Maltoni C, Robertson C. et al., Prevention of breast cancer with tamoxifen, Lancet 1998;352:93-97

Vogel V. High-risk populations as targets for breast cancer prevention trials Prev Med 1991 Jan;20(1):86-100

Vogel V, Bondy M, Halabi S, et al., Validation of Gail's breast cancer risk model, Proc ASCO 1993: 12; 167

Wald N, Chamberlain J, Hackshaw A et al., Consensus conference on breast cancer screening; Report of the evaluation committee. Oncology 1994;51:380-389

Wolff $\mathrm{M}$ et al., Blood levels of organochlorine residues and risk of breast cancer. J Natl Cancer Inst 1993, 85(8):648-652

Woloshin S, Schwartz LM, Black WC, Welch HG. Women's perceptions of breast cancer risk: how you ask matters. Med Decis Making 1999;19(3):221-9

Wright C, Breast cancer screening: A different look at the evidence, Surgery 1986;594597

www.hcfa.gov/medicaid/mservice.html

Zapka J, D Hosmer, M Costanza, et al, Changes in Mammography Use: Economic, Need and Service Factors, American Journal of Public Health. 1992. 82(10)

Zapka J, Stoddard A, Costanza M, Greene H, Breast cancer screening by mammography: utilization and associated factors. Am J Public Health 1989; 79:1499-1502 
Zethraeus N, Lindgren, Johnell O, Jonsson B, A computer model to analyze the costeffectiveness of hormone replacement therapy-a revised version, SSE/EFI Working Paper Series in Economics and Finance, No. 368, Stockholm School of Economics, March 2000 
APPENDIX A

COVER LeTTERS \& QUeSTIONNAIRES 


\title{
Introductory Cover Letter
}

\author{
August 5, 2002
}

Dear Madam:

Breast cancer is an important health problem. It is one of the major causes of deaths in West Virginia. We at the Medicaid Program are working with the West Virginia University School of Pharmacy to deal with this important concern.

You may receive a survey on breast cancer screening and its risk assessment in the next few weeks. Your participation in this survey is entirely up to you and will not affect your services in any way. However, the information that you may provide by answering the survey will help us to understand the health care needs of West Virginia women as they relate to breast cancer. Hence, your participation in this study will be very much valued. Thank you.

Sincerely,

Nancy V Atkins, MSN, RNC, NP

Commissioner 


\section{First Cover Letter}

August 8, 2002

\section{Dear Madam:}

Breast cancer is an important health problem. It is one of the major causes of deaths in West Virginia. We at the Medicaid Program are working with the West Virginia University School of Pharmacy to deal with this important concern. This study is part of a doctoral (Ph.D.) research project.

Please find attached a survey on breast cancer screening and its risk assessment. We would really appreciate it if you will kindly take a few minutes to complete the survey and return it in the postage-paid business reply envelope.

Your participation in this survey is entirely up to you and will not affect your Medicaid services in any way. Your responses will be coded, and your name will not appear in any reports. Your names will be stored in the master codebook by the investigator. This codebook will be destroyed upon completion of this study. Therefore, we assure you of as much confidentiality as legally possible.

Although we hope that you will answer all of the questions, you do not have to answer any question that makes you uncomfortable. However, your response will provide useful information and is very important to the results of this study. Hence, your participation in this study will be very much valued. Thank you.

If you have any questions or concerns, please contact Dr. Suresh Madhavan (304 293-1652) at the West Virginia University School of Pharmacy. Thank you.

Sincerely,

Nancy V Atkins, MSN, RNC, NP

Commissioner 


\section{Second Cover Letter}

August 22, 2002

\section{Dear Madam:}

About two weeks ago, we sent you a survey on breast cancer screening and its risk assessment. If you have already completed the survey and returned it we thank you for your time and participation. If you have not completed the survey we request you to kindly do so.

We understand that you are busy or may not have received the survey. However, your views are extremely important to us. Therefore, we are again sending you this survey and would appreciate it if you will kindly complete the survey and return it in the postage-paid business reply envelope.

Your participation in this survey is entirely up to you and will not affect your Medicaid services in any way. Your responses will be coded, and your name will not appear in any reports. Your names will be stored in the master codebook by the investigator. This codebook will be destroyed upon completion of this study. Therefore, we assure you of as much confidentiality as legally possible.

Although we hope that you will answer all of the questions, you do not have to answer any question that makes you uncomfortable. However, your response will provide useful information and is very important to the results of this study. Hence, your participation in this study will be very much valued. Thank you.

If you have any questions or concerns, please do not hesitate to contact Dr. Suresh Madhavan (304 293-1652) at the West Virginia University School of Pharmacy. Thank you.

Sincerely,

Nancy V Atkins, MSN, RNC, NP

Commissioner 


\section{Third Cover Letter}

September 5, 2002

Dear Madam:

About four weeks ago, we sent you a survey on breast cancer screening and its risk assessment. If you have already completed the survey and returned it we thank you for your time and participation. If you have not completed the survey we request you to kindly do so.

We understand that you are busy or may not have received the survey. However, your views are extremely important to us. Therefore, we are again sending you this survey and would appreciate it if you will kindly complete the survey and return it in the postage-paid business reply envelope.

Your participation in this survey is entirely up to you and will not affect your Medicaid services in any way. Your responses will be coded, and your name will not appear in any reports. Your names will be stored in the master codebook by the investigator. This codebook will be destroyed upon completion of this study. Therefore, we assure you of as much confidentiality as legally possible.

Although we hope that you will answer all of the questions, you do not have to answer any question that makes you uncomfortable. However, your response will provide useful information and is very important to the results of this study. Hence, your participation in this study will be very much valued. Thank you.

If you have any questions or concerns, please do not hesitate to contact Dr. Suresh Madhavan (304 293-1652) at the West Virginia University School of Pharmacy. Thank you.

Sincerely,

Nancy V Atkins, MSN, RNC, NP

Commissioner 


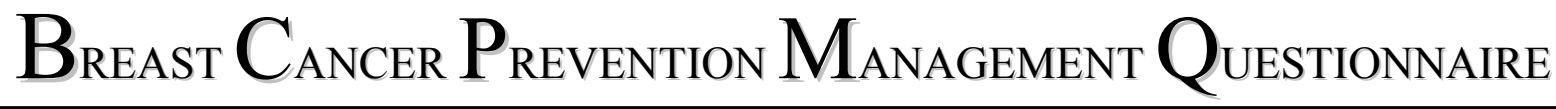

Please answer the following questions to the best of your ability by marking $(X)$ in the box that represents the best chosen answer.

Q1. Have you EVER had a mammogram? (A mammogram is an x-ray of the breast to look for breast cancer.)
Yes
No
Not sure

If your answer to Q1 is "Yes", then continue with Q2. If your answer to Q1 is 'no' or 'not sure', then go to Q5.

Q2. How long has it been since you had your LAST mammogram?

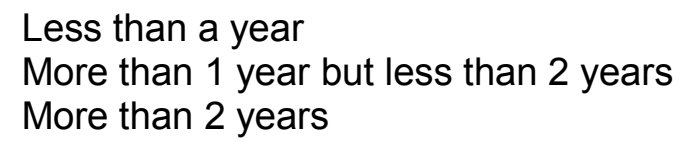

Q3. Why was your LAST mammogram done?
Routine checkup
Follow-up for a lump detected
Breast problem other than breast cancer
Follow up to breast cancer diagnosed in the past

Q4. What was the result of your last mammogram?
Normal
Abnormal
Do Not Know

Q5. Have you EVER taken the drug tamoxifen at the recommendation of your doctor?

[This drug is used in treatment of breast cancer and also to lower the risk of developing breast cancer in women who are healthy but at relatively high risk of developing breast cancer as determined by a physician]
Yes
No
Not sure

Q6. In your opinion, how do you compare your likelihood of developing breast cancer in next 5 years to that of any woman in the general population?
Lower
Similar
Higher
Not sure

Q7. In your opinion, how do you compare your lifetime risk of developing breast cancer to that of any woman in the general population?
Lower
Similar
Higher
Not sure

Q8. Have you ever had a breast biopsy?

("Breast biopsy" refers to surgical removal of sample of breast tissue, usually under local or general anesthesia, for the purpose of determining the presence or absence of cancer.)
Yes
No
Do Not Know 
If your answer to Q8 is "Yes", then continue with Q9. If your answer to Q8 is 'no', then go to Q12.

Q9. How many breast biopsies have you had in your lifetime?

Q10. Did your doctor ever tell you that one of your biopsies showed atypical hyperplasia (abnormal growth of cells)?
Yes
No
Do Not Know

Q11. Have you ever had a breast biopsy that showed lobular carcinoma in situ (LCIS) or ductal carcinoma in situ (DCIS)? (These are non-invasive breast cancers involving only the cells lining the milk ducts in the breast with no evidence that the disease has spread outside of these ducts.)
Yes
No
Do Not Know

Q12. Have you ever had breast cancer?

Yes $\quad \square$ No

Q13. What is your age?

(years)

Q14. How old were you when you had your first menstrual cycle?
Less than 12 years old
12 years or greater
Do Not Know

Q15. Have you ever given birth?

Yes $\quad \square$ No

If your answer to Q15 is "Yes", then continue with Q16. If your answer to Q15 is 'no', then go to Q17.

Q16. How old were you when your first child was born?
Less than 30 years old
30 years old
More than 30 years old
Don't know

Q17. Did your mother have breast cancer?
Yes
No
Do Not Know

Q18. Do you have or have you had any sister(s)?

$\square$ Yes $\quad \square$ No

If your answer to Q18 is "Yes", then continue with Q19. If your answer to Q18 is 'no', then go to Q20.

Q19. Do any of your sisters or did any of your sisters have breast cancer?

Yes (If yes, how many of your sisters had breast cancer?

No 
Q20. Do you have or have you had any daughter(s)?

$\square$ Yes $\quad \square$ No

If your answer to Q20 is "Yes", then continue with Q21. If your answer to Q20 is 'no', then go to Q22.

Q21. Do any of your daughters or did any of your daughters have breast cancer?

Yes (If yes, how many of your daughters had breast cancer?

No

Q22. Please indicate the extent of agreement or disagreement with each of the following statements:

The risk of breast cancer increases with age

Women with close relatives with breast

cancer have higher risk of breast cancer

Breast cancer is usually painful when it

is just getting started

Giving birth to a first child after 30 years

of age increases the risk of breast cancer

A woman currently using birth control pills has a slightly greater risk of breast cancer as compared to a woman not using them

Agree

Long term use of estrogen replacement therapy

(also known as hormone replacement therapy)

after menopause, slightly increases breast cancer risk

Agree

Agree

$\square$ Agree
$\square$ Agree

$\square$ Agree
$\square$ Agree

Mammograms can detect breast lumps early

Regular mammography screening reduces

the risk of dying from breast cancer

Mammography can spread breast cancer

Mammography can cure breast cancer

One mammogram is enough to ensure

that you will not get breast cancer

Breast cancers can be prevented

Alcohol consumption increases the risk of

Obesity (being overweight) is a risk factor

for breast cancer

Agree

Agree

Agree

Agree

Agree
Agree

Agree

Agree

Agree

Don't Agree

Don't know

Don't know

Don't know

Don't know

Don't Agree

Don't know $\square$ Don't Agree

Don't know

Don't Agree

Don't know

Don't Agree

Don't know

Don't Agree

Don't know

Don't Agree

Don't know 
Q23. Are you currently a West Virginia Medicaid enrollee?
Yes
No
Don't know

Q24. Were you a West Virginia Medicaid enrollee at any time in the year 2001 ?
Yes
No
Don't know

Q25. A large clinical trial in women with a relatively high risk of developing breast cancer showed that taking the drug tamoxifen for a period of 5 years cuts the risk of developing breast cancer by almost one half for that 5-year time period during which the drug was being taken.

We would like to know how willing you are to take this drug if your health insurance program pays for it and your doctor tells you that you are at a relatively high risk of developing breast cancer.

However, before you answer this question please consider the following benefits and harmful effects that may result from consuming the drug tamoxifen:

BENEFITS of Tamoxifen - Taking the drug tamoxifen will reduce your risk of breast cancer by almost one-half.

HARMFUL EFFECTS of Tamoxifen - Taking the drug tamoxifen will increase the risk of other events such as endometrial cancer (cancer of uterus lining) by 2.5 times, pulmonary embolism (blood clot formation in lungs) by 3 times and cataract (thickening of cornea) slightly.

However, it has to be noted that in general a woman is much more likely to develop breast cancer than endometrial cancer.

Bearing in mind these benefits and risks of tamoxifen (and also if your insurance pays for it and you are told by your doctor that you are at high risk for breast cancer), will you be willing to take the drug tamoxifen for reducing your risk of developing breast cancer?

Yes $\quad \square$ No $\quad \square$ Not sure

Thank you for taking the time to complete this questionnaire.

Disclaimer: This study uses Gail Model Risk Assessment Tool (as modified by the National Surgical Adjuvant Breast and Bowel Project (NSABP) for the Breast Cancer Prevention Trial) to estimate a woman's risk of developing invasive breast cancer. The survey is designed for research purposes only, and is not intended for diagnosing or treating a health problem or a disease. If you have concerns about your own risk of developing breast cancer, you should consult your health care provider. 


\section{Breast Cancer Prevention Management Questionnaire: Non-Response Survey}

Dear Madam:

We sent you a survey asking you about your general awareness regarding breast cancer and certain factors that may affect your chances of developing breast cancer. Since your views are extremely important to us, we would like to know your reason for not responding to this survey and some key information for the study. Please answer the few questions below and mail it to us in the postage-paid business reply envelope. If you have any questions or concerns, please do not hesitate to contact Dr. Suresh Madhavan (304 293-1652) at the West Virginia University School of Pharmacy. Thank you.

Sincerely,

Nancy Atkins, MSN, RNC, NP

Commissioner

I did not respond to the survey because:

$\square$ I did not receive it

$\square$ I do not respond to mail surveys

$\square$ I was not at home

$\square$ I did not have time to complete it

$\square$ Lost the survey

$\square$ The survey was too long

$\square$ The survey was confusing

$\square$ I haven't finished it yet

$\square$ I am not interested in this issue

$\square$ Other reasons (Please specify):

1. Have you ever had breast cancer?

$\square$ Yes

$\square$ No

2. Have you ever had a breast biopsy that showed lobular carcinoma in situ (LCIS) or ductal carcinoma in situ?
$\square$ Yes
$\square$ No
Don't know

3. How old are you? (years)

4. How old were you when you had your first menstrual period?

$\square$ Less than 12 years old $\square 12$ years old $\quad$ More than 12 years old $\square$ Don't know

5. How old were you when your first child was born?
$\square$ Less than 30 years old
30 years old $\square$ More than 30 years old
$\square$ Don't know
$\square$ No children

6. Did your mother have breast cancer? $\square$ Yes $\square$ No

7. Did your sisters (if any) have had breast cancer? If yes how many sisters? (if none, enter ' 0 ' in the blank below)
$\square$ Yes
$\square$ No
Number of sisters who have had breast cancer

8. Did your daughters (if any) have had breast cancer? If yes how many daughters? (if none, enter ' 0 ' in the blank below)

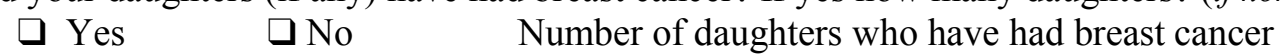

9. How many breast biopsies have you had in your lifetime? [If none, indicate ' 0 ' in the blank below] (number of biopsies)

10. Did your doctor ever tell you that one of your biopsies showed atypical hyperplasia?
$\square$ Yes
$\square$ No
$\square$ Not sure

11. What is your race?

$\square$ Caucasians $\square$ African Americans
$\square$ Others(please specify)

a Pacific/Asian Islanders 


\section{RESUME}

\section{Rohit D. Borker}

\section{EDUCATION}

WEST VIRGINIA UNIVERSITY

Doctoral Candidate, Pharmaceutical Systems \& Policy

08/97-05/03

Ph.D. in Pharmaceutical Sciences

Major: Health Outcomes Research

Cumulative GPA: 3.93/4.00

UNIVERSITY OF PUNE, INDIA

Bachelor of Science, Pharmacy

Cumulative Grade: Distinction (72.41\%)

06/92-06/96

\section{RESEARCH EXPERIENCE}

Schering-Plough Health Outcomes Research Fellow

09/00-09/01

- Epidemiological and health-outcomes based review of disease conditions

Research Assistant

$06 / 98-12 / 00$

Pharmacy Immunization Project (1995-2000), funded by grant (U66/CCU312177)

from Centers for Disease Control and Prevention (CDC), West Virginia University

- Developed surveys and performed data analysis

- Developed manuscripts and presentations

- Supervised research staff and coordinated research activities

Ph.D. Dissertation Project

01/99-05/03

Title: "Economics of Breast Cancer Preventive Strategies in a Medicaid Program"

- Developed conceptual framework and methodology

- Developed the Multi-state Markov Process to determine incremental life-expectancy and cost of chemopreventive tamoxifen and mammography screening

\section{PROFESSIONAL EXPERIENCE}

GlaxoSmithKline, Global Health Outcomes, RTP, NC

12/01-Present

Therapeutic Area: Respiratory

- Involved with decision and budget impact modeling

- Involved with secondary database analysis

- Providing health outcomes input in clinical trials

Schering-Plough Corporation, Dept. of Health Economics,

05/00-08/00

New Jersey, USA (Summer Intern)

- Constructed an excel-based cost-minimization model and developed a Visual Basic

- Application-based interface for an oncology drug

- Proposed and developed a validation plan for a cost-effectiveness model

- Assisted in developing an interface for a cost-effective model for a rheumatoid arthritis drug

Hoechst Marion Roussel (HMR) (now Aventis) Ltd., India (1 year trainee)

08/96-07/97

- Received training in manufacturing and quality control 


\section{RELEVANT COURSES}

Health Outcomes: $\quad$ Pharmacoeconomics, Outcomes Research, Health Economics, Epidemiology, Micro Economics, Econometrics, Computational Methods, Project Evaluation

Research Methods: $\quad$ Secondary Data Analysis, Multivariate Analysis, Survey Research Methods, Data Analysis using SPSS and SAS

Health Administration: Healthcare Organization \& Operations, Managed Care, Health Systems

Marketing: Health Services Marketing, Consumer Behavior

\section{COMPUTER PROFICIENCY}

- Worked with DATA TreeAge, Visual Basic Application, @ RISK (Monte Carlo Simulation), SPSS, SAS mainframe, EViews (Econometrics), MS-Word, MS-Excel, and MS-Power Point

- Worked with BRFSS and currently working with West Virginia Medicaid data

\section{PUBLICATIONS}

M.Halpern, R.Stanford, R.Borker. The burden of COPD in the U.S.A.: results from the Confronting COPD survey. Respiratory Medicine Vol. 97 (2003) (SUPPLEMENT C). S81

Madhavan S, Rosenbluth S, Amonkar M, Fernandes A, Borker R. Immunization predictors in rural adults under 65 years of age. Journal of Health Care for the Poor and Underserved. 2003; 14 (1): 100-21

Sheth K, Borker R, Emmett A, Rickard K, Dorinsky P. The cost-efficacy comparison of fluticasone propionate plus salmeterol versus montelukast in the treatment of adults with persistent asthma. Pharmacoeconomics 200220 (13) Page 909-918

Madhavan S, Rosenbluth S, Amonkar M, Borker R, and Richards T. Pharmacists and Immunizations: A National Survey. Journal of American Pharmaceutical Association 2001; 41:32-45

Rosenbluth S, Madhavan S, Borker R, Maine L. Pharmacy Immunization Partnerships: A Rural Model" Journal of American Pharmaceutical Association. 2001; 41:100-7

Madhavan S, Borker R, Fernandes A, Amonkar M, Rosenbluth A. Assessing Predictors of influenza and pneumonia vaccination in rural senior adults. Excepted in Journal of Health and Social Policy.

\section{PODIUM PRESENTATION}

Borker R, Stanford S, Reisner C, Fischer T, Morris A, Zhu J, Barnhart F. Direct cost savings associated with the use of cilomilast in patients with chronic obstructive pulmonary disease, CHEST 2002, the $68^{\text {th }}$ Annual International Meeting, San Deigo, CA, November 2002

Borker R., Madhavan S., Amonkar M., Rosenbluth S., "Pharmacists \& Immunization: A West Virginia Pharmacists Survey", ESAS Section, $148^{\text {th }}$ Annual Meeting of the American Pharmaceutical Association, San Francisco, March 2001

\section{SELECTED POSTER PRESENTATIONS}

Rascati K, Stanford R, Borker R Comparison of risk for hospital and/or emergency department visits for Medicaid patients with COPD. ISPOR 8th Annual International Meeting, May 18-21,2003

Rascati K, Stanford R, Borker R. Comparison of Hospitalization Risk for Medicaid Patients with Chronic Obstructive Pulmonary Disease by Medication Regimen, ATS International Conference in Seattle, Washington, 2003 
Borker R, Stanford R, Emmett A, Dorinsky P, Rickard K. Cost-efficacy of fluticasone propionate plus salmeterol versus montelukast in patients with persistent asthma uncontrolled on short acting beta -agonist $^{-}$ alone, American College of Allergy, Asthma, and Immunology $60^{\text {th }}$ Annual Meeting, San Antonio, TX, November 2002

RH Stanford, R Borker, PM Dorinsky, PJ Pepsin, CJ Kalberg, AH Emmett, KA Rickard. The costs and efficacy of fluticasone propionate/salmeterol combination versus montelukast in the treatment of adults with persistent asthma, CHEST 2002, the $68^{\text {th }}$ Annual International Meeting, San Deigo, CA, November 2002 ,

Borker R., Madhavan S., Simon K., Higa G., Amonkar A., Scott V., "Long-term benefits of chemopreventive tamoxifen and routine mammography screening in women who are at increased risk of breast cancer", International Society for Pharmacoeconomic and Outcomes Research (ISPOR), $7^{\text {th }}$ Annual International Meeting, May 2002 (This poster received the 'Best Student Poster Presentation' award at the ISPOR meeting)

Madhavan S., Rosenbluth S., Amonkar M., Borker R., Richards T., "Identifying Immunization Rates, Barriers, and Predictors of Immunization in Elderly (over 65 years of age)", $34^{\text {th }}$ National Immunization Conference, Washington D.C., July, 2000

Madhavan S., Rosenbluth S., Amonkar M., Borker R., Richards T., Scott V., Maine L., "Identifying Predictors of Adult Immunization", 34 ${ }^{\text {th }}$ National Immunization Conference, Washington D.C., July, 2000

Madhavan S., Rosenbluth S., Amonkar M., Borker R., Richards T., Nau D., "Identifying Barriers of Adult Immunization", $34^{\text {th }}$ National Immunization Conference, Washington D.C., July, 2000

Borker R., Madhavan S., Amonkar M., Higa G., Simon K., "Economics of Primary Prevention of Breast Cancer in WV Medicaid Population", ESAS Section, $147^{\text {th }}$ Annual Meeting of the American Pharmaceutical Association, Washington, D.C., March 2000

Madhavan S., Rosenbluth S., Amonkar M., Borker R., Nau D., Scott G., and Richards T., "Pharmacists' Willingness to Provide Immunization Services: A National Survey", $33^{\text {rd }}$ National Immunization Conference, Dallas, TX, June 20-25, 1999

Madhavan S., Rosenbluth S., Amonkar M., Borker R., Nau D., Scott G., and Richards T., "Enhancing Immunization roles of Rural Community Pharmacists by Building Partnerships", Proceedings of the WVU School of Medicine Research day", Morgantown, March 19, 1999

Madhavan S., Rosenbluth S., Amonkar M., Borker R., Nau D., Scott G., and Richards T., "Does Immunization Provider Site Influence Completion Rates and Perceptions of Quality and Satisfaction with Immunization Services?" $32^{\text {nd }}$ National Immunization Conference, Atlanta, Georgia, July 21-24, 1998

\section{HONORS/AFFILIATIONS}

Recipient of the 'Best Student Poster Presentation' award at the ISPOR, $7^{\text {th }}$ Annual International Meeting Recipient of Graduate Research Award, School of Pharmacy Recipient of Graduate Teaching Award, School of Pharmacy Member of Rho Chi Honor Society Graduate Council Representative (School of Pharmacy) for West Virginia University Member of International Society for Pharmacoeconomics \& Outcomes Research (ISPOR) Member of American Pharmaceutical Association (APhA) 